\title{
Energy Efficient Visible Light Communications Relying on Amorphous Cells
}

\author{
Rong Zhang, Member, IEEE, Holger Claussen, Senior Member, IEEE, Harald Haas, Member, IEEE, \\ and Lajos Hanzo, Fellow, IEEE
}

\begin{abstract}
In this paper, we design an energy efficient indoor visible light communications (VLC) system from a radically new perspective based on an amorphous user-to-network association structure. Explicitly, this intriguing problem is approached from three inter-linked perspectives, considering the cell formation, link-level transmission and system-level optimisation, critically appraising the related optical constraints. To elaborate, apart from proposing hitherto unexplored amorphous cells (A-Cells), we employ a powerful amalgam of asymmetrically clipped optical orthogonal frequency division multiplexing (ACO-OFDM) and transmitter pre-coding aided multi-input single-output (MISO) transmission. As far as the overall system-level optimisation is concerned, we propose a low-complexity solution dispensing with the classic Dinkelbach's algorithmic structure. Our numerical study compares a range of different cell formation strategies and investigates diverse design aspects of the proposed A-Cells. Specifically, our results show that the A-Cells proposed are capable of achieving a much higher energy efficiency per user compared to that of the conventional cell formation for a range of practical field of views (FoVs) angles.
\end{abstract}

Index Terms-Energy Efficiency, Optical Wireless, Small Cells, Optical OFDM, Optical MIMO.

\section{INTRODUCTION}

1) Background: Improving the attainable energy efficiency has been one of the salient design objectives of modern wireless communications [1]. In the post-4G era, quantifying energy efficiency became a challenge owing to the emerging Heterogeneous Networks (HetNet) in pursuit of 'green' designs [2]-[6]. With the launch of the global 5G research initiatives, the community expanded its horizon from Radio Frequency (RF) cellular networks both to millimetre wave [7] and to optical wireless concepts [8]. Owing to this paradigm-shift to higher frequencies, the disruptive large-scale Multi-Input Multi-Output (MIMO) architecture has attracted substantial interests, with the goal of further improving the achievable energy efficiency [9]. Apart from the classic perspectives on

Manuscript received April 15, 2015; revised September 7, 2015; accepted December 11, 2015. This work was supported in part by the EPSRC project (EP/N004558/1) and in part by the EU under the Concerto project as well as that of the European Research Council's (ERC) Advanced Fellow Grant. The data from the paper can be obtained from the University of Southampton ePrints research repository: $10.5258 / \mathrm{SOTON} / 385179$.

R. Zhang and L. Hanzo are with the Southampton Wireless, School of Electronics and Computer Science, University of Southampton, Southampton SO17 1BJ, U.K. (e-mail: rz@ecs.soton.ac.uk).

H. Claussen is with the Small Cells Research, Bell Laboratories, AlcatelLucent, Dublin 15, Ireland.

H. Haas is with the Li-Fi R\&D Centre, Institute for Digital Communications, University of Edinburgh, Edinburgh EH9 3JL, U.K.

Color versions of one or more of the figures in this paper are available online at http://ieeexplore.ieee.org.

Digital Object Identifier 10.1109/JSAC.2016.2544598 energy efficiency, the novel concepts of wirelessly powered communications [10], [11] are also emerging. Despite all the above advances, there is a paucity of literature on designing energy efficient optical wireless systems, especially indoor Visible Light Communications (VLC) systems.

With the advent of high-power Light Emitting Diodes (LEDs) and high-sensitivity Photo-Diodes (PD), the VLC concept appears to be especially promising in the small-cell family of the $5 \mathrm{G}$ era [12]. By modulating the visible light produced by the LEDs way above the human eye's fusion frequency, the dual goal of communication and illumination can be realised simultaneously. The pioneering implementation of VLC using LEDs was carried out by the Nakagawa laboratory in 2004 [13], which stimulated significant research attention. The link-level data rates of 100s of MBits/s have been reported using state-of-the-art LEDs and photo-detectors [14]. The modulation schemes have evolved from simple pulse based modulation to more sophisticated Asymmetrically Clipped/DC-biased Optical Orthogonal Frequency Division Multiplexing (ACO/DCO-OFDM) [15], [16]. More ambitious GBits/s targets have also been achieved with the aid of optical MIMO techniques [17] and by using advanced LEDs [18]. Apart from these exciting link-level achievements, the systemlevel study of VLC has also been developed for broadening its scope beyond point-to-point applications [19]-[24].

2) Motivation: However, most of the above-mentioned VLC research aimed for increasing the attainable throughput, whilst paying less attention to energy efficiency. In fact, LEDs are primarily used for illumination, where typically a constant DC power is provided to satisfy the illumination requirements and to maintain sufficient forward biasing voltages across the LEDs for communications. Hence, the additional communication function should not perturb the illumination requirements nor should it violate the LEDs physical limits. Desirably, the extra communication-related power consumption invested should also be as low as possible, while maintaining a minimum required Quality of Service (QoS). This is also true when no illumination is required during daytime. Hence, valuable research has been dedicated to link-level energy efficiency focusing on brightness and dimming control with the aid of both modulation-related [25], [26] and coding-related [27], [28] techniques. However, there is no system-level investigation on energy efficient VLC systems supporting multi-users, which may require a radically new design approach.

When considering the attainable system-level energy efficiency of a particular network, the specific structure of associating the users with the network plays a crucial role. As a result, it has limited benefits to optimise the power consumption of
40 
an inefficient user-to-network association structure. Thanks to the flexibility in indoor VLC systems design [12], we advocate an amorphous user-to-network association structure for indoor VLC systems. To elaborate, in conventional structures, the cells are typically formed from a network-centric perspective, without taking into account the users' positions, where the design flow is based on defining a cell constituted by one or more Access Points (APs) and then associating the users with it. In RF cellular systems having hundreds or thousands of random uniformly distributed users, the conventional structure may indeed be applicable. However, when considering an indoor VLC system supporting only a few dozen users, naively applying the conventional structure may become inefficient, since the users are sporadic. Moreover, in VLC systems, the number of APs may be higher than the number of users, creating an ultra dense AP deployment. Hence, we propose Amorphous Cells (A-Cells) from a user-centric point of view by considering the users' positions, where the design flow is based on grouping the users together and then associating the APs with them, resulting in irregular shape cells.

3) Scope: We design an energy efficient indoor VLC system relying on an amorphous structure under practical optical constraints by considering three interlinked design aspects, namely the cell formation, the link-level transmission and the systemlevel power allocation. Logically, the cell formation strategy determines the specific association between the APs and users, while the transmission strategy and the power allocation jointly determine the signal strength and the amount of interference. Explicitly,

- we propose two A-Cells formation techniques, namely the edge-distance and centroid-distance based A-Cells. The beneficial construction of A-Cells constitutes the basis of a structurally energy efficient indoor VLC system;

- we propose a new link-level transmission scheme by amalgamating the ACO-OFDM and our Vector Transmission (VT) and Combined Transmission (CT) based Multiple Input Single Output (MISO) transmission of [12];

- we propose an efficient low-complexity algorithm for maximising the system-level energy efficiency employing the advocated link-level transmission scheme associated with the proposed A-Cells.

Our paper is organised as follows. In Section II-A, we embark on designing the proposed amorphous structure, while in Section II-B, we discuss the transmission schemes employed and finally we perform system optimisation in Section II-C. The achievable performance of our design is characterised in Section III and we close in Section IV.

\section{SySTEM DESCRIPTION}

Consider an indoor VLC environment having $N$ APs uniformly installed on the ceiling, where each AP is constituted by an array of $L$ LEDs pointing vertically downwards. These APs will be used for communicating with $K$ users and at the same time for providing illumination. As discussed before, supporting wireless communications should not violate the main illumination requirements and should obey the LEDs physical limits. Hence, the communications-related power investment 144 should be as low as possible in order to minimise the perturba- 145 tions imposed on the lightening function, while maintaining a 146 minimum required QoS. This leads to communications-related 147 energy efficiency maximisation as

$$
\max _{\mathcal{F}, \mathcal{T}, \mathcal{P}} f_{t}(\mathcal{F}, \mathcal{T}, \mathcal{P}) / f_{p}(\mathcal{F}, \mathcal{T}, \mathcal{P}),
$$

where $f_{t}(\cdot)$ represents the achievable throughput, which is a 149 function of the cell formation strategy $\mathcal{F}$, of the link-level trans- 150Q1 mission strategy $\mathcal{T}$ and of the power allocation strategy $\mathcal{P}$. 151 Furthermore, $f_{p}(\cdot)$ represents the power consumption, which 152 is also a function of $\mathcal{F}, \mathcal{T}$ and $\mathcal{P}$. Since $\mathcal{F}$ and $\mathcal{T}$ are potentially 153 enumerable, we can reformulate $(1)$ given $\mathcal{F}$ and $\mathcal{T}$ as ${ }^{1}$.

$$
\max _{\mathcal{P}} f_{t \mid \mathcal{F}, \mathcal{T}}(\mathcal{P}) / f_{p \mid \mathcal{F}, \mathcal{T}}(\mathcal{P})
$$

To adopt a clear structure, we will elaborate 1) on the cell 155 formation strategy in Section II-A by describing the channel 156 characteristics in Section II-A1, then introducing the moti- 157 vation of A-Cells in Section II-A2 and finally detailing the 158 construction of A-Cells in Section II-A3; 2) on the transmission 159 strategy in Section II-B by introducing ACO-OFDM and MISO 160 transmission in Section II-B1 and Section II-B2 respectively, 161 and then introducing the optical constraints in Section II-B3; 162 3 ) on the power allocation strategy in Section II-C by out- 163 lining our problem formulation, transformation and simpli- 164 fication in Section II-C1, Section II-C2 and Section II-C3, 165 respectively.

\section{A. Amorphous Structure}

Fixing $\mathcal{F}$ in (1) not only reduces the complexity of the prob- 168 lem, but also constitutes a logically appealing arrangement, 169 since cell formation is the pivotal system design stage.

1) Channel Characteristics: Before introducing the cell 171 formation strategy, a brief description of the VLC channel char- 172 acteristics is essential. The optical channel between the $k$ th user 173 and the $n$th AP is constituted by both the direct Line-of-Sight 174 (LoS) component $h_{k, n}^{0}$ and its reflections, but we only consider 175 the first reflection $h_{k, n}^{1}$, since higher-order indirect reflections 176 are typically negligible. Specifically, the LoS component is 177 given by [13]

$$
h_{k, n}^{0}=\frac{\left(m_{L}+1\right) A_{P D}}{2 \pi d^{2}} \cos ^{m_{L}}(\theta) \cos (\psi) f_{o f}(\psi) f_{o c}(\psi),
$$

where the Lambert index $m_{L}=-1 / \log _{2}\left[\cos \left(\phi_{1 / 2}\right)\right]$ depends on the semi-angle $\phi_{1 / 2}$ at half-illumination of the source. $A_{P D}$ is the physical area of the PD receiver, $d$ is the distance between the $k$ th user and the $n$th AP, $\theta$ is the angle of irradiance from the $n$th AP and $\psi$ is the angle of incidence at the $k$ th user. Still referring to $(3), f_{o f}(\psi)$ and $f_{o c}(\psi)$ denote the gain of the optical

\footnotetext{
${ }^{1}$ Re-evaluating (2) upon the change of cell formation and transmission strategy would impose an excessive complexity. Fortunately, indoor VLC systems typically have low-mobility. Hence, updating (2) semi-adaptively, not instantaneously, strikes a comprise, although the optimal updating frequency is application-specific. However, these interesting points are out of our scope.
} 
185 filter and of the optical concentrator employed, respectively. Furthermore, $f_{o c}(\psi)$ can be written as

$$
f_{o c}(\psi)=n_{r}^{2} / \sin ^{2}(\psi), \psi \leq \psi_{F} ; \quad f_{o c}(\psi)=0, \psi>\psi_{F},
$$

where $\psi_{F}$ represents half of the receiver's Field-of-View (FoV) and $n_{r}$ is the refractive index of a lens at a PD receiver. By contrast, the first reflected component is given by [13]

$$
h_{k, n}^{1}=\sum_{v} \sum_{\tau} \frac{\rho_{r} A_{r} d^{2}}{d_{v, \tau, 1}^{2} d_{v, \tau, 2}^{2}} \cos \left(\alpha_{v, \tau}\right) \cos \left(\beta_{v, \tau}\right) h_{k, n}^{0},
$$

where $d_{v, \tau, 1}$ is the distance between the $n$th AP and the $(v, \tau)$ th reflection point, while $d_{v, \tau, 2}$ is the distance between the $(v, \tau)$ th reflection point and the $k$ th user. Furthermore, $\alpha_{v, \tau}$ and $\beta_{v, \tau}$ denote the angle of incidence for the incoming light and the angle of irradiance for the outgoing light at the $(v, \tau)$ th reflection point, having a tiny area of $A_{r}$ and a reflectance factor of $\rho_{r}$. Furthermore, the pair of summations in (5) represent all the reflections from the walls. Finally, the aggregated channel between the $k$ th user and the $n$th AP is given by $h_{k, n}=$ $h_{k, n}^{0}+h_{k, n}^{1}$, where we assumed single-tap channel response. In the following, we use only the LoS component for constructing the A-Cells, but we will use the aggregated channel for the rest of our design.

2) Motivation of A-Cells: Fig. 1 portrays the conventional structure (left) and the amorphous structure (right) for a $15 \mathrm{~m} \times$ $15 \mathrm{~m}$ indoor VLC system having $8 \times 8$ APs (marked by squares) and 20 users (marked by circles) under three typical scenarios (same, more and less number of cells), where the users' positions are drawn from a uniform random distribution, whilst employing the parameters of Table I. Owing to spacelimitations, in Fig. 1 we only show the edge-distance based A-Cells having a predefined distance threshold of $d_{0}=3.5 \mathrm{~m}$.

Conventional cells typically have a fixed shape. For example, we may partition the $15 \mathrm{~m} \times 15 \mathrm{~m}$ indoor environment into four square-shaped cells having $(4 \times 4)=16$ APs per cell, where the users are associated with cells depending on the users' positions relative to the square-shaped boundary amongst the cells. Within each cell, we may switch off the communications function of the specific APs having no LoS links to the users in their vicinity (indicated by hollow small squares), since improving the energy efficiency is our goal. The related examples may be seen in the left of Fig. 1, where we refer to this bench-marker as 'S16'. Similarly, we also have the special bench-marker of 'S1', which represents the scenario of using each AP to create an individual cell and again, the idle mode is used for those APs, which have no LoS links to the users in order to save energy [29].

A common observation concerning the conventional arrangement 'S16' in the left of Fig. 1 is that the resultant cells are all constrained within the four partitioned areas. However, this arrangement may not be the most appropriate. For example, in the southwest cell of Fig. 1a, the 'boundary user' ' $\mathrm{A}$ ' is clearly far from user ' $C$ ' in the same cell, but it is more close to user ' $B$ ' in the neighbouring cell. Hence, there might be a tendency for user ' $A$ ' to separate from user ' $C$ ' and to join user ' $B$ ', as seen in Fig. 1b of A-Cells. This is also true for the 'boundary user' ' $D$ ' in the northeast cell of Fig. 1a, since it is more close to the users

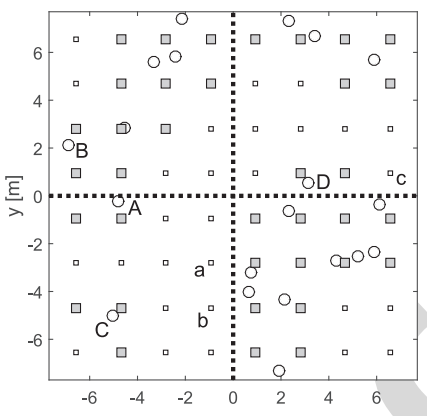

(a) Same number of cells

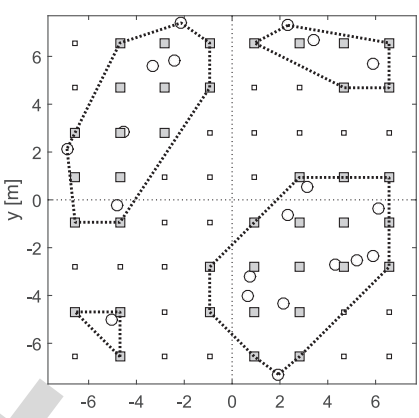

(b) Same number of cells

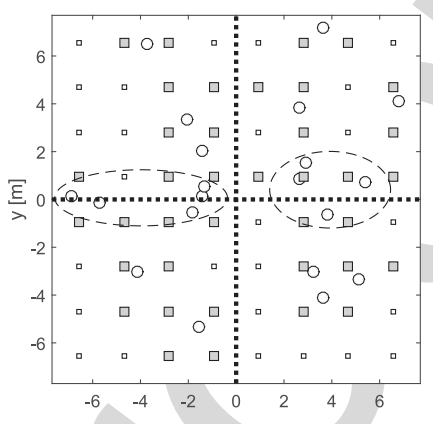

(c) More number of cells

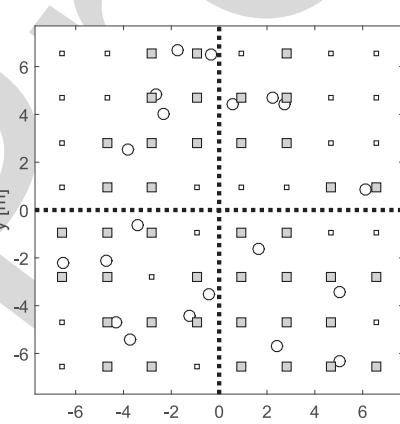

(e) Less number of cells

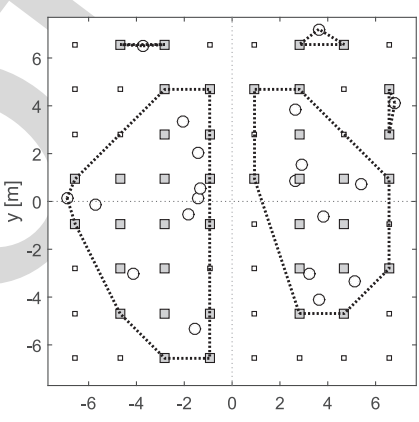

(d) More number of cells

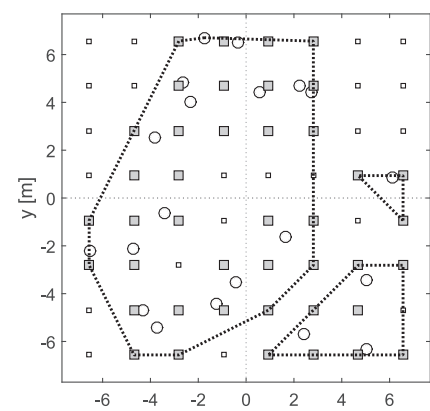

(f) Less number of cells
Fig. 1. Illustration of the conventional (left) and the amorphous structure (right) for VLC indoor systems.

located in the southeast cell, than to the rest of the distant users 237 in the same cell. More examples are shown in Fig. 1c, where 238 two clusters of boundary users highlighted by ellipses join A- 239 Cells of Fig. 1d. In addition to a different user-to-network asso- 240 ciation, the status of APs is also different, where for example, 241 APs ('a', 'b', 'c') were switched from idle mode in conventional 242 cells of Fig. 1a to become active in the A-Cell of Fig. 1b, since 243 they have LoS connections to the associated users. Hence, the 244 proposed A-Cells are capable of breaking boundaries, lead- 245 ing to a higher and a lower number of A-Cells in Fig. 1d 246 and Fig. 1f.

3) Construction of A-Cells: Let us first introduce some 248 common notations. We let $\mathcal{C}$ be the specific set hosting all cells, 249 where for the $c$ th cell $\mathcal{C}_{c}$, we have $\left|\mathcal{N}_{c}\right|$ APs hosted in the set $\mathcal{N}_{c} \quad 250$ serving $\left|\mathcal{K}_{c}\right|$ users of the set $\mathcal{K}_{c}$, with $|\cdot|$ being the cardinality 251 of a set. Note that these notations are in generic sense, includ- 252 ing both conventional cells and A-Cells. We are now ready to 253 discuss the construction of A-Cells.

We firstly construct a full user-to-network association matrix 255 $\boldsymbol{M}^{f}$ having $K$ rows and $N$ columns corresponding to $K$ users 256 
TABLE I

LIST OF PARAMETERS

\begin{tabular}{|l|l|}
\hline \multicolumn{2}{|c|}{ LED-related Parameters } \\
\hline semi-angle at half-illumination $\phi_{1 / 2}$ & $60^{\circ}$ \\
gain of optical filter $f_{\text {of }}(\psi)$ & 1 \\
physical area for a PD receiver $A_{P D}$ & $1 \mathrm{~cm}^{2}$ \\
O/E conversion factor $\gamma$ & $0.53 \mathrm{~A} / \mathrm{W}$ \\
refractive index $n_{r}$ & 1.5 \\
half of the receiver's FoV $\psi_{F}$ & $45^{\circ}$ \\
Vishay TSHG8200 LED min optical power $P_{\text {min }}$ & $5 \mathrm{~mW}$ \\
Vishay TSHG8200 LED max optical power $P_{\text {max }}$ & $50 \mathrm{~mW}$ \\
optical power to luminous flux conversion factor $\delta$ & $2.1 \mathrm{~mW} / \mathrm{lm}$ \\
\hline \hline \multicolumn{2}{|c|}{ Environment-related Parameters } \\
\hline room size & $15 \times 15 \times 3 \mathrm{~m}^{3}$ \\
user height & $0.85 \mathrm{~m}$ \\
number of users (change in Fig 5) & $20(\mathrm{uniform})$ \\
AP height & $2.5 \mathrm{~m}$ \\
number of APs & $8 \times 8(\mathrm{uniform})$ \\
LED array per AP & $15 \times 15$ \\
reflection efficiency $\rho$ & 0.75 \\
illumination requirement [J $\mathcal{J}_{\text {min }}, \mathcal{J}_{\text {max }}, \mathcal{J}_{\text {avg }}$ ] & {$[200,800,600]$} \\
modulation bandwidth $B$ & $20 \mathrm{MHz}$ \\
blocking probability $p$ (change in Fig 4) & 0 \\
\hline \hline \multicolumn{2}{|c}{ Baseline Algorithm-related Parameters } \\
\hline maximum iterations $\varsigma$ (change in Fig 6) & 20 \\
convergence threshold $\zeta$ & 0.01 \\
\hline
\end{tabular}

and $N \mathrm{APs}^{2}$. The $[k, n]$ th entry of $\boldsymbol{M}^{f}$ is set to the LoS channel $h_{k, n}^{0}$. We then carry out $A P$ anchoring as follows:

1) Initialise user-to-network association matrix $\boldsymbol{M}=\boldsymbol{M}^{f}$.

2) Find the best user-AP pair $\left[k^{*}, n^{*}\right]$ having the strongest LoS channel amongst all entries of $\boldsymbol{M}$ and then collect the best user-index $k^{*}$ in $\boldsymbol{k}^{*}$.

3) Set all entries in the $k^{*}$ th row and in the $n^{*}$ th column of $\boldsymbol{M}$ to zero in order to exclude them from further consideration throughout the AP anchoring process.

4) If there are still positive entries in $\boldsymbol{M}$, we repeat this process from Step 2. Otherwise, we output $\boldsymbol{M}$ as the sub-matrix of $\boldsymbol{M}^{f}$ constituted by all rows from $\boldsymbol{k}^{*}$.

The objective of AP anchoring is to have exclusive user-AP pairs ensuring that each of those users in $\boldsymbol{k}^{*}$ will be served at least by its own anchor AP. Those users who have not found their anchor APs will be scheduled during the next anchoring round, however scheduling is beyond our current scope.

Having the user-to-network association matrix $\boldsymbol{M}$, the distance based A-Cells are constructed, where the users are firstly grouped based on a pre-defined distance threshold $d_{0}$ and then we select APs associated with those users ${ }^{3}$ as follows:

1) Introduce the counter $c$, which is initialised as $c=1$.

2) We commence forming cell $\complement_{c}$ by recruiting the first user, who has not been included in any cells. Hence, this user will be the only one in the set $\mathcal{K}_{c}$ and along with the

\footnotetext{
${ }^{2}$ The availability of the full user-to-network association matrix rely on the acquisition of channel knowledge at the AP side, which can be readily estimated at the user side and then fed back to the AP at the cost of a modest overhead. This is because the VLC channels are pre-dominantly static and the channel knowledge can be characterised by a single attenuation factor.

${ }^{3}$ When constructing distance based A-Cells, the mutual distances between users are required. After acquiring the channel knowledge, the distances between users and APs can be inferred from (3). As a result, classical positioning may be used for determining the users' positions. Hence, the mutual distances between users can be readily calculated.
}

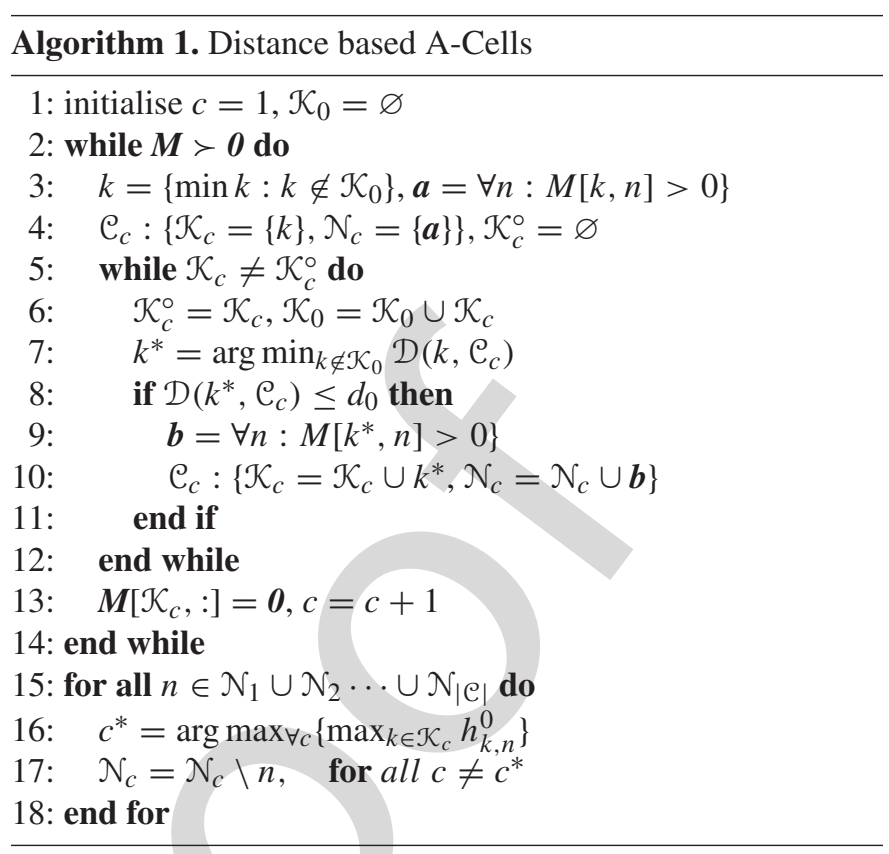

associated AP set $\mathcal{N}_{c}$ containing all the APs providing 282

LoS connections to the first user.

3) Recruit another user from the set of hitherto unas- 284 signed users, who has the smallest distance from the 285 edge/centroid of the cell $\mathfrak{C}_{c}$, provided that their distance is 286 shorter than $d_{0}$. In this step, the edge of the cell is charac- 287 terised by connecting its containing users' positions. We 288 then update cell $\mathrm{C}_{c}$, which results in the expanded set of 289 $\mathcal{K}_{c}$ and $\mathcal{N}_{c}$.

4) We repeat Step 3 until no additional users can be grouped. 291 As a result, we completed the update of cell $\mathcal{C}_{c}$, which 292 resulted in the final set of $\mathcal{K}_{c}$ and $\mathcal{N}_{c}$.

5) Set all entries of the association matrix $\boldsymbol{M}$ associated with 294 the users in $\mathcal{K}_{c}$ to zero. If there are still positive entries in 295 $\boldsymbol{M}$, we increment $c$ and repeat from Step 2. Upon the completion of user grouping, we have to resolve the 297 associated AP ambiguity, since some cells may have conflicting 298 AP assignments. In those cases, any ambiguously assigned AP 299 $n$ is exclusively included in the $c^{*}$ th cell containing those users 300 to whom the ambiguously assigned AP $n$ has the strongest $\operatorname{LoS} 301$ connection. Finally, note that the entire set of APs $\mathcal{N}_{a}$ involved 302 in the ultimately constructed cells, namely $\mathcal{N}_{a}=\mathcal{N}_{1} \cup \mathcal{N}_{2} \cdots \cup 303$ $\mathcal{N}_{|\mathcal{C}|}$, constitutes a subset of whole set of available APs in the 304 room. For those unassigned APs in $\overline{\mathcal{N}}_{a}$, no communications are 305 activated. To ease understanding, we include the pseudo-code in 306 Algorithm 1. Regarding the complexity imposed, the first step 307 of AP anchoring requires only an order of $\mathcal{O}(K)$ operations and 308 an order of $\mathcal{O}(K N \log (K N))$ for finding the best user-AP pair. 309 Furthermore, the complexity of Algorithm 1 is linear within the 310 range of $\left[\mathcal{O}\left(K^{2}\right), \mathcal{O}\left(K^{3}\right)\right]$ owing to its hierarchical algorithmic 311 structure.

\section{B. Link-level Transmission}

Let us now discuss the transmission strategy relying on the 314 amalgamation of ACO-OFDM and MISO transmission. Our 315 
forthcoming elaborations are equally applicable both to the conventional cells and to the proposed A-Cells.

1) ACO-OFDM: We first consider the downlink ACOOFDM transmission from the $n$th $\operatorname{AP} n \in \mathcal{N}_{c}$ to the $k$ th user $k \in \mathcal{K}_{c}$ in the $c$ th cell $\mathcal{C}_{c}$. Let $N_{s}$ represent the number of ACO-OFDM sub-channels and let $\boldsymbol{s}_{n} \in \mathbb{C}^{N_{s} / 4}$ represent the Frequency Domain (FD) information-bearing symbol vector transmitted from the $n$th AP. Then the resultant ACO-OFDM symbol vector $s_{n}^{f} \in \mathbb{C}^{N_{s}}$ is constituted by the odd sub-channel entries of zero and the even sub-channel entries of

$$
s_{n}^{f}[m]= \begin{cases}s_{n}[m / 2] & \text { if } m \leq N_{s} / 2 \text { and is even } \\ s_{n}^{\operatorname{conj}^{[}\left[\frac{N_{s}-m+2}{2}\right]} & \text { if } m>N_{s} / 2 \text { and is even. }\end{cases}
$$

It becomes plausible that the ACO-OFDM mapping of (6) obeys the Hermitian symmetry property, which allows us to create real-valued Time Domain (TD) signal samples $\boldsymbol{s}_{n}^{t} \in \mathbb{R}^{N_{s}}$ after the classic IFFT operation. Since the odd-indexed FD subchannels are set to zero in the specific ACO-OFDM mapping of (6), the first-half of the TD signal samples are copied to the second-half of the TD signal samples, albeit with their signs flipped. As a result, the TD signal samples can be conveyed with all the negative parts clipped at zero. The clipping-distortion imposed by the removal of the negative amplitudes only occurs at the odd-indexed FD sub-channels carrying no data and hence can be ignored, despite the fact that the amplitude of the TD signal samples is halved.

The clipped positive and real-valued TD signal samples $\tilde{\boldsymbol{s}}_{n}^{t} \in$ $\mathbb{R}_{+}^{N_{s}}$ in conjunction with the DC-bias current are then forwarded to the LEDs, where the TD signal envelope is used for modulating the intensity of the LEDs. To elaborate a little further, the clipped TD signal samples $\tilde{\boldsymbol{s}}_{n}^{t}$ obey the clipped Gaussian distribution with a parameter of $\sigma_{s_{n}^{t}}$. Hence, the average optical power of the TD signal samples $\tilde{\boldsymbol{s}}_{n}^{t}$ is $P^{\circ}=\sigma_{s_{n}^{t}} / \sqrt{2 \pi}$ and the total radiated optical power plus DC is $P=P^{d c}+P^{\circ}$. Correspondingly, the average electronic power of the TD signal samples $s_{n}^{t}$ is $P_{t}^{e}=\sigma_{s_{n}^{t}}^{2}$, while the average electronic power of the FD ACO-OFDM symbol vector associated with its even-order half is $P_{f}^{e}=2 P_{t}^{e}$.

The $k$ th user's receiver is comprised of a PD and a transimpedance amplifier. After the removal of DC component and followed by direct detection relying on the Optical to Electrical $(\mathrm{O} / \mathrm{E})$ conversion factor of $\gamma$, the discrete-time model of the received FD symbol vector $\boldsymbol{y}_{k} \in \mathbb{C}^{N_{s}}$ after FFT becomes

$$
\boldsymbol{y}_{k}=(\gamma / 2) \operatorname{diag}[\boldsymbol{h}] \boldsymbol{s}_{n}^{f}+\boldsymbol{I}_{k}+\boldsymbol{w}_{k},
$$

where $\boldsymbol{h} \in \mathbb{R}^{N_{s}}$ hosts the FD channel responses. Since the ACO-OFDM VLC channels can be safely considered as being non-dispersive for a bandwidth $B$ upto $20 \mathrm{MHz}$ [30], each entry of $\boldsymbol{h}$ becomes a single-tap gain factor $h_{k, n}{ }^{4}$. Furthermore, $\boldsymbol{I}_{k} \in$ $\mathbb{C}^{N_{s}}$ denotes the interference imposed on the $k$ th user. Finally, $\boldsymbol{w}_{k} \in \mathbb{C}^{N_{s}}$ represents the noise vector accounting for both the shot noise and the thermal noise at the receiver, which can be

\footnotetext{
${ }^{4}$ We consider white LEDs constructed by using blue LEDs having a phosphor layer, which have a typical bandwidth of $20 \mathrm{MHz}$. This is achievable if a blue filter is used at the receiver side. Our methodology is also eminently applicable, when LEDs with $\geq 100 \mathrm{MHz}$ bandwidth become available.
}

modelled as zero-mean complex-valued AWGN with a vari- 363 ance of $\sigma^{2}=N_{0} B$, where $N_{0} \approx 10^{-22} \mathrm{~A}^{2} / \mathrm{Hz}$ [14]. Extracting 364 the information-bearing sub-channels from $\boldsymbol{y}_{k}$, for each $m \in 365$ $\left\{2,4, \ldots, N_{s} / 2\right\}$ we have,

$$
y_{k}[m]=(\gamma / 2) h_{k, n} s_{n}^{f}[m]+I_{k}[m]+w_{k}[m] .
$$

Remark II-B1: Energy efficiency maximisation of conven- 367 tional RF systems typically relies on Shannon's capacity 368 expression for $f_{t}(\cdot)$ in (2), assuming Gaussian-distributed 369 signalling. However, optical systems relying on Intensity 370 Modulation/Direct Detection (IM/DD) can only have real- 371 valued positive signals. Unfortunately, there is a lack of exact 372 capacity expressions for optical systems, despite the existence 373 of various forms of capacity bounds [31]. Hence, we con- 374 sider ACO-OFDM, since it is a widely used optical modulation 375 scheme exhibiting a higher power efficiency than DCO-OFDM, 376 which allows us to derive an analytically tractable capacity 377 expression [32], [33] for our energy efficiency maximisation 378 problem to be discussed in Section II-C. Suffice to say that, the 379 capacity expression of DCO-OFDM has to take into consider- 380 ation the non-linear clipping distortion effects [33], which are 381 hence set aside for our future research.

2) MISO Transmission: Having discussed the above point- 383 to-point scenario, let us now discuss the multi-user scenario. 384 For the $c$ th cell having $\left|\mathcal{N}_{c}\right|$ APs and $\left|\mathcal{K}_{c}\right|$ users, the equivalent 385 physical layer may be modelled as a multi-user MISO system 386 for $\left|\mathcal{K}_{c}\right|>1$ or as a single-user MISO system for $\left|\mathcal{K}_{c}\right|=1.387$ In the former case, we employ VT based on Zero Forcing 388 (ZF) Transmit Pre-Coding (TPC) for eliminating the inter-user- 389 interference within the $c$ th cell, while for the latter case, we 390 employ CT for maximising the received signal power.

When $\left|\mathcal{K}_{c}\right|=1$, CT is employed, where we transmit the 392 same signal $s_{n}^{f}[m]=x_{k_{0}}[m]$ from all $n \in \mathcal{N}_{c}$ APs to the only 393 user $k_{0} \in \mathcal{K}_{c}$. The signals arriving at the $k_{0}$ th receiver can be 394 constructively combined, since all the channels emerging from 395 all $\mathcal{N}_{c}$ APs to the user $k_{0}$ are positive. Hence, for the $m$ th sub- 396 channel $m \in\left\{2,4, \ldots, N_{S} / 2\right\}$, we have the single-user MISO 397 expression of (8) written as

$$
y_{k_{0}}[m]=(\gamma / 2) \sum_{n \in \mathcal{N}_{c}} h_{k_{0}, n} \varrho_{n}^{c t} x_{k_{0}}[m]+I_{k_{0}}[m]+w_{k_{0}}[m],
$$

where we set normalised electronic power of $x_{k_{0}}[m]$ being unity 399 and let $\varrho_{n}^{c t}=\sqrt{2 P_{c, n}^{c t}}$ with $P_{c, n}^{c t}$ to be optimised. By contrast, 400 for $\left|\mathcal{K}_{c}\right|>1$, VT is employed. For the $m$ th sub-channel $m \in 401$ $\left\{2,4, \ldots, N_{s} / 2\right\}$, we have the multi-user MISO expression of 402 (8) written as

$$
\boldsymbol{y}[m]=(\gamma / 2) \boldsymbol{H s}^{f}[m]+\boldsymbol{I}[m]+\boldsymbol{w}[m],
$$

where $\boldsymbol{y}[m] \in \mathbb{C}^{\left|\mathcal{K}_{c}\right|}$ is the received FD symbol vector of all the 404 $\left|\mathcal{K}_{c}\right|$ users in the $c$ th cell on the $m$ th sub-channel, while $\boldsymbol{s}^{f}[m] \in 405$ $\mathbb{C}^{\left|\mathcal{N}_{c}\right|}$ is the FD symbol vector transmitted from all $\left|\mathcal{N}_{c}\right|$ APs in 406 the $c$ th cell on the $m$ th sub-channel. Finally, $\boldsymbol{H} \in \mathbb{R}^{\left|\mathcal{K}_{c}\right| \times\left|\mathcal{N}_{c}\right|} 407$ is the channel between the $\left|\mathcal{K}_{c}\right|$ users and the $\left|\mathcal{N}_{c}\right|$ APs, while 408 $\boldsymbol{I}[m] \in \mathbb{C}^{\left|\mathcal{K}_{c}\right|}$ is the inter-cell-interference imposed on all $\left|\mathcal{K}_{c}\right| \quad 409$ users in the $c$ th cell on the $m$ th sub-channel. To eliminate the 410 inter-user-interference, we employ TPC, which is formulated 411 
as $\boldsymbol{s}^{f}[m]=\boldsymbol{G} \boldsymbol{x}[m]$, where $\boldsymbol{x}[m] \in \mathbb{C}^{\left|\mathcal{K}_{c}\right|}$ is the multi-user FD symbol vector and the ZF precoding $\boldsymbol{G} \in \mathbb{R}^{\left|\mathcal{N}_{c}\right| \times\left|\mathcal{K}_{c}\right|}$ is explicitly formulated as $\boldsymbol{G}=\boldsymbol{H}^{\dagger}$, which is the pseudo-inverse of $\boldsymbol{H}$. Hence, (10) can be decomposed into $\left|\mathcal{K}_{c}\right|$ parallel streams and for any $k \in \mathcal{K}_{c}$, yielding

$$
y_{k}[m]=(\gamma / 2) \varrho_{k}^{v t} x_{k}[m]+I_{k}[m]+w_{k}[m],
$$

where we set normalised electronic power of $x_{k}[m]$ being unity and let $\varrho_{k}^{v t}=\sqrt{2 P_{c, k}^{v t}}$ with $P_{c, k}^{v t}$ to be optimised.

Remark II-B2: It is natural to consider multi-user MISO transmission in VLC systems using VT to eliminate the interuser-interference. The roots of VT are in the celebrated results of information theory, where Dirty Paper Coding (DPC) is found to be capable of achieving the broadcast channel's capacity [34]. Owing to its non-linear complex nature of implementing DPC, the low-complexity ZF constitutes a popular alternative, which was shown to exhibit a negligible performance loss compared to DPC in the high Signal to Noise Ratio (SNR) regime, when the number of APs is larger than the number of users [35]. Hence, this important theoretical finding benefits directly a range of modern communications systems. For example, the concept of VT is similar to the successful employment of 'vectoring' in the state-of-the-art Digital Subscriber Line (DSL) based G.fast system invoked for coping with the crosstalk between twisted pairs. This operation is also reminiscent of the concept of the Coordinated Multiple Point (CoMP) transmission regime of classic RF cellular communications conceived for mitigating the inter-cell-interference at the cell edge [36]. In practice, to facilitate VT from $\left|\mathcal{N}_{c}\right|$ APs to $\left|\mathcal{K}_{c}\right|$ users, both the channel matrix $\boldsymbol{H}$ and the users' data $\boldsymbol{x}[\mathrm{m}]$ have to be shared amongst the $\left|\mathcal{N}_{c}\right|$ APs. Fortunately, this requirement can be satisfied, since the VLC channels are prepredominately static, while the sharing of all users' data $\boldsymbol{x}[\mathrm{m}]$ requires a more capable back-haul.

3) Optical Constraints: Typically, the forward current of the DC-biased and clipped TD signal samples should be within the LED's dynamic range [30]. Since the total radiated optical power is directly proportional to the forward current, we describe the optical constraints in terms of their optical power.

The total optical power radiated from an AP should satisfy the per-LED dynamic range of $P_{\min } \leq P / L \leq P_{\max }$, where we assume that each of the $L$ LEDs constituting an AP emits the same optical power. For example, a practical dynamic range of a Vishay TSHG8200 LED is between $P_{\min }=5 \mathrm{~mW}$ and $P_{\max }=50 \mathrm{~mW}$ at room temperature. For satisfying a predefined illumination requirement constituted by the minimum illumination $\mathcal{J}_{\min }$, the maximum illumination $\mathcal{J}_{\text {max }}$ and the average illumination $\mathcal{J}_{\text {avg }}$, we find the minimum required optical power $P_{\min }^{\text {illu }}$ by solving the problem of

$$
\begin{gathered}
P_{\text {min }}^{\text {illu }}=\min P \quad \text { s.t. } \\
\min _{\mu \in\left[1, K_{p}\right]} \sum_{n=1}^{N} h_{\mu, n}^{\text {illu }} P \geq \mathcal{J}_{\text {min }}, \quad \max _{\mu \in\left[1, K_{p}\right]} \sum_{n=1}^{N} h_{\mu, n}^{\text {illu }} P \leq \mathcal{J}_{\text {max }}, \\
\mathcal{J}_{\text {avg }}^{-} \leq \frac{1}{K_{p}} \sum_{\mu=1}^{K_{p}} \sum_{n=1}^{N} h_{\mu, n}^{\text {illu }} P \leq \mathcal{J}_{\text {avg }}^{+}, \quad P_{\text {min }} \leq P / L \leq P_{\text {max }},
\end{gathered}
$$

We may also define a global energy efficiency as the sum 494 throughput of all cells divided by the total power consump- 495 tion of all cells, where a centralised approach has to be used. 496 By contrast, the per-cell basis definition of (16) supports a 497

\footnotetext{
${ }^{5}$ We fix the DC-biasing by focusing our attention on the communicationsrelated energy efficiency maximisation, despite the possibility of adaptive signal scaling and DC biasing [37]. Note that, when DCO-OFDM is considered, optimising the DC-bias component becomes critical [30].
} denotes the luminous flux of the unit optical power provided 460 by the $n$th $\mathrm{AP}$ at the $\mu$ th point of the $K_{p}$ equally partitioned 461 receiver plane, which is given as

where $\delta$ denotes the optical power to luminous flux conversion 463 factor [14]. Similarly, we also find the maximum optical power 464 $P_{\max }^{i l l u}=\max P$ capable of satisfying the constraints of (12). 465 Note that having predefined illumination requirements also pre- 466 absence of any further clipping at the receiver. As a result, 468 LED's physical limits, we have the optical constraint of

In this paper, we fix the DC-bias component ${ }^{5}$ and assume 471 only negative clipping is incurred by our ACO-OFDM scheme, 472 because we can always set an appropriate margin for prevent- 473 ing upper clipping imposed by the high Peak to Average Power 474 Ratio (PAPR) of ACO-OFDM TD signal samples by control- 475 ling the maximum optical output power. More explicitly, to 476 avoid insufficient forward biasing, we set the DC-bias com- 477 ponent to be at least as high as the minimum optical power 478 required for satisfying the LED's dynamic range. On the other 479 hand, we also adjust the DC-bias component to 'just' sat- 480 isfy the predefined illumination requirement. Hence, we have 481 $P^{d c} / L=\max \left\{P_{\min }^{i l l u}, P_{\min }\right\}$ and the optical requirement of (14) 482 becomes $0 \leq P^{\circ} / L \leq P_{\max }^{\circ}$, where

represents the maximum tolerable additional optical power 484 of each LED, so that the communication function would not 485 violate the illumination and LED instrument requirements.

Let us now discuss the system-level energy efficiency max- 488 imisation. Our forthcoming elaborations are equally applicable 489 both to the conventional cells and to the proposed A-Cells. $\quad 490$

1) Formulation: Let us formulate our energy efficiency 491 maximisation problem by defining system's mean energy effi- 492 ciency on a per-cell basis as

$$
\max _{\mathcal{P}} \mathrm{EE}=\frac{1}{|\mathfrak{C}|} \sum_{\mathrm{c}} \mathrm{EE}_{\mathrm{c}}(\mathcal{P}),
$$
vents the saturation of the PD receiver, hence we assume the 467 by taking into account both the illumination requirements and 469 
more scalable and efficient distributed approach. Since $|\mathfrak{C}|$ is a constant, (16) can be solved equivalently by optimising $\max _{\mathcal{P}} \sum_{c} \mathrm{EE}_{\mathrm{c}}(\mathcal{P})$, explicitly

$$
\max _{\boldsymbol{P}} \sum_{c \in \mathcal{C}_{v t}} \frac{f_{t, c}^{v t}(\boldsymbol{P})}{f_{p, c}^{v t}\left(\boldsymbol{P}_{c}^{v t}\right)}+\sum_{c \in \mathfrak{C}_{c t}} \frac{f_{t, c}^{c t}(\boldsymbol{P})}{f_{p, c}^{c t}\left(\boldsymbol{P}_{c}^{c t}\right)} \quad \text { s.t. } \quad \mathrm{C} 1, \mathrm{C} 2
$$

where $\mathcal{C}_{v t}$ and $\mathcal{C}_{c t}$ are the sets hosting the specific cells that employ VT and CT, respectively. Furthermore, we have $\boldsymbol{P}=$ $\left\{\boldsymbol{P}^{v t} ; \boldsymbol{P}^{c t}\right\}$ acting as the power allocation strategy, which is constituted by that of all VT-aided cells $\boldsymbol{P}^{v t}=\left\{\boldsymbol{P}_{c}^{v t}, \forall c \in \mathcal{C}_{v t}\right\}$ and all CT-aided cells $\boldsymbol{P}^{c t}=\left\{\boldsymbol{P}_{c}^{c t}, \forall c \in \mathcal{C}_{v t}\right\}$, where $\boldsymbol{P}_{c}^{v t}$ and $\boldsymbol{P}_{c}^{c t}$ are the power allocation strategy for the $c$ th cell using VT and CT, respectively. By scrutinising (17), we find that only those cells in $\mathrm{C}_{v t}$ and $\mathrm{C}_{c t}$ are included in our optimisation. This implies that those APs, which are not included in $\mathcal{C}_{v t}$ and $\mathcal{C}_{c t}$, are switched into their idle mode.

Still referring to (17), since ACO-OFDM is employed, we can assume Gaussian signalling for $x_{k}[\mathrm{~m}]$ in (11) and for $x_{k_{0}}[\mathrm{~m}]$ in (9). Hence, we arrive at

$$
\begin{aligned}
& f_{t, c}^{v t}(\boldsymbol{P})=\sum_{k \in \mathcal{K}_{c}} \underbrace{\kappa \log _{2}\left[1+\frac{\left(\gamma^{2} / 2\right) P_{c, k}^{v t}}{\sigma^{2}+I_{c, k}\left(\boldsymbol{P}_{\bar{c}}^{v t}, \boldsymbol{P}^{c t}\right)}\right]}_{f_{t, c, k}^{v t}\left(P_{c, k}^{v t}, \boldsymbol{P}_{\bar{c}}^{v t}, \boldsymbol{P}^{c t}\right)}, \\
& f_{t, c}^{c t}(\boldsymbol{P})=\kappa \log _{2}\left[1+\frac{\left(\gamma^{2} / 2\right)\left(\sum_{n \in \mathcal{N}_{c}} h_{k_{0}, n} \sqrt{P_{c, n}^{c t}}\right)^{2}}{\sigma^{2}+I_{c, k_{0}}\left(\boldsymbol{P}_{\bar{c}}^{c t}, \boldsymbol{P}^{v t}\right)}\right],
\end{aligned}
$$

where $\kappa=(1-p) B / 4$ is a constant, with the factor of 4 representing the bandwidth efficiency loss owing to the employment of ACO-OFDM having a bandwidth of $B$ and $p$ denoting the blocking probability. Furthermore, $I_{c, k}(\cdot)$ denotes the interference imposed on the $k$ th user in the $c$ th VT-aided cell, while $I_{c, k_{0}}(\cdot)$ denotes the interference imposed on the only user $k_{0}$ in the $c$ th CT-aided cell. In (18) and (19), $\boldsymbol{P}_{\bar{c}}^{v t}$ and $\boldsymbol{P}_{\bar{c}}^{c t}$ represent the power allocation excluding the $c$ th cell from all VT-aided and CT-aided cells, respectively, which are used for evaluating the interference terms $I_{c, k}(\cdot)$ and $I_{c, k_{0}}(\cdot)$. Finally, since the DC-bias component is fixed for illumination, we consider the communications-related power consumption in the electronic domain, which may be written as

$$
\begin{aligned}
& f_{p, c}^{v t}\left(\boldsymbol{P}_{c}\right)=\sum_{n \in \mathcal{N}_{c}} \sum_{k \in \mathcal{K}_{c}} g_{n, k}^{2} P_{c, k}^{v t}, \\
& f_{p, c}^{c t}\left(\boldsymbol{P}_{c}\right)=\sum_{n \in \mathcal{N}_{c}} P_{c, n}^{c t},
\end{aligned}
$$

where $g_{n, k}$ is the $[n, k]$ th entry of the TPC matrix $\boldsymbol{G}^{6}$. Furthermore, as far as the optimisation constraints are

\footnotetext{
${ }^{6} \mathrm{We}$ only consider transmission power as our source of power consumption in (20) and (21). In our future work, other sources of power consumption, such as the signal processing costs, back-haul power consumption etc would be desired to paint the whole picture in terms of the entire network power consumption [29]. However, at the time of writing, quantifying the network power consumption for VLC systems remains an open issue, since integrating it with a certain VLC back-haul requires dedicated treatment.
}

concerned, we impose the per-LED optical power constraint 529 pair $\mathrm{C} 1$ of

$$
\begin{aligned}
\frac{1}{L} \sqrt{\sum_{k \in \mathcal{K}_{c}} g_{n, k}^{2} P_{c, k}^{v t} / 2 \pi} & \leq P_{\text {max }}^{\circ} \quad \forall n \in \mathcal{N}_{c}, \forall c \in \mathcal{C}_{v t}, \\
\frac{1}{L} \sqrt{P_{c, n}^{c t} / 2 \pi} & \leq P_{\text {max }}^{\circ} \quad \forall n \in \mathcal{N}_{c}, \forall c \in \mathcal{C}_{c t} .
\end{aligned}
$$

We also impose the per-user throughput constraint pair C2 for 531 guaranteeing a minimum required QoS as

$$
\begin{aligned}
f_{t, c, k}^{v t}\left(P_{c, k}^{v t}, \boldsymbol{P}_{\bar{c}}^{v t}, \boldsymbol{P}^{c t}\right) & \geq R \quad \forall k \in \mathcal{K}_{c}, \forall c \in \mathcal{C}_{v t}, \\
f_{t, c}^{c t}(\boldsymbol{P}) & \geq R \quad \forall c \in \mathcal{C}_{c t} .
\end{aligned}
$$

Note that neglecting the per-user throughput constraint pair C2 533 by unilaterally maximising the energy efficiency results in an 534 ill-defined problem, since the user QoS target is ignored.

2) Transformation: The objective function defined in (17) 536 is complex due to the coupled nature of the power allocation 537 strategy. Hence, we opt for decoupling the original problem 538 formulated in (17) so as to allow efficient distributed process- 539 ing. More explicitly, instead of evaluating the true interference 540 term of (18) and (19), we consider the interference upper bound 541 by assuming that all other cells transmit at their maximum 542 permissible optical power. This implies that we carry out guar- 543 anteed energy efficiency maximisation. More specifically, we 544 use constant $I_{c, k}\left(P_{\max }^{\circ}\right)$ to represent the maximum possible 545 interference imposed on the $k$ th user in the $c$ th VT-aided cell, 546 which is written as

$$
I_{c, k}\left(P_{\text {max }}^{\circ}\right)=\gamma^{2} \pi \sum_{\tilde{c} \in \mathcal{C}, \tilde{c} \neq c} \sum_{n \in \mathcal{N}_{\tilde{c}}} h_{k, n}^{2}\left(L P_{\text {max }}^{\circ}\right)^{2} .
$$

Similarly, by replacing $k=k_{0}$ in (26), we have $I_{c, k_{0}}\left(P_{\text {max }}^{\circ}\right)$ rep- 548 resenting the maximum possible interference imposed on the 549 only user $k_{0}$ in the $c$ th CT-aided cell. Correspondingly, we can 550 reformulate the lower bound of (18) and (19) as

$$
\begin{aligned}
& f_{t, c}^{v t, l}\left(\boldsymbol{P}_{c}^{v t}\right)=f_{t, c}^{v t}(\boldsymbol{P})_{\left\{I_{c, k}\left(\boldsymbol{P}_{\bar{c}}^{v t}, \boldsymbol{P}^{c t}\right) \mapsto I_{c, k}\left(P_{\max }^{\circ}\right), \forall k \in \mathcal{K}_{c}\right\}}, \\
& f_{t, c}^{c t, l}\left(\boldsymbol{P}_{c}^{c t}\right)=f_{t, c}^{c t}(\boldsymbol{P})_{\left\{I_{c, k_{0}}\left(\boldsymbol{P}_{\bar{c}}^{c t}, \boldsymbol{P}^{v t}\right) \mapsto I_{c, k_{0}}\left(P_{\text {max }}^{\circ}\right)\right\}} \cdot
\end{aligned}
$$

It is plausible that (27) and (28) become only the function of 552 the power allocation strategy of the $c$ th cell, which is decoupled 553 from other cells. Furthermore, since the composition in (19) or 554 in its lower bound expression of (28) results in a non-concave 555 function, we rely on the lower bounding of (28) in order to 556 arrive at the concave formulation of

$$
f_{t, c}^{c t, l l}\left(\boldsymbol{P}_{c}^{c t}\right)=\kappa \log _{2}\left[1+\frac{\left(\gamma^{2} / 2\right) \sum_{n \in \mathcal{N}_{c}} h_{k_{0}, n}^{2} P_{c, n}^{c t}}{\sigma^{2}+I_{c, k_{0}}\left(P_{\text {max }}^{\circ}\right)}\right] .
$$

Upon taking into account (27) and (29), we arrive at the new 558 constraint pair $\mathrm{C} 2$ formulated as

$$
\begin{aligned}
f_{t, c, k}^{v t, l}\left(P_{c, k}^{v t}\right) & \geq R \quad \forall k \in \mathcal{K}_{c}, \forall c \in \mathcal{C}_{v t}, \\
f_{t, c}^{c t, l l}\left(\boldsymbol{P}_{c}^{c t}\right) & \geq R \quad \forall c \in \mathcal{C}_{c t} .
\end{aligned}
$$

Clearly, this new constraint pair is more strict than the origi- 560 nal constraint pair of (24) and (25). Lastly, the constraint pair 561 
C1 remains the same, as in (22) and (23). Following the above transformation, the decoupled energy efficiency maximisation problem becomes

$$
\max _{\boldsymbol{P}} \sum_{c \in \mathcal{C}_{v t}} \frac{f_{t, c}^{v t, l}\left(\boldsymbol{P}_{c}^{v t}\right)}{f_{p, c}^{v t}\left(\boldsymbol{P}_{c}^{c t}\right)}+\sum_{c \in \mathfrak{C}_{c t}} \frac{f_{t, c}^{c t, l l}\left(\boldsymbol{P}_{c}^{c t}\right)}{f_{p, c}^{c t}\left(\boldsymbol{P}_{c}^{c t}\right)}
$$

$$
\text { s.t. (22), (23), (30), (31). }
$$

Hence, the problem formulated in (32) can be solved in form of $|\mathrm{C}|$ parallel sub-problems, where we have

$$
\begin{aligned}
& \max _{\boldsymbol{P}_{c}^{v t}} \frac{f_{t, c}^{v t, l}\left(\boldsymbol{P}_{c}^{v t}\right)}{f_{p, c}^{v t}\left(\boldsymbol{P}_{c}^{v t}\right)} \quad \text { s.t. } \quad(22),(30) \quad \forall c \in \mathcal{C}_{v t}, \\
& \max _{\boldsymbol{P}_{c}^{c t}} \frac{f_{t, c}^{c t, l l}\left(\boldsymbol{P}_{c}^{c t}\right)}{f_{p, c}^{c t}\left(\boldsymbol{P}_{c}^{c t}\right)} \quad \text { s.t. } \quad(23),(31) \quad \forall c \in \mathcal{C}_{c t} .
\end{aligned}
$$

Both (33) and (34) constitute fractional programming problems, which may be solved using Dinkelbach's method [38] by iteratively optimise the subtractive form of (33) and (34). More explicitly, for a particular $c \in \mathcal{C}_{v t}$, the procedures are

1) Introduce the parameter $t_{c, i}^{v t}$ with $i$ being the iteration index and initialise the parameter as $t_{c, 1}^{v t}=0$.

2) At each iteration $i$, solve the inner optimisation problem of the subtractive form of (33), namely

$$
\max _{\boldsymbol{P}_{c}^{v t}} f_{t, c}^{v t, l}\left(\boldsymbol{P}_{c}^{v t}\right)-t_{c, i}^{v t} f_{p, c}^{v t}\left(\boldsymbol{P}_{c}^{v t}\right) \quad \text { s.t. } \quad(22),(30) .
$$

Since the above inner optimisation problem is a concave maximisation problem, the classic dual-decomposition method yields a zero duality gap and hence achieves optimality. Owing to its popularity in solving convex problems, we refer readers for further details to [39].

3) Let $\boldsymbol{P}_{c}^{v t}$,* denote the optimal solutions found for the inner optimisation problem, if we have

$$
f_{t, c}^{v t, l}\left(\boldsymbol{P}_{c}^{v t, *}\right)-t_{c, i}^{v t} f_{p, c}^{v t}\left(\boldsymbol{P}_{c}^{v t, *}\right) \leq \zeta
$$

then the pre-defined convergence threshold $\zeta$ is satisfied, where $\boldsymbol{P}_{c}^{v t, *}$ constitutes the ultimate solution. Alternatively, if the maximum number of iterations $\zeta$ is reached, we output $\boldsymbol{P}_{c}^{v t, *}$ as the ultimate solution. Otherwise, we update the parameters according to

$$
t_{c, i+1}^{v t}=f_{t, c}^{v t, l}\left(\boldsymbol{P}_{c}^{v t, *}\right) / f_{p, c}^{v t}\left(\boldsymbol{P}_{c}^{v t, *}\right),
$$

and repeat Steps 2 and 3 .

The above procedures, referred to as the baseline algorithm ${ }^{7}$, can be also used for the energy efficiency maximisation of (34) for CT-aided cells and we do not duplicate it here.

3) Simplification: The baseline algorithm requires a number of iterations to converge, where within each iteration, the classic dual-decomposition method invoked, again, requires encapsulated iterations to converge. Hence, we propose an algorithm, which dispenses with the above sophistication. For any

\footnotetext{
${ }^{7}$ Other methods solving fractional programming, such as bisection and Charnes-Cooper method, are also interesting but they are beyond our scope.
}

particular VT-aided cell $c \in \mathcal{C}_{v t}$, we use the straightforward 596 equal power allocation strategy of

$$
P_{c, k}^{v t}=P_{c}^{v t} \quad \forall k \in \mathcal{K}_{c}
$$

To satisfy the constraint (30), we have

$$
\begin{aligned}
P_{c, k}^{v t} & \geq\left(2^{R / \kappa}-1\right)\left[\sigma^{2}+I_{c, k}\left(P_{\text {max }}^{\circ}\right)\right] 2 / \gamma^{2}=P_{c, k}^{v t, l}, \\
P_{c}^{v t} & \geq \max _{k \in \mathcal{K}_{c}} P_{c, k}^{v t, l}=P_{c}^{v t, l},
\end{aligned}
$$

while to satisfy the constraint (22), we have

$$
P_{c}^{v t} \leq 2 \pi\left(L P_{\text {max }}^{\circ}\right)^{2} / \sum_{k \in \mathcal{K}_{c}} g_{n, k}^{2}=P_{c}^{v t, u} .
$$

As a result, provided that $P_{c}^{v t, l} \leq P_{c}^{v t, u}$, we have

$$
P_{c}^{v t}=P_{c}^{v t, l}
$$

The solution $P_{c}^{v t}$ in (41) ensures that even the least privi- 601 leged user $k_{w}=\arg \max _{k \in \mathcal{K}_{c}}\left\{I_{c, k}\left(P_{\text {max }}^{\circ}\right)\right\}$ satisfies the per-user 602 throughput constraint of (30). However, setting $P_{c}^{v t}$ for other 603 users may 'over-satisfy' their per-user throughput constraint 604 of (30), which leads to an energy efficiency loss. Hence, we 605 improve the above equal power allocation strategy as $\quad 606$

1) Let $\mathcal{K}_{c}^{f}$ be the set hosting those users with fixed power 607 allocation strategies, which is initialised as $\mathcal{K}_{c}^{f}=\left\{k_{w}\right\} .608$ Let $\mathcal{K}_{c}^{p}$ be the complement set of $\mathcal{K}_{c}^{f}$ hosting those users 609 with adjustable power allocation strategies. Finally, we 610 initialise $\boldsymbol{A}=\left\{P_{c, k \in \mathcal{K}_{c}}^{v t}=P_{c}^{v t}\right\}$.

2) For $k \in \mathcal{K}_{c}^{p}$, we reduce the power allocated from $P_{c, k}^{v t}=612$ $P_{c}^{v t}$ given in (41) to $P_{c, k}^{v t}=P_{c, k}^{v t, l}$ given in (38). This will 613 result in the adjusted power allocation strategy of

$$
\begin{aligned}
& \boldsymbol{A}_{k}=\left\{P_{c, k}^{v t}=P_{c, k}^{v t, l}, \boldsymbol{\Omega}_{k}\right\}, \\
& \boldsymbol{\Omega}_{k}=\left\{P_{c, j \in \mathcal{K}_{c}^{f}}^{v t}=P_{c, j}^{v t, l}, P_{c, \tilde{k} \in \mathcal{K}_{c}^{p}, \tilde{k} \neq k}^{v t}=P_{c}^{v t}\right\} .
\end{aligned}
$$

3) Repeating Step 2 for all users in $\mathcal{K}_{c}^{p}$ leads to $\left|\mathcal{K}_{c}^{p}\right|$ adjusted 615 power allocation strategies $\left\{\boldsymbol{A}_{k}, k \in \mathcal{K}_{c}^{p}\right\}$, where we find 616 the one gives the highest energy efficiency

$$
k_{p}=\arg \max _{k \in \mathcal{K}_{c}^{p}}\left\{f_{t, c}^{v t, l}\left(\boldsymbol{A}_{k}\right) / f_{p, c}^{v t}\left(\boldsymbol{A}_{k}\right)\right\} .
$$

4) If we have

$$
f_{t, c}^{v t, l}\left(\boldsymbol{A}_{k_{p}}\right) / f_{p, c}^{v t}\left(\boldsymbol{A}_{k_{p}}\right) \geq f_{t, c}^{v t, l}(\boldsymbol{A}) / f_{p, c}^{v t}(\boldsymbol{A}),
$$

then we include $k_{p}$ in $\mathcal{K}_{c}^{f}$ by excluding it from $\mathcal{K}_{c}^{p}$ and 619 set $\boldsymbol{A}=\boldsymbol{A}_{k_{p}}$. Then we repeat the procedure commencing 620 from Step 2. The adjustment stops, when (45) is not met 621 and we output $\boldsymbol{P}_{c}^{v t, *}=\boldsymbol{A}$.

For any particular CT-aided cell $c \in \mathcal{C}_{c t}$, we use the equal 623 power allocation strategy, namely

$$
P_{c, n}^{c t}=P_{c}^{c t} \quad \forall n \in \mathcal{N}_{c} .
$$

To satisfy the constraint (31), we have

$$
P_{c}^{c t} \geq \frac{\left(2^{R / \kappa}-1\right)\left[\sigma^{2}+I_{c, k_{0}}\left(P_{\max }\right)\right]}{\left(\gamma^{2} / 2\right) \sum_{n \in \mathcal{N}_{c}} h_{k_{0}, n}^{2}}=P_{c}^{c t, l},
$$


while to satisfy the constraint (23), we have

$$
P_{c}^{c t} \leq 2 \pi\left(L P_{\max }^{\circ}\right)^{2}=P_{c}^{c t, u} .
$$

As a result, provided that $P_{c}^{c t, l} \leq P_{c}^{c t, u}$, we have

$$
P_{c}^{c t}=P_{c}^{c t, l} \text {. }
$$

Let us now adjust the equal power allocation strategy by turning off the communications function for those APs exhibiting highattenuation channels, since blindly allocating power to it may lead to energy efficiency loss. More explicitly

1) Let $\mathcal{N}_{c}^{0}$ and $\mathcal{N}_{c}^{1}$ be the set hosting the specific APs whose communication functions are turned off and turned on, respectively, where we have $\mathcal{N}_{c}^{0}=\varnothing$ and $\mathcal{N}_{c}^{1}=\mathcal{N}_{c}$. Initialise $\boldsymbol{A}=\left\{P_{c, n \in \mathcal{N}_{c}}^{c t}=P_{c}^{c t}\right\}$.

2) Locate the weakest AP

$$
n_{w}=\arg \min _{n \in \mathcal{N}_{c}^{1}}\left\{h_{k_{0}, n}\right\}
$$

and include it in $\mathcal{N}_{c}^{0}$ by excluding it from $\mathcal{N}_{c}^{1}$.

3) Re-evaluate (47) and (48) using $\mathcal{N}_{c}^{1}$ instead of $\mathcal{N}_{c}$ in order to get $P_{c}^{c t, 1}$. Hence, we arrive at the new power allocation strategy of

$$
\boldsymbol{A}_{n_{w}}=\left\{P_{c, n \in \mathcal{N}_{c}^{0}}^{c t}=0, P_{c, n \in \mathcal{N}_{c}^{1}}^{c t}=P_{c}^{c t, 1}\right\} .
$$

4) If we have

$$
f_{t, c}^{c t, l l}\left(\boldsymbol{A}_{n_{w}}\right) / f_{p, c}^{c t}\left(\boldsymbol{A}_{n_{w}}\right) \geq f_{t, c}^{c t, l l}(\boldsymbol{A}) / f_{p, c}^{c t}(\boldsymbol{A}),
$$

we set $\boldsymbol{A}=\boldsymbol{A}_{n_{w}}$ and repeat the above procedure, commencing from Step 2. The adjustments stop when (52) is not met and we output $\boldsymbol{P}_{c}^{c t, *}=\boldsymbol{A}$.

Remark II-C3: If no power adjustments are carried out, the algorithm proposed for the VT-aided or CT-aided cells only requires a one-off evaluation of (41) or (49), respectively. When adjustments are indeed required, the algorithm proposed requires at most $\left|\mathcal{K}_{c}\right|-1$ or $\left|\mathcal{N}_{c}\right|-1$ additional iterations for the VT-aided or CT-aided cells, respectively. Within each iteration, the dominant complexity component is only linearly increasing according to the order of $\mathcal{O}\left(\mathcal{K}_{c}^{p}\right)$ or $\mathcal{O}\left(\mathcal{N}_{c}^{1}\right)$ associated with evaluating (44) or (50) for the VT-aided or CT-aided cells, respectively. To further aid the reader's understanding, a flow chart is included in Fig. 2.

\section{NUMERICAL RESULTS}

Let us now provide simulation results for characterising the energy efficiency of the indoor VLC system relying on the amorphous structure. All our simulations are carried out for 100 random independent snapshots of the user distributions using the parameters included in Table I.

\section{A. Comparisons}

For fair comparisons, in Fig. 3, the same transmission strategy is employed and the same baseline optimisation algorithm is applied for all the cell formation strategies involved, where we have set the minimum per-user throughput constraint to $R=15 \mathrm{MBits} / \mathrm{s}$ for our optimisation algorithm.

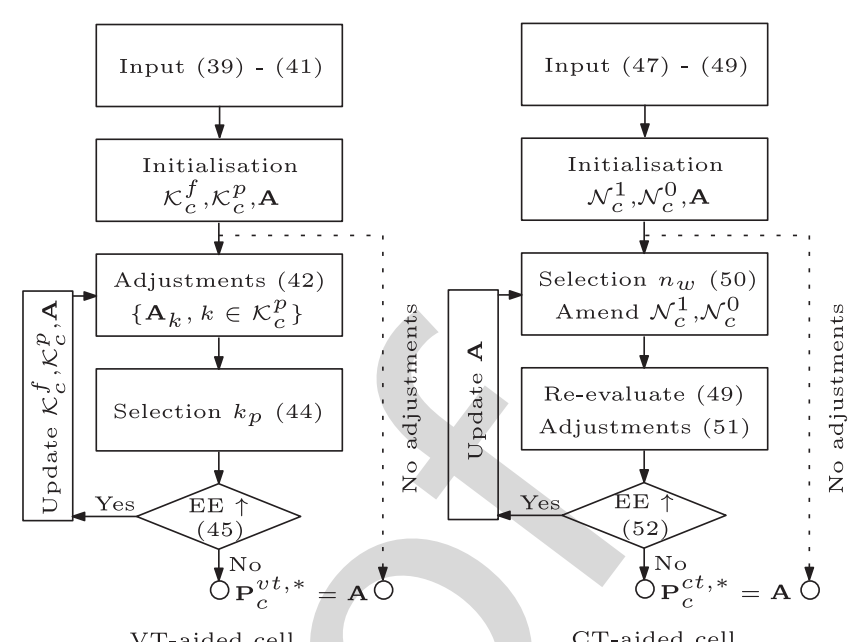

Fig. 2. Flowcharts of the proposed algorithm.

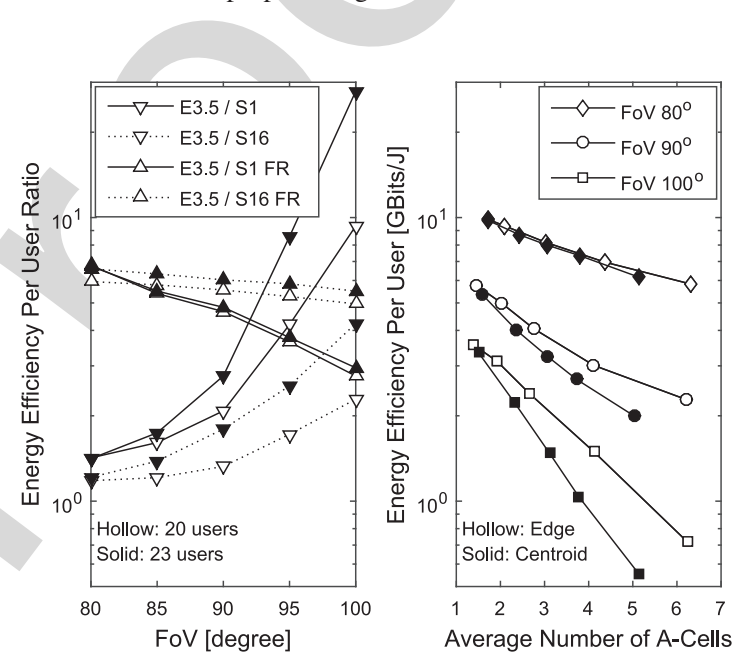

Fig. 3. Energy efficiency per user comparisons of various cell formation strategies (left) as well as between the edge and centroid-distance based A-Cells (right) using the parameters of Table I.

1) Edge-Distance-Based A-Cells vs. Conventional Cells: 668 The left subplot of Fig. 3 compares the energy efficiency 669 per user between the conventional cells and the edge-distance 670 based A-Cells having 20 users (hollow) and 25 users (solid). 671 Explicitly, 'E3.5' stands for the edge-distance based A-Cells 672 having $d_{0}=3.5 \mathrm{~m}$. This value of $d_{0}$ was specifically selected to 673 result in an average number of cells, which is similar to that of 674 the bench-marker scenario 'S16'. We also included the bench- 675 marker scenario ' $\mathrm{S} 1$ ' as introduced in Section II-A2. Since 676 similar trends may also be found for the centroid-distance based 677 A-Cells, we omit them for space economy. 678

It can be seen from the left subplot of Fig. 3 that, for both 679 20 users and 25 users, the edge-distance based A-Cells exhibit 680 a consistently higher energy efficiency per user than both the 681 conventional cell formations ' $\mathrm{S} 16$ ' and 'S1', where the relative 682 energy efficiency becomes significantly greater upon increasing 683 the FoVs when experiencing more interference. Quantitatively, 684 when having 20 users, the edge-distance based A-Cells are 685 capable of achieving over 4 times (nearly 10 times) energy effi- 686 ciency per user than that of the bench-marker scenario 'S1' 687 
at $\mathrm{FoV}$ of $95^{\circ}$ ( of $100^{\circ}$ ). Similarly, when compared to the bench-marker scenario 'S16', the edge-distance based A-Cells are capable of roughly doubling the energy efficiency per user at both FoVs of $95^{\circ}$ and of $100^{\circ}$. These observations imply that the advocated edge-distance based A-Cells are capable of handling scenarios having more interference. This is true upon slightly increasing the number of users from 20 to 25 , where the edge-distance based A-Cells achieve overwhelmingly higher energy efficiency per user than both conventional cell formations 'S16' and 'S1'.

Furthermore, we included the classic scheme of frequency reuse having a factor of two in conjunction with both the conventional cell formations ' $\mathrm{S} 16$ ' and ' $\mathrm{S} 1$ '. It can be seen from the left subplot of Fig. 3 that the edge-distance based A-Cells exhibit a consistently higher energy efficiency per user than both the conventional cell formations relying on frequency reuse for all FoV settings and for both user settings. For all FoVs, as expected, the higher the number of users, the higher the energy efficiency per user of the edge-distance based A-Cells becomes in comparison to both conventional cell formations. Upon increasing the FoVs, the energy efficiency per user achieved by the edge-distance based A-Cells is reduced for both conventional cell formations as well as for both user settings, exhibiting a steeper reduction for the conventional cell formation ' $\mathrm{S} 1$ '. This is because, the higher the FoV, the more interference is encountered, hence frequency reuse becomes more beneficial. Despite this reduction, the energy efficiency per user achieved by the edge-distance based A-Cells remains at least three times higher when compared to that of the conventional cell formation ' $\mathrm{S} 1$ ' employing frequency reuse and supporting 20 users. To sum up, the edge-distance based A-Cells are significantly more energy efficient than both the conventional cell formations ' $\mathrm{S} 16$ ' and ' $\mathrm{S} 1$ ' operating with or without frequency reuse.

2) Edge-Distance vs. Centroid-Distance-Based A-Cells: Owing to the flexibility of the distance based A-Cells, we can appropriately configure them to provide a fair comparison. The right subplot of Fig. 3 compares the energy efficiency per user between the edge-distance (hollow) and the centroid-distance (solid) based A-Cells with the average number of A-Cells spanning from 1 to 6 . For the edge-distance based A-Cells, we evaluate $d_{0}=[3,3.5,4,4.5,5]$, while for the centroid-distance based A-Cells, we evaluate $d_{0}=[5,6,7,8,9]$. Note that the higher the value of $d_{0}$, the smaller the average number of resultant A-Cells. It can be seen from the right subplot of Fig. 3 that the edge-distance based A-Cells exhibit a consistently higher energy efficiency per user than that of the centroid-distance based A-Cells for all resultant average number of A-Cells and for all FoVs, which shows the superiority of the edge-distance based A-Cells. Indeed, we observe that the superiority of the edge-distance based A-Cells is more prominent at higher FoVs for all resultant average number of A-Cells, while they become very similar at FoV of $80^{\circ}$. Finally, at FoVs of $90^{\circ}$ and of $100^{\circ}$, we observe that the superiority of edge-distance based A-Cells is greater when having a larger number of resultant ACells, whilst there are marginal differences when the number of A-Cells is small.
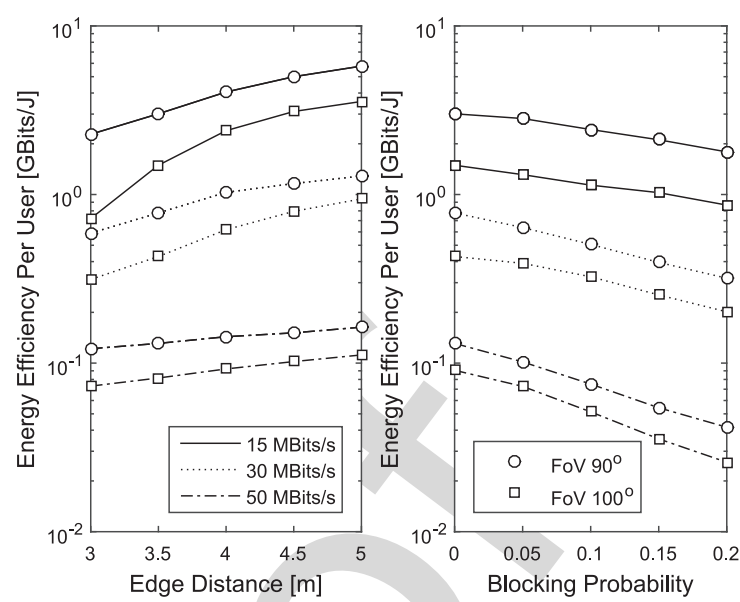

Fig. 4. Energy efficiency per user for edge-distance based A-Cells as a function of the predefined edge-distance threshold (left) and of the LoS and reflectedpath blocking probability (right) using the parameters of Table I.

\section{B. Details}

We now provide detailed observations regarding the edge- 746 distance based A-Cells. In these investigations, we use our 747 baseline algorithm for optimisation. Since similar trends may 748 also be found for the centroid-distance based A-Cells, we omit 749 them for space economy.

1) Effect of Edge Distance: The left subplot of Fig. 4 shows 751 the energy efficiency per user for edge-distance based A-Cells 752 as a function of the predefined edge-distance threshold. It can 753 be seen from the left subplot of Fig. 4 that as expected, guaran- 754 teeing a higher per-user throughput incurs an energy efficiency 755 loss compared to requiring a lower per-user throughput for all 756 predefined edge-distance threshold settings and for both FoVs, 757 demonstrating that any throughput improvement requires extra 758 power to be invested ${ }^{8}$. Furthermore, for all throughput con- 759 straints and for all predefined edge-distance threshold settings, 760 having a higher FoV results in a consistently lower energy 761 efficiency per user than that of a lower FoV, since more inter- 762 ference is encountered and more APs are involved when having 763 a higher FoV. Finally, we observe that the higher the edge- 764 distance threshold, the higher the energy efficiency per user 765 becomes for all throughput constraints and for both FoVs. 766 However, at this stage, we are reluctant to claim the supe- 767 riority of setting towards higher edge-distance threshold for 768 the reasons discussed as follows. In principle, setting a lower 769 edge-distance threshold results into several decoupled A-Cells, 770 whilst having a higher edge-distance threshold results into a 771 few large A-Cells. In both settings, the total number of par- 772 ticipated APs remains similar under a given FoV. Having a 773 few large A-Cells creates a large-dimensional multi-user MISO 774 system. Hence, it is capable of more easily satisfying a given 775 throughput constraint than forming several decoupled A-Cells. 776 However, having a large-dimensional multi-user MISO system 777 will potentially incur additional signal processing costs, such as 778

\footnotetext{
${ }^{8}$ Different modulations incur different levels of energy investments. For example, Color Shift Keying (CSK) relies on the LEDs' color mapping capability, instead of higher DC power, for achieving an increased data rate.
} 
the inversion of a large matrix at the distributed APs as required by the ZF based TPC. Also, sharing data amongst APs of large A-Cells may require more capable back-haul. Hence, network power consumption by additionally considering the signal processing costs and back-haul power consumption is required to determine the most appropriate edge-distance threshold.

2) Effect of Blocking: The right subplot of Fig. 4 shows the energy efficiency per user for edge-distance based A-Cells associated with $d_{0}=3.5 \mathrm{~m}$ as a function of the $\operatorname{LoS}$ and reflected-path blocking probability. It can be seen from the right subplot of Fig. 4 that as expected, the higher the blocking probability, the lower the energy efficiency per user becomes for all throughput constraints and for both FoVs. Furthermore, for all blocking probabilities and for both FoVs, the energy efficiency per user is higher for a lower throughput constraint than for a higher throughput constraint. We observe also that, the slope of the energy efficiency reduction per user is higher for a higher throughput constraint for both FoVs. Finally, for all throughput constraints and for all blocking probabilities, having a higher FoV results in a consistently lower energy efficiency per user than that of a lower FoV, since more interference is encountered and more APs are involved when having a higher FoV. Note that, the blocking model considered here is independent of the FoVs, while we will consider a more realistic FoV-related blocking in the future.

3) Effect of Imperfect Channel Knowledge: The left of Fig. 5 shows the energy efficiency per user for edge-distance based A-Cells associated with $d_{0}=3.5 \mathrm{~m}$ as a function of the error variance of imperfect channel knowledge at the distributed APs used for VT or CT. We assumed Gaussian distributed errors and as expected, the achievable energy efficiency per user degrades upon increasing the error variance for all throughput constraints and for both FoVs. Furthermore, for all error variances and for both FoVs, the energy efficiency per user is higher for a lower throughput constraint than for a higher throughput constraint. Furthermore, for all throughput constraints and for all error variances, having a higher FoV results in a consistently lower energy efficiency per user than that of a lower FoV. These investigations imply the importance of having an accurate channel knowledge, where a modest reduction may be observed in the left of Fig. 5 at an error variance of 0.2 for all throughput constraints and for both FoVs. Hence, a classic trade-off arises between reducing the error variance and investing extra cost, which will be set aside for our future work.

4) Effect of User Density: The right subplot of Fig. 5 shows the energy efficiency per user for edge-distance based A-Cells associated with $d_{0}=3.5 \mathrm{~m}$ as a function of user density. It can be seen from the right subplot of Fig. 5 that as expected for all throughput constraints and for both FoVs, the higher the number of users, the lower the energy efficiency per user becomes, since the interference becomes more pervasive and more number of APs are involved. Importantly, the most substantial drop appears upon increasing the number of users from 10 to 20, followed by a less dramatic energy efficiency erosion per user beyond 20 users. Furthermore, for all user density settings and for both FoVs, the energy efficiency per user is higher for a lower throughput constraint than for a higher throughput constraint. Finally, for all throughput constraints and for all user

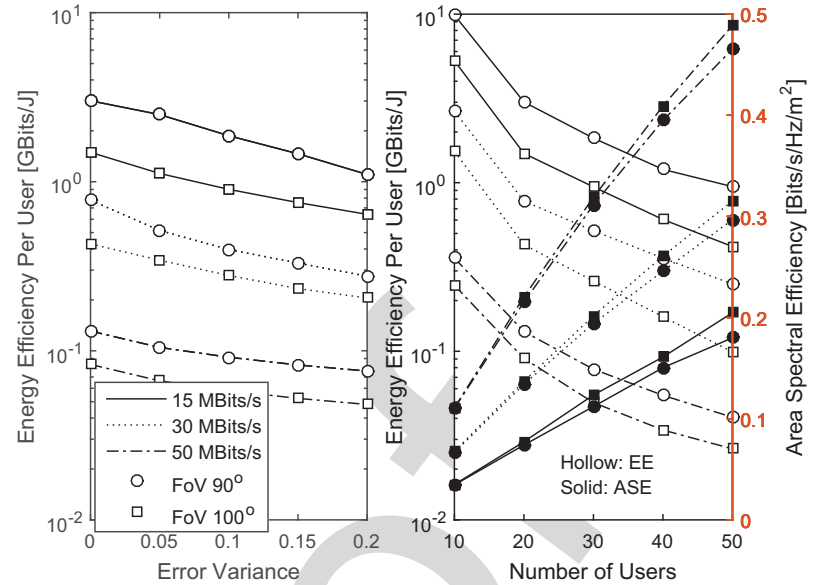

Fig. 5. Energy efficiency per user for edge-distance based A-Cells associated with $d_{0}=3.5 \mathrm{~m}$ as a function of error variance (left) and energy efficiency per user versus area spectral efficiency trade-offs (right) as a function of user density using the parameters of Table I.

density settings, having a higher FoV results in a consistently 837 lower energy efficiency per user than that of a lower FoV, since 838 more interference is encountered and more APs are involved 839 when having a higher FoV.

5) Trade-Offs: The right subplot of Fig. 5 also shows the 841 Area Spectral Efficiency (ASE) for edge-distance based A- 842 Cells associated with $d_{0}=3.5 \mathrm{~m}$ as a function of user density. It 843 can be seen from the right subplot of Fig. 5 that as expected, for 844 all user density settings and for both FoVs, the ASE is higher 845 for a higher throughput constraint than for a lower throughput 846 constraint. Furthermore, for all throughput constraints and for 847 both FoVs, the higher the number of users, the higher the ASE 848 becomes. This is because the aggregated throughput is higher 849 for a higher number of users. However, the ASE improvement 850 seen in the right subplot of Fig. 5 is achieved at the cost of 851 sacrificing the energy efficiency per user. Achieving a higher 852 ASE at the cost of reducing the energy efficiency contradicts 853 to our original design objective. Similarly, for all throughput 854 constraints and for all user density settings, having a higher 855 FoV results in a slightly higher energy efficiency per user than 856 that of a lower FoV, again at the cost of sacrificing the energy 857 efficiency. Finally, we note that the ASE recorded in the right 858 subplot of Fig. 5 is not the maximum achievable ASE, since it 859 was evaluated under the specific constraint of the power alloca- 860 tion strategy obtained when using the energy efficiency as our 861 design objective.

\section{Algorithms}

We now discuss the performance of our proposed algorithm, 864 where we set the minimum per-user throughput constraint to 865 $R=15 \mathrm{MBits} / \mathrm{s}$ and $\mathrm{FoV}$ of $90^{\circ}$ for our investigations. 866

1) Comparison: The left subplot of Fig. 6 shows the energy 867 efficiency per user for edge-distance based A-Cells as a func- 868 tion of the predefined edge-distance threshold, when using 869 both the baseline algorithm and the proposed algorithm with 870 and without adjustments, as detailed in Section II-C3. For our 871 proposed algorithm relying on adjustments, we set $\left|\mathcal{K}_{c}\right|-1872$ and $\left|\mathcal{N}_{c}\right|-1$ as the maximum number of iterations for our 873 

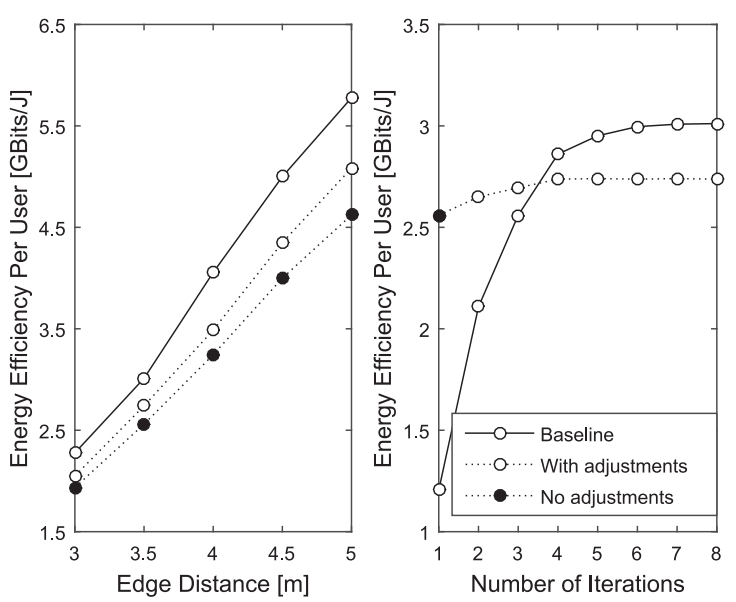

Fig. 6. Energy efficiency per user for edge-distance based A-Cells as a function of the predefined edge-distance threshold (left) and of the number of iterations (right) when using both the baseline algorithm and the proposed algorithm with and without adjustments and using the parameters of Table I.

VT and CT-aided A-Cells, respectively. It can be seen from the left subplot of Fig. 6 that the proposed algorithm operating without adjustments is capable of achieving much of the energy efficiency per user obtained by the baseline algorithm. When relying on additional adjustments, the proposed algorithm achieves a higher energy efficiency per user than that of its non-adjusted counterparts. In particular, it performs quite similarly to the baseline algorithm, when the predefined edge-distance threshold is small.

2) Convergence: The right subplot of Fig. 6 shows the convergence of both the baseline and of the proposed algorithm for edge-distance based A-Cells associated with $d_{0}=3.5 \mathrm{~m}$. It can be seen that the baseline algorithm converges within 6 iterations with further embedded iterations owing to the usage of the dual-decomposition method. For our proposed algorithm, 3 iterations are typically sufficient to approach convergence dispensing with the dual-decomposition method. Despite the ultimate sub-optimality, the intermediate energy efficiency per user achieved by our proposed algorithm is much higher than that of the baseline algorithm. This becomes especially prominent, when we only use a single iteration, which is marked by the solid circle. In the right subplot of Fig. 6, quantitatively, the achievable energy efficiency per user of our proposed algorithm after 3 iterations accounts for slightly more than $90 \%$ that of its ultimate counterpart constituted by our baseline algorithm.

\section{CONCLUSION}

In this paper, we discussed the hitherto-unexplored amorphous structure for constructing energy efficient VLC systems. This problem was approached by the joint design of three inter-linked aspects under the critical consideration of optical constraints. Our numerical results demonstrated that the amorphous structure proposed is beneficial in VLC system design, since it results in a higher energy efficiency than that of the conventional structure. Furthermore, our proposed algorithm is capable of performing close to the baseline algorithm, making it an attractive design alternative. Finally, our proposed amorphous structure constitutes a promising LiFi solution, providing services in various indoor applications, including museums, offices and hospitals. These observations stimulate a range of 912 further research topics, such as the energy efficient VLC system 913 design taking into account the clipping-distortion of DCO- 914 OFDM, the co-design of energy efficient communication and 915 illumination, the network power consumption of VLC system, 916 the comparison to other OOFDM schemes [37]-[41], the inves- 917 tigation of non-linear VT, and finally the robust design under 918 channel estimation uncertainties, etc.

\section{REFERENCES}

[1] G. Li et al., "Energy-efficient wireless communications: Tutorial, survey, 921 and open issues," IEEE Wireless Commun., vol. 18, no. 6, pp. 28-35, Dec. 922 2011.

[2] G. Miao, N. Himayat, and G. Li, "Energy-efficient link adaptation in 924 frequency-selective channels," IEEE Trans. Commun., vol. 58, no. 2, 925 pp. 545-554, Feb. 2010.

[3] D. Ng, E. Lo, and R. Schober, "Energy-efficient resource allocation in 927 OFDMA systems with large numbers of base station antennas," IEEE 928 Trans. Wireless Commun., vol. 11, no. 9, pp. 3292-3304, Sep. 2012.

[4] S. He, Y. Huang, L. Yang, and B. Ottersten, "Coordinated multicell mul- 930 tiuser precoding for maximizing weighted sum energy efficiency," IEEE 931 Trans. Signal Process., vol. 62, no. 3, pp. 741-751, Feb. 2014.

[5] C. He, B. Sheng, P. Zhu, X. You, and G. Li, "Energy- and spectral- 933 efficiency tradeoff for distributed antenna systems with proportional 934 fairness," IEEE J. Sel. Areas Commun., vol. 31, no. 5, pp. 894-902, May 935 2013.

[6] L. B. Le, D. Niyato, E. Hossain, D. I. Kim, and D. T. Hoang, "QoS-aware 937 and energy-efficient resource management in OFDMA femtocells," IEEE 938 Trans. Wireless Commun., vol. 12, no. 1, pp. 180-194, Jan. 2013.

[7] S. Rangan, T. Rappaport, and E. Erkip, "Millimeter-wave cellular wire- 940 less networks: Potentials and challenges," Proc. IEEE, vol. 102, no. 3, 941 pp. 366-385, Mar. 2014.

[8] L. Hanzo, H. Haas, S. Imre, D. O’Brien, M. Rupp, and L. Gyongyosi, 943 "Wireless myths, realities, and futures: From $3 \mathrm{G} / 4 \mathrm{G}$ to optical and 944 quantum wireless," Proc. IEEE, vol. 100, no. Special Centennial Issue, 945 pp. 1853-1888, May 2012.

[9] E. Bjornson, L. Sanguinetti, J. Hoydis, and M. Debbah, “Optimal design 947 of energy-efficient multi-user MIMO systems: Is massive MIMO the 948 answer?," IEEE Trans. Wireless Commun., vol. 14, no. 6, pp. 3059-3075, 949 Jun. 2015

[10] R. Zhang, L.-L. Yang, and L. Hanzo, "Energy pattern aided simultaneous 951 wireless information and power transfer," IEEE J. Sel. Areas Commun., 952 vol. 33, no. 8, pp. 1492-1504, Aug. 2015.

[11] R. Zhang, R. Maunder, and L. Hanzo, "Wireless information and 954 power transfer: From scientific hypothesis to engineering practice," IEEE 955 Commun. Mag., vol. 53, no. 8, pp. 99-105, Aug. 2015.

[12] R. Zhang, J. Wang, Z. Wang, Z. Xu, C. Zhao, and L. Hanzo, "Visible 957 light communications in heterogeneous networks: Pave the way for user- 958 centric design," IEEE Wireless Commun., vol. 22, no. 2, pp. 8-16, Apr. 959 2015.

[13] T. Komine and M. Nakagawa, "Fundamental analysis for visible- 961 light communication system using LED lights," IEEE Trans. Consum. 962 Electron., vol. 50, no. 1, pp. 100-107, Feb. 2004.

[14] J. Grubor, S. Randel, K.-D. Langer, and J. Walewski, "Broadband information broadcasting using LED-based interior lighting," J. Lightw. Technol., vol. 26, no. 24, pp. 3883-3892, Dec. 2008.

[15] R. Zhang and L. Hanzo, "Multi-layer modulation for intensity-modulated 967 direct-detection optical OFDM," J. Opt. Commun. Netw., vol. 5, no. 12, 968 pp. 1402-1412, Dec. 2013.

[16] J. Armstrong, "OFDM for optical communications," J. Lightw. Technol., 970 vol. 27, no. 3, pp. 189-204, Feb. 2009.

[17] A. Azhar, T Tran, and D. O'Brien "A Gigabit/s indoor wireless transmis- 972 sion using MIMO-OFDM visible-light communications," IEEE Photon. 973 Technol. Lett., vol. 25, no. 2, pp. 171-174, Jan. 2013.

[18] D. Tsonev et al., "A 3-Gb/s single-LED OFDM-based wireless VLC link 975 using a gallium nitride $\mu$ LED," IEEE Photon. Technol. Lett., vol. 26, 976 no. 7, pp. 637-640, Apr. 2014.

[19] H. Burchardt, N. Serafimovski, D. Tsonev, S. Videv, and H. Haas, "VLC: 978 Beyond point-to-point communication," IEEE Commun. Mag., vol. 52, 979 no. 7, pp. 98-105, Jul. 2014.

[20] B. Ghimire and H. Haas, "Self-organising interference coordination in optical wireless networks," EURASIP J. Wireless Commun. Netw., vol. 2012 , no. $1,2012$.

\section{.}


[21] D. Bykhovsky and S. Arnon, "Multiple access resource allocation in visible light communication systems," J. Lightw. Technol., vol. 32, no. 8, pp. 1594-1600, Apr. 2014.

[22] M. Biagi, S. Pergoloni, and A. M. Vegni, "LAST: A framework to localize, access, schedule, and transmit in indoor VLC systems," J. Lightw. Technol., vol. 33, no. 9, pp. 1872-1887, May 2015.

[23] F. Jin, R. Zhang, and L. Hanzo, "Resource allocation under delayguarantee constraints for heterogeneous visible-light and RF femtocell," IEEE Trans. Wireless Commun., vol. 14, no. 2, pp. 1020-1034, Feb. 2015.

[24] X. Li, R. Zhang, and L. Hanzo, "Cooperative load balancing in hybrid visible light communications and WiFi," IEEE Trans. Commun., vol. 63, no. 4, pp. 1319-1329, Apr. 2015.

[25] K. Lee and H. Park, "Modulations for visible light communications with dimming control," IEEE Photon. Technol. Lett., vol. 23, no. 16, pp. 1136 1138, Aug. 2011.

[26] I. Din and H. Kim, "Energy-efficient brightness control and data transmission for visible light communication," IEEE Photon. Technol. Lett., vol. 26, no. 8, pp. 781-784, Apr. 2014

[27] S. Kim and S.-Y. Jung, "Modified Reed Muller coding scheme made from the bent function for dimmable visible light communications," IEEE Photon. Technol. Lett., vol. 25, no. 1, pp. 11-13, Jan. 2013.

[28] Y. Suh, C.-H. Ahn, and J. K. Kwon, "Dual-codeword allocation scheme for dimmable visible light communications," IEEE Photon. Technol. Lett., vol. 25, no. 13, pp. 1274-1277, Jul. 2013.

[29] R. Razavi and H. Claussen, "Urban small cell deployments: Impact on the network energy consumption," in Proc. IEEE Wireless Commun. Netw. Conf. Workshops, Apr. 2012, pp. 47-52.

[30] S. Dimitrov, S. Sinanovic, and H. Haas, "Clipping noise in OFDM-based optical wireless communication systems," IEEE Trans. Commun., vol. 60, no. 4, pp. 1072-1081, Apr. 2012.

[31] A. Farid and S. Hranilovic, "Capacity bounds for wireless optical intensity channels with gaussian noise," IEEE Trans. Inf. Theory, vol. 56, no. 12, pp. 6066-6077, Dec. 2010.

[32] X. Li, R. Mardling, and J. Armstrong, "Channel capacity of IM/DD optical communication systems and of ACO-OFDM," in Proc. IEEE Int. Conf. Commun., Jun. 2007, pp. 2128-2133.

[33] S. Dimitrov and H. Haas, "Information rate of OFDM-based optical wireless communication systems with nonlinear distortion," J. Lightw. Technol., vol. 31, no. 6, pp. 918-929, Mar. 2013.

[34] M. Costa, "Writing on dirty paper," IEEE Trans. Inf. Theory, vol. 29, no. 3, pp. 439-441, May 1983.

[35] J. Lee and N. Jindal, "High SNR analysis for MIMO broadcast channels: Dirty paper coding versus linear precoding," IEEE Trans. Inf. Theory, vol. 53, no. 12, pp. 4787-4792, Dec. 2007.

[36] R. Zhang and L. Hanzo, "Cooperative downlink multicell preprocessing relying on reduced-rate back-haul data exchange," IEEE Trans. Veh. Technol., vol. 60, no. 2, pp. 539-545, Feb. 2011

[37] Z. Wang, Q. Wang, S. Chen, and L. Hanzo, "An adaptive scaling and biasing scheme for OFDM-based visible light communication systems," Opt. Exp., vol. 22, no. 10, pp. 12 707-12 715, May 2014.

[38] W. Dinkelbach, "On nonlinear fractional programming," Manage. Sci., vol. 13, pp. 492-498, Mar. 1967.

[39] S. Boyd and L. Vandenberghe, Convex Optimization. Cambridge, U.K.: Cambridge Univ. Press, 2004.

[40] L. Chen, B. Krongold, and J. Evans, "Successive decoding of antiperiodic OFDM signals in IM/DD optical channel," in Proc. IEEE Int. Conf. Commun., May 2010, pp. 1-6.

[41] Q. Gao, C. Gong, S. Li, and Z. Xu, "DC-informative modulation for visible light communications under lighting constraints," IEEE Wireless Commun., vol. 22, no. 2, pp. 54-60, Apr. 2015.

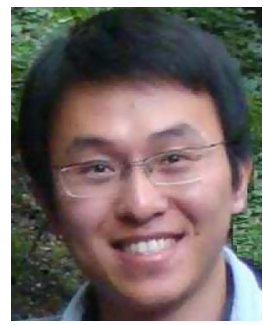

Rong Zhang (M'09) received the Ph.D. degree in wireless communications from University of Southampton (UoS), Southampton, U.K., in 2009. He is an Assistant Professor with Southampton Wireless Group, School of Electronics and Computer Science, UoS. He was with UoS, as a Research Assistant during that period with Mobile Virtual Centre of Excellence, one of UK's largest industrial-academic partnership in ICT. During his postdoctoral period at ECS, he contributed as the UoS Lead Researcher on a number of international projects. After that, he took his industrial consulting leave for Huawei EU R\&D as a System Algorithms Expert. He is also a Guest Researcher of the Centre in Next Generation Computational Modelling, UoS. He has a total of 70+ IEEE/OSA publications, including $40+$ journals (20+ of which as first author). He regularly serves as a Reviewer for the IEEE/OSA journals and has been several times a TPC
member/Invited Session Chair of major conferences. He is a member of the 1060 IET and is the recipient of joint funding from MVCE and EPSRC as well 1061 as the recipient of Worldwide University Network Grant. Owing to his out- 1062 standing academic achievements, he was the recipient of the Prestigious Dean's 1063 Publication Award.

1064

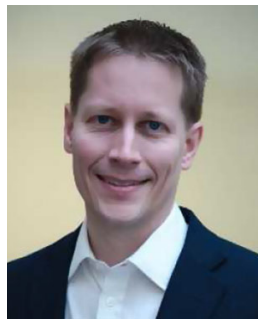

Holger Claussen (S'02-M'04-SM'10) received the 1065 $\mathrm{Ph} . \mathrm{D}$. degree in signal processing for digital com- 1066 munications from the University of Edinburgh, 1067 Edinburgh, U.K., in 2004. He is Leader of Small Cells 1068 Research, Bell Labs, Alcatel-Lucent. In this role, he 1069 and his team are innovating in all areas related to 1070 future evolution, deployment, and operation of small 1071 cell networks to enable exponential growth in mobile 1072 data traffic. His research in this domain has been com- 1073 mercialized in Alcatel-Lucent's Small Cell product 1074 portfolio and continues to have significant impact. 1075 Prior to this, he was the Head of the Autonomous Networks and Systems 1076 Research Department, Bell Labs Ireland, where he directed research in the area 1077 of self-managing networks to enable the first large scale femtocell deployments 1078 from 2009 onwards. He joined Bell Labs in 2004. His research interests include 1079 network optimization, cellular architectures, and improving energy efficiency 1080 of networks. He is the author of more than 90 publications and 110 filed patent 1081 applications. He is a Fellow of the World Technology Network and member 1082 of the IET. He was the recipient of the 2014 World Technology Award in the 1083 individual category Communications Technologies for innovative work of the 1084 greatest likely long-term significance.

1085

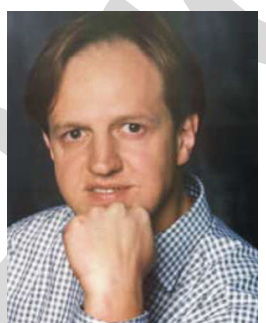

Harald Haas (S'98-M'03) holds the Chair for 1086 Mobile Communications, School of Engineering, 1087 and is the Director of the Li-Fi Research and 1088 Development Centre. He has been working in wire- 1089 less communications for 20 years and held several 1090 posts in industry. He was an invited speaker at TED 1091 Global in 2011, where he demonstrated and coined 1092 LiFi. Li-Fi was listed among the 50 best inventions 1093 in TIME Magazine 2011. Moreover, his work has 1094 been covered in other international media such as the 1095 New York Times, BBC, MSNBC, CNN International, 1096 Wired U.K., and many more. He is an Initiator, a Co-Founder, and the Chief 1097 Scientific Officer (CSO) of pureLiFi Ltd. He holds 31 patents and has more 1098 than 30 pending patent applications. He has authored 300 conference and jour- 1099 nal papers including a paper in Science Magazine. He published two textbooks 1100 with Cambridge University Press. His h-index is 43 (Google). He is CI of pro- 1101 gramme grant TOUCAN (EP/L020009/1), and CI of SERAN (EP/L026147/1). 1102 He currently holds an EPSRC Established Career Fellowship (EP/K008757/1). 1103 In 2014, he was selected as one of ten EPSRC RISE Campaign Leaders. In 1104 2015, he was corecipient of three best paper awards including the IEEE Jack 1105 Neubauer Memorial Award.

1106

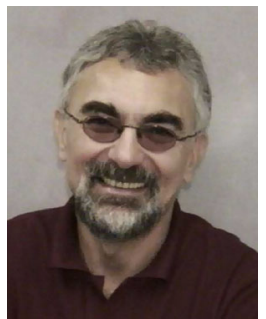

Lajos Hanzo (M'91-SM'92-F'04) received the 1107 degree in electronics, in 1976, the doctorate degree, 1108 in 1983, and the D.Sc. degree. In 2009, he was 1109 awarded the honorary doctorate "Doctor Honoris 1110 Causa" by the Technical University of Budapest. 1111 During his 38-year career in telecommunications, 1112 he has held various research and academic posts in 1113 Hungary, Germany and the U.K. Since 1986, he has 1114 been with the School of Electronics and Computer 1115 Science, University of Southampton, Southampton, 1116 U.K., where he holds the Chair in telecommunica- 1117 tions. He has successfully supervised 80+ Ph.D. students, coauthored 20 John 1118 Wiley/IEEE Press books on mobile radio communications totalling in excess of 1119 10000 pages, published $1400+$ research entries at IEEE Xplore, acted both as 1120 a TPC and the General Chair of the IEEE conferences, presented keynote lec- 1121 tures, and has been awarded a number of distinctions. Currently, he is directing 1122 a 100 -strong academic research team, working on a range of research projects 1123 in the field of wireless multimedia communications sponsored by industry, 1124 the Engineering and Physical Sciences Research Council (EPSRC) U.K., the 1125 European Research Council's Advanced Fellow Grant, and the Royal Society's 1126 Wolfson Research Merit Award. He is an enthusiastic supporter of industrial 1127 and academic liaison and offers a range of industrial courses. He is also a 1128 Governor of the IEEE VTS. From 2008 to 2012, he was the Editor-in-Chief 1129 of the IEEE Press and a Chaired Professor also at Tsinghua University, Beijing, 1130 China. His research is funded by the European Research Council's Senior 1131 Research Fellow Grant. He has $20000+$ citations. He is a Fellow of REng, IET, 1132 and EURASIP. 


\section{QUERIES}

Q1: Please be advised that per instructions from the Communications Society this proof was formatted in Times Roman font and therefore some of the fonts will appear different from the fonts in your originally submitted manuscript. For instance, the math calligraphy font may appear different due to usage of the usepackage[mathcal]euscript. We are no longer permitted to use Computer Modern fonts.

Q2: Note that if you require corrections/changes to tables or figures, you must supply the revised files, as these items are not edited for you.

Q3: Please provide page range for Ref. [20]. 


\title{
Energy Efficient Visible Light Communications Relying on Amorphous Cells
}

\author{
Rong Zhang, Member, IEEE, Holger Claussen, Senior Member, IEEE, Harald Haas, Member, IEEE, \\ and Lajos Hanzo, Fellow, IEEE
}

\begin{abstract}
In this paper, we design an energy efficient indoor visible light communications (VLC) system from a radically new perspective based on an amorphous user-to-network association structure. Explicitly, this intriguing problem is approached from three inter-linked perspectives, considering the cell formation, link-level transmission and system-level optimisation, critically appraising the related optical constraints. To elaborate, apart from proposing hitherto unexplored amorphous cells (A-Cells), we employ a powerful amalgam of asymmetrically clipped optical orthogonal frequency division multiplexing (ACO-OFDM) and transmitter pre-coding aided multi-input single-output (MISO) transmission. As far as the overall system-level optimisation is concerned, we propose a low-complexity solution dispensing with the classic Dinkelbach's algorithmic structure. Our numerical study compares a range of different cell formation strategies and investigates diverse design aspects of the proposed A-Cells. Specifically, our results show that the A-Cells proposed are capable of achieving a much higher energy efficiency per user compared to that of the conventional cell formation for a range of practical field of views (FoVs) angles.
\end{abstract}

Index Terms-Energy Efficiency, Optical Wireless, Small Cells, Optical OFDM, Optical MIMO.

\section{INTRODUCTION}

1) Background: Improving the attainable energy efficiency has been one of the salient design objectives of modern wireless communications [1]. In the post-4G era, quantifying energy efficiency became a challenge owing to the emerging Heterogeneous Networks (HetNet) in pursuit of 'green' designs [2]-[6]. With the launch of the global 5G research initiatives, the community expanded its horizon from Radio Frequency (RF) cellular networks both to millimetre wave [7] and to optical wireless concepts [8]. Owing to this paradigm-shift to higher frequencies, the disruptive large-scale Multi-Input Multi-Output (MIMO) architecture has attracted substantial interests, with the goal of further improving the achievable energy efficiency [9]. Apart from the classic perspectives on

Manuscript received April 15, 2015; revised September 7, 2015; accepted December 11, 2015. This work was supported in part by the EPSRC project (EP/N004558/1) and in part by the EU under the Concerto project as well as that of the European Research Council's (ERC) Advanced Fellow Grant. The data from the paper can be obtained from the University of Southampton ePrints research repository: 10.5258/SOTON/385179.

R. Zhang and L. Hanzo are with the Southampton Wireless, School of Electronics and Computer Science, University of Southampton, Southampton SO17 1BJ, U.K. (e-mail: rz@ecs.soton.ac.uk).

H. Claussen is with the Small Cells Research, Bell Laboratories, AlcatelLucent, Dublin 15, Ireland.

H. Haas is with the Li-Fi R\&D Centre, Institute for Digital Communications, University of Edinburgh, Edinburgh EH9 3JL, U.K.

Color versions of one or more of the figures in this paper are available online at http://ieeexplore.ieee.org.

Digital Object Identifier 10.1109/JSAC.2016.2544598 energy efficiency, the novel concepts of wirelessly powered communications [10], [11] are also emerging. Despite all the above advances, there is a paucity of literature on designing energy efficient optical wireless systems, especially indoor Visible Light Communications (VLC) systems.

With the advent of high-power Light Emitting Diodes (LEDs) and high-sensitivity Photo-Diodes (PD), the VLC concept appears to be especially promising in the small-cell family of the $5 \mathrm{G}$ era [12]. By modulating the visible light produced by the LEDs way above the human eye's fusion frequency, the dual goal of communication and illumination can be realised simultaneously. The pioneering implementation of VLC using LEDs was carried out by the Nakagawa laboratory in 2004 [13], which stimulated significant research attention. The link-level data rates of 100s of MBits/s have been reported using state-of-the-art LEDs and photo-detectors [14]. The modulation schemes have evolved from simple pulse based modulation to more sophisticated Asymmetrically Clipped/DC-biased Optical Orthogonal Frequency Division Multiplexing (ACO/DCO-OFDM) [15], [16]. More ambitious GBits/s targets have also been achieved with the aid of optical MIMO techniques [17] and by using advanced LEDs [18]. Apart from these exciting link-level achievements, the systemlevel study of VLC has also been developed for broadening its scope beyond point-to-point applications [19]-[24].

2) Motivation: However, most of the above-mentioned VLC research aimed for increasing the attainable throughput, whilst paying less attention to energy efficiency. In fact, LEDs are primarily used for illumination, where typically a constant DC power is provided to satisfy the illumination requirements and to maintain sufficient forward biasing voltages across the LEDs for communications. Hence, the additional communication function should not perturb the illumination requirements nor should it violate the LEDs physical limits. Desirably, the extra communication-related power consumption invested should also be as low as possible, while maintaining a minimum required Quality of Service (QoS). This is also true when no illumination is required during daytime. Hence, valuable research has been dedicated to link-level energy efficiency focusing on brightness and dimming control with the aid of both modulation-related [25], [26] and coding-related [27], [28] techniques. However, there is no system-level investigation on energy efficient VLC systems supporting multi-users, which may require a radically new design approach.

When considering the attainable system-level energy efficiency of a particular network, the specific structure of associating the users with the network plays a crucial role. As a result, it has limited benefits to optimise the power consumption of
40 41 42 43 
an inefficient user-to-network association structure. Thanks to the flexibility in indoor VLC systems design [12], we advocate an amorphous user-to-network association structure for indoor VLC systems. To elaborate, in conventional structures, the cells are typically formed from a network-centric perspective, without taking into account the users' positions, where the design flow is based on defining a cell constituted by one or more Access Points (APs) and then associating the users with it. In RF cellular systems having hundreds or thousands of random uniformly distributed users, the conventional structure may indeed be applicable. However, when considering an indoor VLC system supporting only a few dozen users, naively applying the conventional structure may become inefficient, since the users are sporadic. Moreover, in VLC systems, the number of APs may be higher than the number of users, creating an ultra dense AP deployment. Hence, we propose Amorphous Cells (A-Cells) from a user-centric point of view by considering the users' positions, where the design flow is based on grouping the users together and then associating the APs with them, resulting in irregular shape cells.

3) Scope: We design an energy efficient indoor VLC system relying on an amorphous structure under practical optical constraints by considering three interlinked design aspects, namely the cell formation, the link-level transmission and the systemlevel power allocation. Logically, the cell formation strategy determines the specific association between the APs and users, while the transmission strategy and the power allocation jointly determine the signal strength and the amount of interference. Explicitly,

- we propose two A-Cells formation techniques, namely the edge-distance and centroid-distance based A-Cells. The beneficial construction of A-Cells constitutes the basis of a structurally energy efficient indoor VLC system;

- we propose a new link-level transmission scheme by amalgamating the ACO-OFDM and our Vector Transmission (VT) and Combined Transmission (CT) based Multiple Input Single Output (MISO) transmission of [12];

- we propose an efficient low-complexity algorithm for maximising the system-level energy efficiency employing the advocated link-level transmission scheme associated with the proposed A-Cells.

Our paper is organised as follows. In Section II-A, we embark on designing the proposed amorphous structure, while in Section II-B, we discuss the transmission schemes employed and finally we perform system optimisation in Section II-C. The achievable performance of our design is characterised in Section III and we close in Section IV.

\section{SySTEM DESCRIPTION}

Consider an indoor VLC environment having $N$ APs uniformly installed on the ceiling, where each AP is constituted by an array of $L$ LEDs pointing vertically downwards. These APs will be used for communicating with $K$ users and at the same time for providing illumination. As discussed before, supporting wireless communications should not violate the main illumination requirements and should obey the LEDs physical limits. Hence, the communications-related power investment 144 should be as low as possible in order to minimise the perturba- 145 tions imposed on the lightening function, while maintaining a 146 minimum required QoS. This leads to communications-related 147 energy efficiency maximisation as

$$
\max _{\mathcal{F}, \mathcal{T}, \mathcal{P}} f_{t}(\mathcal{F}, \mathcal{T}, \mathcal{P}) / f_{p}(\mathcal{F}, \mathcal{T}, \mathcal{P}),
$$

where $f_{t}(\cdot)$ represents the achievable throughput, which is a 149 function of the cell formation strategy $\mathcal{F}$, of the link-level trans- 150Q1 mission strategy $\mathcal{T}$ and of the power allocation strategy $\mathcal{P}$. 151 Furthermore, $f_{p}(\cdot)$ represents the power consumption, which 152 is also a function of $\mathcal{F}, \mathcal{T}$ and $\mathcal{P}$. Since $\mathcal{F}$ and $\mathcal{T}$ are potentially 153 enumerable, we can reformulate $(1)$ given $\mathcal{F}$ and $\mathcal{T}$ as ${ }^{1}$.

$$
\max _{\mathcal{P}} f_{t \mid \mathcal{F}, \mathcal{T}}(\mathcal{P}) / f_{p \mid \mathcal{F}, \mathcal{T}}(\mathcal{P})
$$

To adopt a clear structure, we will elaborate 1) on the cell 155 formation strategy in Section II-A by describing the channel 156 characteristics in Section II-A1, then introducing the moti- 157 vation of A-Cells in Section II-A2 and finally detailing the 158 construction of A-Cells in Section II-A3; 2) on the transmission 159 strategy in Section II-B by introducing ACO-OFDM and MISO 160 transmission in Section II-B1 and Section II-B2 respectively, 161 and then introducing the optical constraints in Section II-B3; 162 3 ) on the power allocation strategy in Section II-C by out- 163 lining our problem formulation, transformation and simpli- 164 fication in Section II-C1, Section II-C2 and Section II-C3, 165 respectively.

\section{A. Amorphous Structure}

Fixing $\mathcal{F}$ in (1) not only reduces the complexity of the prob- 168 lem, but also constitutes a logically appealing arrangement, 169 since cell formation is the pivotal system design stage.

1) Channel Characteristics: Before introducing the cell 171 formation strategy, a brief description of the VLC channel char- 172 acteristics is essential. The optical channel between the $k$ th user 173 and the $n$th AP is constituted by both the direct Line-of-Sight 174 (LoS) component $h_{k, n}^{0}$ and its reflections, but we only consider 175 the first reflection $h_{k, n}^{1}$, since higher-order indirect reflections 176 are typically negligible. Specifically, the LoS component is 177 given by [13]

$$
h_{k, n}^{0}=\frac{\left(m_{L}+1\right) A_{P D}}{2 \pi d^{2}} \cos ^{m_{L}}(\theta) \cos (\psi) f_{o f}(\psi) f_{o c}(\psi),
$$

where the Lambert index $m_{L}=-1 / \log _{2}\left[\cos \left(\phi_{1 / 2}\right)\right]$ depends on the semi-angle $\phi_{1 / 2}$ at half-illumination of the source. $A_{P D}$ is the physical area of the PD receiver, $d$ is the distance between the $k$ th user and the $n$th AP, $\theta$ is the angle of irradiance from the $n$th AP and $\psi$ is the angle of incidence at the $k$ th user. Still referring to $(3), f_{o f}(\psi)$ and $f_{o c}(\psi)$ denote the gain of the optical

\footnotetext{
${ }^{1}$ Re-evaluating (2) upon the change of cell formation and transmission strategy would impose an excessive complexity. Fortunately, indoor VLC systems typically have low-mobility. Hence, updating (2) semi-adaptively, not instantaneously, strikes a comprise, although the optimal updating frequency is application-specific. However, these interesting points are out of our scope.
} 
185 filter and of the optical concentrator employed, respectively. Furthermore, $f_{o c}(\psi)$ can be written as

$$
f_{o c}(\psi)=n_{r}^{2} / \sin ^{2}(\psi), \psi \leq \psi_{F} ; \quad f_{o c}(\psi)=0, \psi>\psi_{F},
$$

where $\psi_{F}$ represents half of the receiver's Field-of-View (FoV) and $n_{r}$ is the refractive index of a lens at a PD receiver. By contrast, the first reflected component is given by [13]

$$
h_{k, n}^{1}=\sum_{v} \sum_{\tau} \frac{\rho_{r} A_{r} d^{2}}{d_{v, \tau, 1}^{2} d_{v, \tau, 2}^{2}} \cos \left(\alpha_{v, \tau}\right) \cos \left(\beta_{v, \tau}\right) h_{k, n}^{0},
$$

where $d_{v, \tau, 1}$ is the distance between the $n$th AP and the $(v, \tau)$ th reflection point, while $d_{v, \tau, 2}$ is the distance between the $(v, \tau)$ th reflection point and the $k$ th user. Furthermore, $\alpha_{v, \tau}$ and $\beta_{v, \tau}$ denote the angle of incidence for the incoming light and the angle of irradiance for the outgoing light at the $(v, \tau)$ th reflection point, having a tiny area of $A_{r}$ and a reflectance factor of $\rho_{r}$. Furthermore, the pair of summations in (5) represent all the reflections from the walls. Finally, the aggregated channel between the $k$ th user and the $n$th AP is given by $h_{k, n}=$ $h_{k, n}^{0}+h_{k, n}^{1}$, where we assumed single-tap channel response. In the following, we use only the LoS component for constructing the A-Cells, but we will use the aggregated channel for the rest of our design.

2) Motivation of A-Cells: Fig. 1 portrays the conventional structure (left) and the amorphous structure (right) for a $15 \mathrm{~m} \times$ $15 \mathrm{~m}$ indoor VLC system having $8 \times 8$ APs (marked by squares) and 20 users (marked by circles) under three typical scenarios (same, more and less number of cells), where the users' positions are drawn from a uniform random distribution, whilst employing the parameters of Table I. Owing to spacelimitations, in Fig. 1 we only show the edge-distance based A-Cells having a predefined distance threshold of $d_{0}=3.5 \mathrm{~m}$.

Conventional cells typically have a fixed shape. For example, we may partition the $15 \mathrm{~m} \times 15 \mathrm{~m}$ indoor environment into four square-shaped cells having $(4 \times 4)=16$ APs per cell, where the users are associated with cells depending on the users' positions relative to the square-shaped boundary amongst the cells. Within each cell, we may switch off the communications function of the specific APs having no LoS links to the users in their vicinity (indicated by hollow small squares), since improving the energy efficiency is our goal. The related examples may be seen in the left of Fig. 1, where we refer to this bench-marker as 'S16'. Similarly, we also have the special bench-marker of 'S1', which represents the scenario of using each AP to create an individual cell and again, the idle mode is used for those APs, which have no LoS links to the users in order to save energy [29].

A common observation concerning the conventional arrangement 'S16' in the left of Fig. 1 is that the resultant cells are all constrained within the four partitioned areas. However, this arrangement may not be the most appropriate. For example, in the southwest cell of Fig. 1a, the 'boundary user' ' $\mathrm{A}$ ' is clearly far from user ' $C$ ' in the same cell, but it is more close to user ' $B$ ' in the neighbouring cell. Hence, there might be a tendency for user ' $A$ ' to separate from user ' $C$ ' and to join user ' $B$ ', as seen in Fig. 1b of A-Cells. This is also true for the 'boundary user' ' $D$ ' in the northeast cell of Fig. 1a, since it is more close to the users

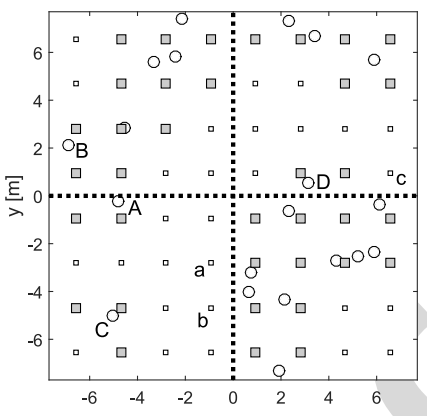

(a) Same number of cells

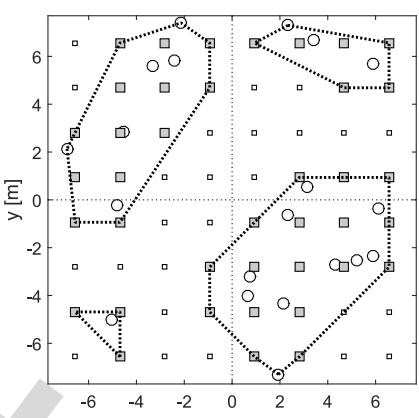

(b) Same number of cells

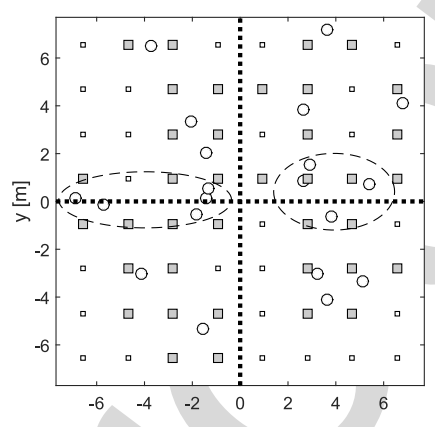

(c) More number of cells

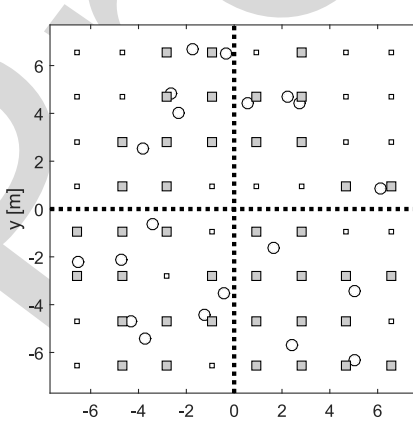

(e) Less number of cells

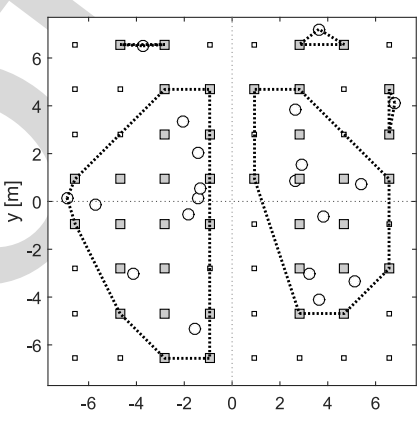

(d) More number of cells

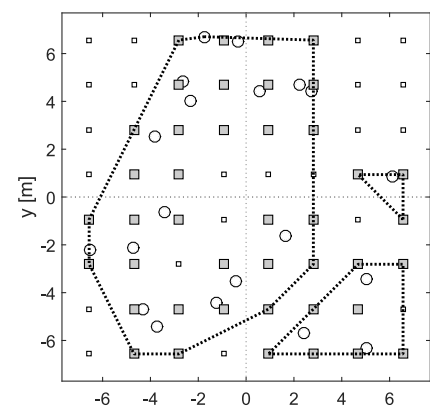

(f) Less number of cells
Fig. 1. Illustration of the conventional (left) and the amorphous structure (right) for VLC indoor systems.

located in the southeast cell, than to the rest of the distant users 237 in the same cell. More examples are shown in Fig. 1c, where 238 two clusters of boundary users highlighted by ellipses join A- 239 Cells of Fig. 1d. In addition to a different user-to-network asso- 240 ciation, the status of APs is also different, where for example, 241 APs ('a', 'b', 'c') were switched from idle mode in conventional 242 cells of Fig. 1a to become active in the A-Cell of Fig. 1b, since 243 they have LoS connections to the associated users. Hence, the 244 proposed A-Cells are capable of breaking boundaries, lead- 245 ing to a higher and a lower number of A-Cells in Fig. 1d 246 and Fig. 1f.

3) Construction of A-Cells: Let us first introduce some 248 common notations. We let $\mathcal{C}$ be the specific set hosting all cells, 249 where for the $c$ th cell $\mathcal{C}_{c}$, we have $\left|\mathcal{N}_{c}\right|$ APs hosted in the set $\mathcal{N}_{c} \quad 250$ serving $\left|\mathcal{K}_{c}\right|$ users of the set $\mathcal{K}_{c}$, with $|\cdot|$ being the cardinality 251 of a set. Note that these notations are in generic sense, includ- 252 ing both conventional cells and A-Cells. We are now ready to 253 discuss the construction of A-Cells.

We firstly construct a full user-to-network association matrix 255 $\boldsymbol{M}^{f}$ having $K$ rows and $N$ columns corresponding to $K$ users 256 
TABLE I

LIST OF PARAMETERS

\begin{tabular}{|l|l|}
\hline \multicolumn{2}{|c|}{ LED-related Parameters } \\
\hline semi-angle at half-illumination $\phi_{1 / 2}$ & $60^{o}$ \\
gain of optical filter $f_{o f}(\psi)$ & 1 \\
physical area for a PD receiver $A_{P D}$ & $1 \mathrm{~cm}^{2}$ \\
O/E conversion factor $\gamma$ & $0.53 \mathrm{~A} / \mathrm{W}$ \\
refractive index $n_{r}$ & 1.5 \\
half of the receiver's FoV $\psi_{F}$ & $45^{\circ}$ \\
Vishay TSHG8200 LED min optical power $P_{\text {min }}$ & $5 \mathrm{~mW}$ \\
Vishay TSHG8200 LED max optical power $P_{\text {max }}$ & $50 \mathrm{~mW}$ \\
optical power to luminous flux conversion factor $\delta$ & $2.1 \mathrm{~mW} / \mathrm{lm}$ \\
\hline \hline \multicolumn{2}{|c|}{ Environment-related Parameters } \\
\hline room size & $15 \times 15 \times 3 \mathrm{~m}^{3}$ \\
user height & $0.85 \mathrm{~m}$ \\
number of users (change in Fig 5) & $20(\mathrm{uniform})$ \\
AP height & $2.5 \mathrm{~m}$ \\
number of APs & $8 \times 8(\mathrm{uniform})$ \\
LED array per AP & $15 \times 15$ \\
reflection efficiency $\rho$ & 0.75 \\
illumination requirement [J $\mathcal{J}_{\text {min }}, \mathcal{J}_{\text {max }}, \mathcal{J}_{\text {avg }}$ ] & {$[200,800,600]$} \\
modulation bandwidth $B$ & $20 \mathrm{MHz}$ \\
blocking probability $p$ (change in Fig 4) & 0 \\
\hline \hline \multicolumn{2}{|c}{ Baseline Algorithm-related Parameters } \\
\hline maximum iterations $\varsigma$ (change in Fig 6) & 20 \\
convergence threshold $\zeta$ & 0.01 \\
\hline
\end{tabular}

and $N \mathrm{APs}^{2}$. The $[k, n]$ th entry of $\boldsymbol{M}^{f}$ is set to the LoS channel $h_{k, n}^{0}$. We then carry out $A P$ anchoring as follows:

1) Initialise user-to-network association matrix $\boldsymbol{M}=\boldsymbol{M}^{f}$.

2) Find the best user-AP pair $\left[k^{*}, n^{*}\right]$ having the strongest LoS channel amongst all entries of $\boldsymbol{M}$ and then collect the best user-index $k^{*}$ in $\boldsymbol{k}^{*}$.

3) Set all entries in the $k^{*}$ th row and in the $n^{*}$ th column of $\boldsymbol{M}$ to zero in order to exclude them from further consideration throughout the AP anchoring process.

4) If there are still positive entries in $\boldsymbol{M}$, we repeat this process from Step 2. Otherwise, we output $\boldsymbol{M}$ as the sub-matrix of $\boldsymbol{M}^{f}$ constituted by all rows from $\boldsymbol{k}^{*}$.

The objective of AP anchoring is to have exclusive user-AP pairs ensuring that each of those users in $\boldsymbol{k}^{*}$ will be served at least by its own anchor AP. Those users who have not found their anchor APs will be scheduled during the next anchoring round, however scheduling is beyond our current scope.

Having the user-to-network association matrix $\boldsymbol{M}$, the distance based A-Cells are constructed, where the users are firstly grouped based on a pre-defined distance threshold $d_{0}$ and then we select APs associated with those users ${ }^{3}$ as follows:

1) Introduce the counter $c$, which is initialised as $c=1$.

2) We commence forming cell $\mathcal{C}_{c}$ by recruiting the first user, who has not been included in any cells. Hence, this user will be the only one in the set $\mathcal{K}_{c}$ and along with the

\footnotetext{
${ }^{2}$ The availability of the full user-to-network association matrix rely on the acquisition of channel knowledge at the AP side, which can be readily estimated at the user side and then fed back to the AP at the cost of a modest overhead. This is because the VLC channels are pre-dominantly static and the channel knowledge can be characterised by a single attenuation factor.

${ }^{3}$ When constructing distance based A-Cells, the mutual distances between users are required. After acquiring the channel knowledge, the distances between users and APs can be inferred from (3). As a result, classical positioning may be used for determining the users' positions. Hence, the mutual distances between users can be readily calculated.
}

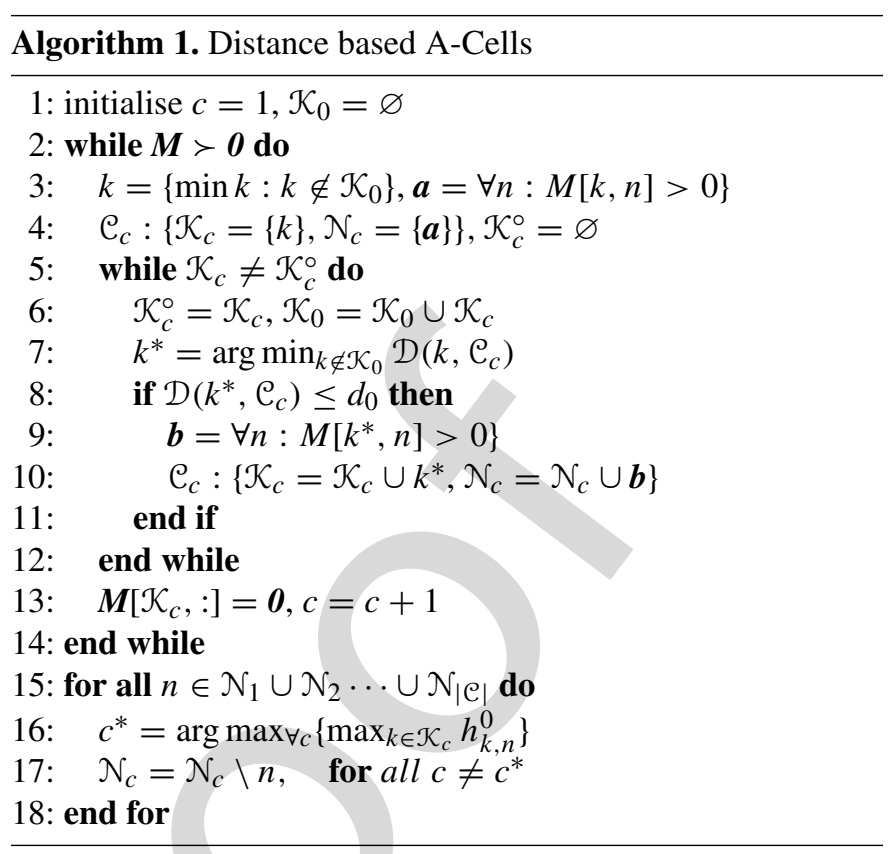

associated AP set $\mathcal{N}_{c}$ containing all the APs providing 282 LoS connections to the first user.

3) Recruit another user from the set of hitherto unas- 284 signed users, who has the smallest distance from the 285 edge/centroid of the cell $\mathfrak{C}_{c}$, provided that their distance is 286 shorter than $d_{0}$. In this step, the edge of the cell is charac- 287 terised by connecting its containing users' positions. We 288 then update cell $\mathfrak{C}_{c}$, which results in the expanded set of 289 $\mathcal{K}_{c}$ and $\mathcal{N}_{c}$.

4) We repeat Step 3 until no additional users can be grouped. 291 As a result, we completed the update of cell $\mathcal{C}_{c}$, which 292 resulted in the final set of $\mathcal{K}_{c}$ and $\mathcal{N}_{c}$.

5) Set all entries of the association matrix $\boldsymbol{M}$ associated with 294 the users in $\mathcal{K}_{c}$ to zero. If there are still positive entries in 295 $\boldsymbol{M}$, we increment $c$ and repeat from Step $2 . \quad 296$ Upon the completion of user grouping, we have to resolve the 297 associated AP ambiguity, since some cells may have conflicting 298 AP assignments. In those cases, any ambiguously assigned AP 299 $n$ is exclusively included in the $c^{*}$ th cell containing those users 300 to whom the ambiguously assigned AP $n$ has the strongest $\operatorname{LoS} 301$ connection. Finally, note that the entire set of APs $\mathcal{N}_{a}$ involved 302 in the ultimately constructed cells, namely $\mathcal{N}_{a}=\mathcal{N}_{1} \cup \mathcal{N}_{2} \cdots \cup 303$ $\mathcal{N}_{|\mathcal{C}|}$, constitutes a subset of whole set of available APs in the 304 room. For those unassigned APs in $\overline{\mathcal{N}}_{a}$, no communications are 305 activated. To ease understanding, we include the pseudo-code in 306 Algorithm 1. Regarding the complexity imposed, the first step 307 of AP anchoring requires only an order of $\mathcal{O}(K)$ operations and 308 an order of $\mathcal{O}(K N \log (K N))$ for finding the best user-AP pair. 309 Furthermore, the complexity of Algorithm 1 is linear within the 310 range of $\left[\mathcal{O}\left(K^{2}\right), \mathcal{O}\left(K^{3}\right)\right]$ owing to its hierarchical algorithmic 311 structure.

\section{B. Link-level Transmission}

Let us now discuss the transmission strategy relying on the 314 amalgamation of ACO-OFDM and MISO transmission. Our 315 
forthcoming elaborations are equally applicable both to the conventional cells and to the proposed A-Cells.

1) ACO-OFDM: We first consider the downlink ACOOFDM transmission from the $n$th AP $n \in \mathcal{N}_{c}$ to the $k$ th user $k \in \mathcal{K}_{c}$ in the $c$ th cell $\mathcal{C}_{c}$. Let $N_{s}$ represent the number of ACO-OFDM sub-channels and let $s_{n} \in \mathbb{C}^{N_{s} / 4}$ represent the Frequency Domain (FD) information-bearing symbol vector transmitted from the $n$th AP. Then the resultant ACO-OFDM symbol vector $s_{n}^{f} \in \mathbb{C}^{N_{s}}$ is constituted by the odd sub-channel entries of zero and the even sub-channel entries of

$$
s_{n}^{f}[m]= \begin{cases}s_{n}[m / 2] & \text { if } m \leq N_{s} / 2 \text { and is even } \\ s_{n}^{\operatorname{conj}^{[}\left[\frac{N_{s}-m+2}{2}\right]} & \text { if } m>N_{s} / 2 \text { and is even. }\end{cases}
$$

It becomes plausible that the ACO-OFDM mapping of (6) obeys the Hermitian symmetry property, which allows us to create real-valued Time Domain (TD) signal samples $\boldsymbol{s}_{n}^{t} \in \mathbb{R}^{N_{s}}$ after the classic IFFT operation. Since the odd-indexed FD subchannels are set to zero in the specific ACO-OFDM mapping of (6), the first-half of the TD signal samples are copied to the second-half of the TD signal samples, albeit with their signs flipped. As a result, the TD signal samples can be conveyed with all the negative parts clipped at zero. The clipping-distortion imposed by the removal of the negative amplitudes only occurs at the odd-indexed FD sub-channels carrying no data and hence can be ignored, despite the fact that the amplitude of the TD signal samples is halved.

The clipped positive and real-valued TD signal samples $\tilde{\boldsymbol{s}}_{n}^{t} \in$ $\mathbb{R}_{+}^{N_{s}}$ in conjunction with the DC-bias current are then forwarded to the LEDs, where the TD signal envelope is used for modulating the intensity of the LEDs. To elaborate a little further, the clipped TD signal samples $\tilde{\boldsymbol{s}}_{n}^{t}$ obey the clipped Gaussian distribution with a parameter of $\sigma_{s_{n}^{t}}$. Hence, the average optical power of the TD signal samples $\tilde{\boldsymbol{s}}_{n}^{t}$ is $P^{\circ}=\sigma_{s_{n}^{t}} / \sqrt{2 \pi}$ and the total radiated optical power plus DC is $P=P^{d c}+P^{\circ}$. Correspondingly, the average electronic power of the TD signal samples $s_{n}^{t}$ is $P_{t}^{e}=\sigma_{s_{n}^{t}}^{2}$, while the average electronic power of the FD ACO-OFDM symbol vector associated with its even-order half is $P_{f}^{e}=2 P_{t}^{e}$.

The $k$ th user's receiver is comprised of a PD and a transimpedance amplifier. After the removal of DC component and followed by direct detection relying on the Optical to Electrical $(\mathrm{O} / \mathrm{E})$ conversion factor of $\gamma$, the discrete-time model of the received FD symbol vector $\boldsymbol{y}_{k} \in \mathbb{C}^{N_{s}}$ after FFT becomes

$$
\boldsymbol{y}_{k}=(\gamma / 2) \operatorname{diag}[\boldsymbol{h}] \boldsymbol{s}_{n}^{f}+\boldsymbol{I}_{k}+\boldsymbol{w}_{k},
$$

where $\boldsymbol{h} \in \mathbb{R}^{N_{s}}$ hosts the FD channel responses. Since the ACO-OFDM VLC channels can be safely considered as being non-dispersive for a bandwidth $B$ upto $20 \mathrm{MHz}$ [30], each entry of $\boldsymbol{h}$ becomes a single-tap gain factor $h_{k, n}{ }^{4}$. Furthermore, $\boldsymbol{I}_{k} \in$ $\mathbb{C}^{N_{s}}$ denotes the interference imposed on the $k$ th user. Finally, $\boldsymbol{w}_{k} \in \mathbb{C}^{N_{s}}$ represents the noise vector accounting for both the shot noise and the thermal noise at the receiver, which can be

\footnotetext{
${ }^{4}$ We consider white LEDs constructed by using blue LEDs having a phosphor layer, which have a typical bandwidth of $20 \mathrm{MHz}$. This is achievable if a blue filter is used at the receiver side. Our methodology is also eminently applicable, when LEDs with $\geq 100 \mathrm{MHz}$ bandwidth become available.
}

modelled as zero-mean complex-valued AWGN with a vari- 363 ance of $\sigma^{2}=N_{0} B$, where $N_{0} \approx 10^{-22} \mathrm{~A}^{2} / \mathrm{Hz}$ [14]. Extracting 364 the information-bearing sub-channels from $\boldsymbol{y}_{k}$, for each $m \in 365$ $\left\{2,4, \ldots, N_{s} / 2\right\}$ we have,

$$
y_{k}[m]=(\gamma / 2) h_{k, n} s_{n}^{f}[m]+I_{k}[m]+w_{k}[m] .
$$

Remark II-B1: Energy efficiency maximisation of conven- 367 tional RF systems typically relies on Shannon's capacity 368 expression for $f_{t}(\cdot)$ in (2), assuming Gaussian-distributed 369 signalling. However, optical systems relying on Intensity 370 Modulation/Direct Detection (IM/DD) can only have real- 371 valued positive signals. Unfortunately, there is a lack of exact 372 capacity expressions for optical systems, despite the existence 373 of various forms of capacity bounds [31]. Hence, we con- 374 sider ACO-OFDM, since it is a widely used optical modulation 375 scheme exhibiting a higher power efficiency than DCO-OFDM, 376 which allows us to derive an analytically tractable capacity 377 expression [32], [33] for our energy efficiency maximisation 378 problem to be discussed in Section II-C. Suffice to say that, the 379 capacity expression of DCO-OFDM has to take into consider- 380 ation the non-linear clipping distortion effects [33], which are 381 hence set aside for our future research. 382

2) MISO Transmission: Having discussed the above point- 383 to-point scenario, let us now discuss the multi-user scenario. 384 For the $c$ th cell having $\left|\mathcal{N}_{c}\right|$ APs and $\left|\mathcal{K}_{c}\right|$ users, the equivalent 385 physical layer may be modelled as a multi-user MISO system 386 for $\left|\mathcal{K}_{c}\right|>1$ or as a single-user MISO system for $\left|\mathcal{K}_{c}\right|=1.387$ In the former case, we employ VT based on Zero Forcing 388 (ZF) Transmit Pre-Coding (TPC) for eliminating the inter-user- 389 interference within the $c$ th cell, while for the latter case, we 390 employ CT for maximising the received signal power. 391

When $\left|\mathcal{K}_{c}\right|=1$, CT is employed, where we transmit the 392 same signal $s_{n}^{f}[m]=x_{k_{0}}[m]$ from all $n \in \mathcal{N}_{c}$ APs to the only 393 user $k_{0} \in \mathcal{K}_{c}$. The signals arriving at the $k_{0}$ th receiver can be 394 constructively combined, since all the channels emerging from 395 all $\mathcal{N}_{c}$ APs to the user $k_{0}$ are positive. Hence, for the $m$ th sub- 396 channel $m \in\left\{2,4, \ldots, N_{s} / 2\right\}$, we have the single-user MISO 397 expression of (8) written as

$$
y_{k_{0}}[m]=(\gamma / 2) \sum_{n \in \mathcal{N}_{c}} h_{k_{0}, n} \varrho_{n}^{c t} x_{k_{0}}[m]+I_{k_{0}}[m]+w_{k_{0}}[m],
$$

where we set normalised electronic power of $x_{k_{0}}[m]$ being unity 399 and let $\varrho_{n}^{c t}=\sqrt{2 P_{c, n}^{c t}}$ with $P_{c, n}^{c t}$ to be optimised. By contrast, 400 for $\left|\mathcal{K}_{c}\right|>1$, VT is employed. For the $m$ th sub-channel $m \in 401$ $\left\{2,4, \ldots, N_{s} / 2\right\}$, we have the multi-user MISO expression of 402 (8) written as

$$
\boldsymbol{y}[m]=(\gamma / 2) \boldsymbol{H s}^{f}[m]+\boldsymbol{I}[m]+\boldsymbol{w}[m],
$$

where $\boldsymbol{y}[m] \in \mathbb{C}^{\left|\mathcal{K}_{c}\right|}$ is the received FD symbol vector of all the 404 $\left|\mathcal{K}_{c}\right|$ users in the $c$ th cell on the $m$ th sub-channel, while $\boldsymbol{s}^{f}[m] \in 405$ $\mathbb{C}^{\left|\mathcal{N}_{c}\right|}$ is the FD symbol vector transmitted from all $\left|\mathcal{N}_{c}\right|$ APs in 406 the $c$ th cell on the $m$ th sub-channel. Finally, $\boldsymbol{H} \in \mathbb{R}^{\left|\mathcal{K}_{c}\right| \times\left|\mathcal{N}_{c}\right|} 407$ is the channel between the $\left|\mathcal{K}_{c}\right|$ users and the $\left|\mathcal{N}_{c}\right|$ APs, while 408 $\boldsymbol{I}[m] \in \mathbb{C}^{\left|\mathcal{K}_{c}\right|}$ is the inter-cell-interference imposed on all $\left|\mathcal{K}_{c}\right| \quad 409$ users in the $c$ th cell on the $m$ th sub-channel. To eliminate the 410 inter-user-interference, we employ TPC, which is formulated 411 
as $\boldsymbol{s}^{f}[m]=\boldsymbol{G} \boldsymbol{x}[m]$, where $\boldsymbol{x}[m] \in \mathbb{C}^{\left|\mathcal{K}_{c}\right|}$ is the multi-user FD symbol vector and the ZF precoding $\boldsymbol{G} \in \mathbb{R}^{\left|\mathcal{N}_{c}\right| \times\left|\mathcal{K}_{c}\right|}$ is explicitly formulated as $\boldsymbol{G}=\boldsymbol{H}^{\dagger}$, which is the pseudo-inverse of $\boldsymbol{H}$. Hence, (10) can be decomposed into $\left|\mathcal{K}_{c}\right|$ parallel streams and for any $k \in \mathcal{K}_{c}$, yielding

$$
y_{k}[m]=(\gamma / 2) \varrho_{k}^{v t} x_{k}[m]+I_{k}[m]+w_{k}[m],
$$

where we set normalised electronic power of $x_{k}[m]$ being unity and let $\varrho_{k}^{v t}=\sqrt{2 P_{c, k}^{v t}}$ with $P_{c, k}^{v t}$ to be optimised.

Remark II-B2: It is natural to consider multi-user MISO transmission in VLC systems using VT to eliminate the interuser-interference. The roots of VT are in the celebrated results of information theory, where Dirty Paper Coding (DPC) is found to be capable of achieving the broadcast channel's capacity [34]. Owing to its non-linear complex nature of implementing DPC, the low-complexity ZF constitutes a popular alternative, which was shown to exhibit a negligible performance loss compared to DPC in the high Signal to Noise Ratio (SNR) regime, when the number of APs is larger than the number of users [35]. Hence, this important theoretical finding benefits directly a range of modern communications systems. For example, the concept of VT is similar to the successful employment of 'vectoring' in the state-of-the-art Digital Subscriber Line (DSL) based G.fast system invoked for coping with the crosstalk between twisted pairs. This operation is also reminiscent of the concept of the Coordinated Multiple Point (CoMP) transmission regime of classic RF cellular communications conceived for mitigating the inter-cell-interference at the cell edge [36]. In practice, to facilitate VT from $\left|\mathcal{N}_{c}\right|$ APs to $\left|\mathcal{K}_{c}\right|$ users, both the channel matrix $\boldsymbol{H}$ and the users' data $\boldsymbol{x}[m]$ have to be shared amongst the $\left|\mathcal{N}_{c}\right|$ APs. Fortunately, this requirement can be satisfied, since the VLC channels are prepredominately static, while the sharing of all users' data $\boldsymbol{x}[\mathrm{m}]$ requires a more capable back-haul.

3) Optical Constraints: Typically, the forward current of the DC-biased and clipped TD signal samples should be within the LED's dynamic range [30]. Since the total radiated optical power is directly proportional to the forward current, we describe the optical constraints in terms of their optical power.

The total optical power radiated from an AP should satisfy the per-LED dynamic range of $P_{\min } \leq P / L \leq P_{\max }$, where we assume that each of the $L$ LEDs constituting an AP emits the same optical power. For example, a practical dynamic range of a Vishay TSHG8200 LED is between $P_{\min }=5 \mathrm{~mW}$ and $P_{\max }=50 \mathrm{~mW}$ at room temperature. For satisfying a predefined illumination requirement constituted by the minimum illumination $\mathcal{J}_{\min }$, the maximum illumination $\mathcal{J}_{\text {max }}$ and the average illumination $\mathcal{J}_{\text {avg }}$, we find the minimum required optical power $P_{\min }^{\text {illu }}$ by solving the problem of

$$
\begin{gathered}
P_{\text {min }}^{\text {illu }}=\min P \quad \text { s.t. } \\
\min _{\mu \in\left[1, K_{p}\right]} \sum_{n=1}^{N} h_{\mu, n}^{\text {illu }} P \geq \mathcal{J}_{\text {min }}, \quad \max _{\mu \in\left[1, K_{p}\right]} \sum_{n=1}^{N} h_{\mu, n}^{\text {illu }} P \leq \mathcal{J}_{\text {max }}, \\
\mathcal{J}_{\text {avg }}^{-} \leq \frac{1}{K_{p}} \sum_{\mu=1}^{K_{p}} \sum_{n=1}^{N} h_{\mu, n}^{\text {illu }} P \leq \mathcal{J}_{\text {avg }}^{+}, \quad P_{\text {min }} \leq P / L \leq P_{\text {max }},
\end{gathered}
$$

We may also define a global energy efficiency as the sum 494 throughput of all cells divided by the total power consump- 495 tion of all cells, where a centralised approach has to be used. 496 By contrast, the per-cell basis definition of (16) supports a 497

\footnotetext{
${ }^{5}$ We fix the DC-biasing by focusing our attention on the communicationsrelated energy efficiency maximisation, despite the possibility of adaptive signal scaling and DC biasing [37]. Note that, when DCO-OFDM is considered, optimising the DC-bias component becomes critical [30].
}

where $\delta$ denotes the optical power to luminous flux conversion 463 factor [14]. Similarly, we also find the maximum optical power 464 $P_{\max }^{i l l u}=\max P$ capable of satisfying the constraints of (12). 465 Note that having predefined illumination requirements also pre- 466 vents the saturation of the PD receiver, hence we assume the 467 absence of any further clipping at the receiver. As a result, 468 LED's physical limits, we have the optical constraint of

In this paper, we fix the DC-bias component ${ }^{5}$ and assume 471 only negative clipping is incurred by our ACO-OFDM scheme, 472 because we can always set an appropriate margin for prevent- 473 ing upper clipping imposed by the high Peak to Average Power 474 Ratio (PAPR) of ACO-OFDM TD signal samples by control- 475 ling the maximum optical output power. More explicitly, to 476 avoid insufficient forward biasing, we set the DC-bias com- 477 ponent to be at least as high as the minimum optical power 478 required for satisfying the LED's dynamic range. On the other 479 hand, we also adjust the DC-bias component to 'just' sat- 480 isfy the predefined illumination requirement. Hence, we have 481 $P^{d c} / L=\max \left\{P_{m i n}^{i l l u}, P_{\min }\right\}$ and the optical requirement of (14) 482 becomes $0 \leq P^{\circ} / L \leq P_{\text {max }}^{\circ}$, where

represents the maximum tolerable additional optical power 484 of each LED, so that the communication function would not 485 violate the illumination and LED instrument requirements.

Let us now discuss the system-level energy efficiency max- 488 imisation. Our forthcoming elaborations are equally applicable 489 both to the conventional cells and to the proposed A-Cells. $\quad 490$

1) Formulation: Let us formulate our energy efficiency 491 maximisation problem by defining system's mean energy effi- 492 ciency on a per-cell basis as

$$
\max _{\mathcal{P}} \mathrm{EE}=\frac{1}{|\mathfrak{C}|} \sum_{\mathrm{c}} \mathrm{EE}_{\mathrm{c}}(\mathcal{P}),
$$
by taking into account both the illumination requirements and 469 
more scalable and efficient distributed approach. Since $|\mathrm{C}|$ is a constant, (16) can be solved equivalently by optimising $\max _{\mathcal{P}} \sum_{c} \mathrm{EE}_{\mathrm{c}}(\mathcal{P})$, explicitly

$$
\max _{\boldsymbol{P}} \sum_{c \in \mathcal{C}_{v t}} \frac{f_{t, c}^{v t}(\boldsymbol{P})}{f_{p, c}^{v t}\left(\boldsymbol{P}_{c}^{v t}\right)}+\sum_{c \in \mathfrak{C}_{c t}} \frac{f_{t, c}^{c t}(\boldsymbol{P})}{f_{p, c}^{c t}\left(\boldsymbol{P}_{c}^{c t}\right)} \quad \text { s.t. } \quad \mathrm{C} 1, \mathrm{C} 2
$$

where $\mathcal{C}_{v t}$ and $\mathcal{C}_{c t}$ are the sets hosting the specific cells that employ VT and CT, respectively. Furthermore, we have $\boldsymbol{P}=$ $\left\{\boldsymbol{P}^{v t} ; \boldsymbol{P}^{c t}\right\}$ acting as the power allocation strategy, which is constituted by that of all VT-aided cells $\boldsymbol{P}^{v t}=\left\{\boldsymbol{P}_{c}^{v t}, \forall c \in \mathcal{C}_{v t}\right\}$ and all CT-aided cells $\boldsymbol{P}^{c t}=\left\{\boldsymbol{P}_{c}^{c t}, \forall c \in \mathcal{C}_{v t}\right\}$, where $\boldsymbol{P}_{c}^{v t}$ and $\boldsymbol{P}_{c}^{c t}$ are the power allocation strategy for the $c$ th cell using VT and CT, respectively. By scrutinising (17), we find that only those cells in $\mathrm{C}_{v t}$ and $\mathrm{C}_{c t}$ are included in our optimisation. This implies that those APs, which are not included in $\mathcal{C}_{v t}$ and $\mathcal{C}_{c t}$, are switched into their idle mode.

Still referring to (17), since ACO-OFDM is employed, we can assume Gaussian signalling for $x_{k}[\mathrm{~m}]$ in (11) and for $x_{k_{0}}[\mathrm{~m}]$ in (9). Hence, we arrive at

$$
\begin{aligned}
& f_{t, c}^{v t}(\boldsymbol{P})=\sum_{k \in \mathcal{K}_{c}} \underbrace{\kappa \log _{2}\left[1+\frac{\left(\gamma^{2} / 2\right) P_{c, k}^{v t}}{\sigma^{2}+I_{c, k}\left(\boldsymbol{P}_{\bar{c}}^{v t}, \boldsymbol{P}^{c t}\right)}\right]}_{f_{t, c, k}^{v t}\left(P_{c, k}^{v t}, \boldsymbol{P}_{\bar{c}}^{v t}, \boldsymbol{P}^{c t}\right)}, \\
& f_{t, c}^{c t}(\boldsymbol{P})=\kappa \log _{2}\left[1+\frac{\left(\gamma^{2} / 2\right)\left(\sum_{n \in \mathcal{N}_{c}} h_{k_{0}, n} \sqrt{P_{c, n}^{c t}}\right)^{2}}{\sigma^{2}+I_{c, k_{0}}\left(\boldsymbol{P}_{\bar{c}}^{c t}, \boldsymbol{P}^{v t}\right)}\right],
\end{aligned}
$$

where $\kappa=(1-p) B / 4$ is a constant, with the factor of 4 representing the bandwidth efficiency loss owing to the employment of ACO-OFDM having a bandwidth of $B$ and $p$ denoting the blocking probability. Furthermore, $I_{c, k}(\cdot)$ denotes the interference imposed on the $k$ th user in the $c$ th VT-aided cell, while $I_{c, k_{0}}(\cdot)$ denotes the interference imposed on the only user $k_{0}$ in the $c$ th CT-aided cell. In (18) and (19), $\boldsymbol{P}_{\bar{c}}^{v t}$ and $\boldsymbol{P}_{\bar{c}}^{c t}$ represent the power allocation excluding the $c$ th cell from all VT-aided and CT-aided cells, respectively, which are used for evaluating the interference terms $I_{c, k}(\cdot)$ and $I_{c, k_{0}}(\cdot)$. Finally, since the DC-bias component is fixed for illumination, we consider the communications-related power consumption in the electronic domain, which may be written as

$$
\begin{aligned}
& f_{p, c}^{v t}\left(\boldsymbol{P}_{c}\right)=\sum_{n \in \mathcal{N}_{c}} \sum_{k \in \mathcal{K}_{c}} g_{n, k}^{2} P_{c, k}^{v t}, \\
& f_{p, c}^{c t}\left(\boldsymbol{P}_{c}\right)=\sum_{n \in \mathcal{N}_{c}} P_{c, n}^{c t},
\end{aligned}
$$

where $g_{n, k}$ is the $[n, k]$ th entry of the TPC matrix $\boldsymbol{G}^{6}$. Furthermore, as far as the optimisation constraints are

\footnotetext{
${ }^{6} \mathrm{We}$ only consider transmission power as our source of power consumption in (20) and (21). In our future work, other sources of power consumption, such as the signal processing costs, back-haul power consumption etc would be desired to paint the whole picture in terms of the entire network power consumption [29]. However, at the time of writing, quantifying the network power consumption for VLC systems remains an open issue, since integrating it with a certain VLC back-haul requires dedicated treatment.
}

concerned, we impose the per-LED optical power constraint 529 pair $\mathrm{C} 1$ of

$$
\begin{aligned}
\frac{1}{L} \sqrt{\sum_{k \in \mathcal{K}_{c}} g_{n, k}^{2} P_{c, k}^{v t} / 2 \pi} & \leq P_{\text {max }}^{\circ} \quad \forall n \in \mathcal{N}_{c}, \forall c \in \mathcal{C}_{v t}, \\
\frac{1}{L} \sqrt{P_{c, n}^{c t} / 2 \pi} & \leq P_{\text {max }}^{\circ} \quad \forall n \in \mathcal{N}_{c}, \forall c \in \mathcal{C}_{c t} .
\end{aligned}
$$

We also impose the per-user throughput constraint pair C2 for 531 guaranteeing a minimum required $\mathrm{QoS}$ as

$$
\begin{aligned}
f_{t, c, k}^{v t}\left(P_{c, k}^{v t}, \boldsymbol{P}_{\bar{c}}^{v t}, \boldsymbol{P}^{c t}\right) & \geq R \quad \forall k \in \mathcal{K}_{c}, \forall c \in \mathcal{C}_{v t}, \\
f_{t, c}^{c t}(\boldsymbol{P}) & \geq R \quad \forall c \in \mathcal{C}_{c t} .
\end{aligned}
$$

Note that neglecting the per-user throughput constraint pair C2 533 by unilaterally maximising the energy efficiency results in an 534 ill-defined problem, since the user QoS target is ignored.

2) Transformation: The objective function defined in (17) 536 is complex due to the coupled nature of the power allocation 537 strategy. Hence, we opt for decoupling the original problem 538 formulated in (17) so as to allow efficient distributed process- 539 ing. More explicitly, instead of evaluating the true interference 540 term of (18) and (19), we consider the interference upper bound 541 by assuming that all other cells transmit at their maximum 542 permissible optical power. This implies that we carry out guar- 543 anteed energy efficiency maximisation. More specifically, we 544 use constant $I_{c, k}\left(P_{\text {max }}^{\circ}\right)$ to represent the maximum possible 545 interference imposed on the $k$ th user in the $c$ th VT-aided cell, 546 which is written as

$$
I_{c, k}\left(P_{\text {max }}^{\circ}\right)=\gamma^{2} \pi \sum_{\tilde{c} \in \mathcal{C}, \tilde{c} \neq c} \sum_{n \in \mathcal{N}_{\tilde{c}}} h_{k, n}^{2}\left(L P_{\text {max }}^{\circ}\right)^{2} .
$$

Similarly, by replacing $k=k_{0}$ in (26), we have $I_{c, k_{0}}\left(P_{\text {max }}^{\circ}\right)$ rep- 548 resenting the maximum possible interference imposed on the 549 only user $k_{0}$ in the $c$ th CT-aided cell. Correspondingly, we can 550 reformulate the lower bound of (18) and (19) as

$$
\begin{aligned}
& f_{t, c}^{v t, l}\left(\boldsymbol{P}_{c}^{v t}\right)=f_{t, c}^{v t}(\boldsymbol{P})_{\left\{I_{c, k}\left(\boldsymbol{P}_{\bar{c}}^{v t}, \boldsymbol{P}^{c t}\right) \mapsto I_{c, k}\left(P_{\max }^{\circ}\right), \forall k \in \mathcal{K}_{c}\right\}}, \\
& f_{t, c}^{c t, l}\left(\boldsymbol{P}_{c}^{c t}\right)=f_{t, c}^{c t}(\boldsymbol{P})_{\left\{I_{c, k_{0}}\left(\boldsymbol{P}_{\bar{c}}^{c t}, \boldsymbol{P}^{v t}\right) \mapsto I_{c, k_{0}}\left(P_{\text {max }}^{\circ}\right)\right\}} \cdot
\end{aligned}
$$

It is plausible that (27) and (28) become only the function of 552 the power allocation strategy of the $c$ th cell, which is decoupled 553 from other cells. Furthermore, since the composition in (19) or 554 in its lower bound expression of (28) results in a non-concave 555 function, we rely on the lower bounding of (28) in order to 556 arrive at the concave formulation of

$$
f_{t, c}^{c t, l l}\left(\boldsymbol{P}_{c}^{c t}\right)=\kappa \log _{2}\left[1+\frac{\left(\gamma^{2} / 2\right) \sum_{n \in \mathcal{N}_{c}} h_{k_{0}, n}^{2} P_{c, n}^{c t}}{\sigma^{2}+I_{c, k_{0}}\left(P_{\text {max }}^{\circ}\right)}\right] .
$$

Upon taking into account (27) and (29), we arrive at the new 558 constraint pair $\mathrm{C} 2$ formulated as

$$
\begin{aligned}
f_{t, c, k}^{v t, l}\left(P_{c, k}^{v t}\right) & \geq R \quad \forall k \in \mathcal{K}_{c}, \forall c \in \mathcal{C}_{v t}, \\
f_{t, c}^{c t, l l}\left(\boldsymbol{P}_{c}^{c t}\right) & \geq R \quad \forall c \in \mathcal{C}_{c t} .
\end{aligned}
$$

Clearly, this new constraint pair is more strict than the origi- 560 nal constraint pair of (24) and (25). Lastly, the constraint pair 561 
C1 remains the same, as in (22) and (23). Following the above transformation, the decoupled energy efficiency maximisation problem becomes

$$
\max _{\boldsymbol{P}} \sum_{c \in \mathcal{C}_{v t}} \frac{f_{t, c}^{v t, l}\left(\boldsymbol{P}_{c}^{v t}\right)}{f_{p, c}^{v t}\left(\boldsymbol{P}_{c}^{c t}\right)}+\sum_{c \in \mathfrak{C}_{c t}} \frac{f_{t, c}^{c t, l l}\left(\boldsymbol{P}_{c}^{c t}\right)}{f_{p, c}^{c t}\left(\boldsymbol{P}_{c}^{c t}\right)}
$$

$$
\text { s.t. (22), (23), (30), (31). }
$$

Hence, the problem formulated in (32) can be solved in form of $|\mathrm{C}|$ parallel sub-problems, where we have

$$
\begin{array}{lll}
\max _{\boldsymbol{P}_{c}^{v t}} \frac{f_{t, c}^{v t, l}\left(\boldsymbol{P}_{c}^{v t}\right)}{f_{p, c}^{v t}\left(\boldsymbol{P}_{c}^{v t}\right)} & \text { s.t. } & (22),(30) \\
\max _{\boldsymbol{P}_{c}^{c t}} \frac{f_{t, c}^{c t, l l}\left(\boldsymbol{P}_{c}^{c t}\right)}{f_{p, c}^{c t}\left(\boldsymbol{P}_{c}^{c t}\right)} & \text { s.t. } & (23),(31) \quad \forall c \in \mathcal{C}_{v t},
\end{array}
$$

Both (33) and (34) constitute fractional programming problems, which may be solved using Dinkelbach's method [38] by iteratively optimise the subtractive form of (33) and (34). More explicitly, for a particular $c \in \mathcal{C}_{v t}$, the procedures are

1) Introduce the parameter $t_{c, i}^{v t}$ with $i$ being the iteration index and initialise the parameter as $t_{c, 1}^{v t}=0$.

2) At each iteration $i$, solve the inner optimisation problem of the subtractive form of (33), namely

$$
\max _{\boldsymbol{P}_{c}^{v t}} f_{t, c}^{v t, l}\left(\boldsymbol{P}_{c}^{v t}\right)-t_{c, i}^{v t} f_{p, c}^{v t}\left(\boldsymbol{P}_{c}^{v t}\right) \quad \text { s.t. } \quad(22),(30) .
$$

Since the above inner optimisation problem is a concave maximisation problem, the classic dual-decomposition method yields a zero duality gap and hence achieves optimality. Owing to its popularity in solving convex problems, we refer readers for further details to [39].

3) Let $\boldsymbol{P}_{c}^{v t}$,* denote the optimal solutions found for the inner optimisation problem, if we have

$$
f_{t, c}^{v t, l}\left(\boldsymbol{P}_{c}^{v t, *}\right)-t_{c, i}^{v t} f_{p, c}^{v t}\left(\boldsymbol{P}_{c}^{v t, *}\right) \leq \zeta
$$

then the pre-defined convergence threshold $\zeta$ is satisfied, where $\boldsymbol{P}_{c}^{v t, *}$ constitutes the ultimate solution. Alternatively, if the maximum number of iterations $\zeta$ is reached, we output $\boldsymbol{P}_{c}^{v t, *}$ as the ultimate solution. Otherwise, we update the parameters according to

$$
t_{c, i+1}^{v t}=f_{t, c}^{v t, l}\left(\boldsymbol{P}_{c}^{v t, *}\right) / f_{p, c}^{v t}\left(\boldsymbol{P}_{c}^{v t, *}\right),
$$

and repeat Steps 2 and 3 .

The above procedures, referred to as the baseline algorithm ${ }^{7}$, can be also used for the energy efficiency maximisation of (34) for CT-aided cells and we do not duplicate it here.

3) Simplification: The baseline algorithm requires a number of iterations to converge, where within each iteration, the classic dual-decomposition method invoked, again, requires encapsulated iterations to converge. Hence, we propose an algorithm, which dispenses with the above sophistication. For any

\footnotetext{
${ }^{7}$ Other methods solving fractional programming, such as bisection and Charnes-Cooper method, are also interesting but they are beyond our scope.
}

particular VT-aided cell $c \in \mathcal{C}_{v t}$, we use the straightforward 596 equal power allocation strategy of

$$
P_{c, k}^{v t}=P_{c}^{v t} \quad \forall k \in \mathcal{K}_{c}
$$

To satisfy the constraint (30), we have

$$
\begin{aligned}
P_{c, k}^{v t} & \geq\left(2^{R / \kappa}-1\right)\left[\sigma^{2}+I_{c, k}\left(P_{\text {max }}^{\circ}\right)\right] 2 / \gamma^{2}=P_{c, k}^{v t, l}, \\
P_{c}^{v t} & \geq \max _{k \in \mathcal{K}_{c}} P_{c, k}^{v t, l}=P_{c}^{v t, l},
\end{aligned}
$$

while to satisfy the constraint (22), we have

$$
P_{c}^{v t} \leq 2 \pi\left(L P_{\text {max }}^{\circ}\right)^{2} / \sum_{k \in \mathcal{K}_{c}} g_{n, k}^{2}=P_{c}^{v t, u} .
$$

As a result, provided that $P_{c}^{v t, l} \leq P_{c}^{v t, u}$, we have

$$
P_{c}^{v t}=P_{c}^{v t, l}
$$

The solution $P_{c}^{v t}$ in (41) ensures that even the least privi- 601 leged user $k_{w}=\arg \max _{k \in \mathcal{K}_{c}}\left\{I_{c, k}\left(P_{\text {max }}^{\circ}\right)\right\}$ satisfies the per-user 602 throughput constraint of (30). However, setting $P_{c}^{v t}$ for other 603 users may 'over-satisfy' their per-user throughput constraint 604 of (30), which leads to an energy efficiency loss. Hence, we 605 improve the above equal power allocation strategy as

1) Let $\mathcal{K}_{c}^{f}$ be the set hosting those users with fixed power 607 allocation strategies, which is initialised as $\mathcal{K}_{c}^{f}=\left\{k_{w}\right\} .608$ Let $\mathcal{K}_{c}^{p}$ be the complement set of $\mathcal{K}_{c}^{f}$ hosting those users 609 with adjustable power allocation strategies. Finally, we 610 initialise $\boldsymbol{A}=\left\{P_{c, k \in \mathcal{K}_{c}}^{v t}=P_{c}^{v t}\right\}$.

2) For $k \in \mathcal{K}_{c}^{p}$, we reduce the power allocated from $P_{c, k}^{v t}=612$ $P_{c}^{v t}$ given in (41) to $P_{c, k}^{v t}=P_{c, k}^{v t, l}$ given in (38). This will 613 result in the adjusted power allocation strategy of

$$
\begin{aligned}
& \boldsymbol{A}_{k}=\left\{P_{c, k}^{v t}=P_{c, k}^{v t, l}, \boldsymbol{\Omega}_{k}\right\}, \\
& \boldsymbol{\Omega}_{k}=\left\{P_{c, j \in \mathcal{K}_{c}^{f}}^{v t}=P_{c, j}^{v t, l}, P_{c, \tilde{k} \in \mathcal{K}_{c}^{p}, \tilde{k} \neq k}^{v t}=P_{c}^{v t}\right\} .
\end{aligned}
$$

3) Repeating Step 2 for all users in $\mathcal{K}_{c}^{p}$ leads to $\left|\mathcal{K}_{c}^{p}\right|$ adjusted 615 power allocation strategies $\left\{\boldsymbol{A}_{k}, k \in \mathcal{K}_{c}^{p}\right\}$, where we find 616 the one gives the highest energy efficiency

$$
k_{p}=\arg \max _{k \in \mathcal{K}_{c}^{p}}\left\{f_{t, c}^{v t, l}\left(\boldsymbol{A}_{k}\right) / f_{p, c}^{v t}\left(\boldsymbol{A}_{k}\right)\right\} .
$$

4) If we have

$$
f_{t, c}^{v t, l}\left(\boldsymbol{A}_{k_{p}}\right) / f_{p, c}^{v t}\left(\boldsymbol{A}_{k_{p}}\right) \geq f_{t, c}^{v t, l}(\boldsymbol{A}) / f_{p, c}^{v t}(\boldsymbol{A}),
$$

then we include $k_{p}$ in $\mathcal{K}_{c}^{f}$ by excluding it from $\mathcal{K}_{c}^{p}$ and 619 set $\boldsymbol{A}=\boldsymbol{A}_{k_{p}}$. Then we repeat the procedure commencing 620 from Step 2. The adjustment stops, when (45) is not met 621 and we output $\boldsymbol{P}_{c}^{v t, *}=\boldsymbol{A}$.

For any particular CT-aided cell $c \in \mathcal{C}_{c t}$, we use the equal 623 power allocation strategy, namely

$$
P_{c, n}^{c t}=P_{c}^{c t} \quad \forall n \in \mathcal{N}_{c} .
$$

To satisfy the constraint (31), we have

$$
P_{c}^{c t} \geq \frac{\left(2^{R / \kappa}-1\right)\left[\sigma^{2}+I_{c, k_{0}}\left(P_{\max }\right)\right]}{\left(\gamma^{2} / 2\right) \sum_{n \in \mathcal{N}_{c}} h_{k_{0}, n}^{2}}=P_{c}^{c t, l},
$$


while to satisfy the constraint (23), we have

$$
P_{c}^{c t} \leq 2 \pi\left(L P_{\max }^{\circ}\right)^{2}=P_{c}^{c t, u} .
$$

As a result, provided that $P_{c}^{c t, l} \leq P_{c}^{c t, u}$, we have

$$
P_{c}^{c t}=P_{c}^{c t, l} \text {. }
$$

Let us now adjust the equal power allocation strategy by turning off the communications function for those APs exhibiting highattenuation channels, since blindly allocating power to it may lead to energy efficiency loss. More explicitly

1) Let $\mathcal{N}_{c}^{0}$ and $\mathcal{N}_{c}^{1}$ be the set hosting the specific APs whose communication functions are turned off and turned on, respectively, where we have $\mathcal{N}_{c}^{0}=\varnothing$ and $\mathcal{N}_{c}^{1}=\mathcal{N}_{c}$. Initialise $\boldsymbol{A}=\left\{P_{c, n \in \mathcal{N}_{c}}^{c t}=P_{c}^{c t}\right\}$.

2) Locate the weakest AP

$$
n_{w}=\arg \min _{n \in \mathcal{N}_{c}^{1}}\left\{h_{k_{0}, n}\right\}
$$

and include it in $\mathcal{N}_{c}^{0}$ by excluding it from $\mathcal{N}_{c}^{1}$.

3) Re-evaluate (47) and (48) using $\mathcal{N}_{c}^{1}$ instead of $\mathcal{N}_{c}$ in order to get $P_{c}^{c t, 1}$. Hence, we arrive at the new power allocation strategy of

$$
\boldsymbol{A}_{n_{w}}=\left\{P_{c, n \in \mathcal{N}_{c}^{0}}^{c t}=0, P_{c, n \in \mathcal{N}_{c}^{1}}^{c t}=P_{c}^{c t}{ }^{1}\right\}
$$

4) If we have

$$
f_{t, c}^{c t, l l}\left(\boldsymbol{A}_{n_{w}}\right) / f_{p, c}^{c t}\left(\boldsymbol{A}_{n_{w}}\right) \geq f_{t, c}^{c t, l l}(\boldsymbol{A}) / f_{p, c}^{c t}(\boldsymbol{A}),
$$

we set $\boldsymbol{A}=\boldsymbol{A}_{n_{w}}$ and repeat the above procedure, commencing from Step 2. The adjustments stop when (52) is not met and we output $\boldsymbol{P}_{c}^{c t, *}=\boldsymbol{A}$.

Remark II-C3: If no power adjustments are carried out, the algorithm proposed for the VT-aided or CT-aided cells only requires a one-off evaluation of (41) or (49), respectively. When adjustments are indeed required, the algorithm proposed requires at most $\left|\mathcal{K}_{c}\right|-1$ or $\left|\mathcal{N}_{c}\right|-1$ additional iterations for the VT-aided or CT-aided cells, respectively. Within each iteration, the dominant complexity component is only linearly increasing according to the order of $\mathcal{O}\left(\mathcal{K}_{c}^{p}\right)$ or $\mathcal{O}\left(\mathcal{N}_{c}^{1}\right)$ associated with evaluating (44) or (50) for the VT-aided or CT-aided cells, respectively. To further aid the reader's understanding, a flow chart is included in Fig. 2.

\section{NUMERICAL RESULTS}

Let us now provide simulation results for characterising the energy efficiency of the indoor VLC system relying on the amorphous structure. All our simulations are carried out for 100 random independent snapshots of the user distributions using the parameters included in Table I.

\section{A. Comparisons}

For fair comparisons, in Fig. 3, the same transmission strategy is employed and the same baseline optimisation algorithm is applied for all the cell formation strategies involved, where we have set the minimum per-user throughput constraint to $R=15 \mathrm{MBits} / \mathrm{s}$ for our optimisation algorithm.

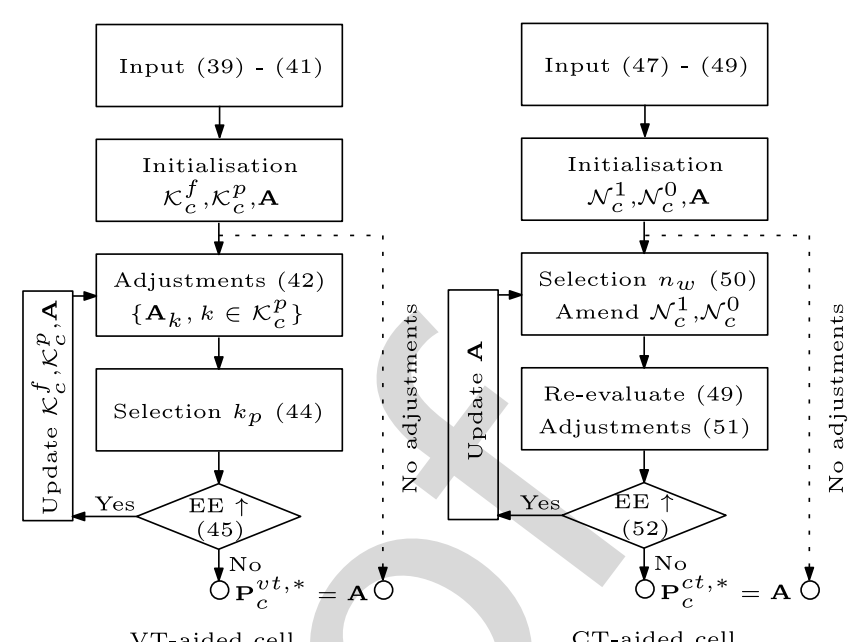

Fig. 2. Flowcharts of the proposed algorithm.

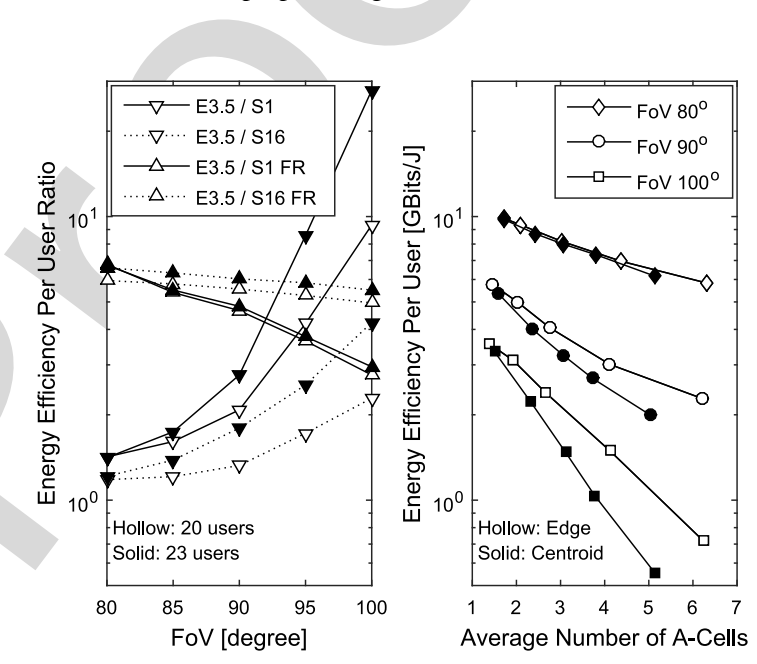

Fig. 3. Energy efficiency per user comparisons of various cell formation strategies (left) as well as between the edge and centroid-distance based A-Cells (right) using the parameters of Table I.

1) Edge-Distance-Based A-Cells vs. Conventional Cells: 668 The left subplot of Fig. 3 compares the energy efficiency 669 per user between the conventional cells and the edge-distance 670 based A-Cells having 20 users (hollow) and 25 users (solid). 671 Explicitly, 'E3.5' stands for the edge-distance based A-Cells 672 having $d_{0}=3.5 \mathrm{~m}$. This value of $d_{0}$ was specifically selected to 673 result in an average number of cells, which is similar to that of 674 the bench-marker scenario 'S16'. We also included the bench- 675 marker scenario ' $\mathrm{S} 1$ ' as introduced in Section II-A2. Since 676 similar trends may also be found for the centroid-distance based 677 A-Cells, we omit them for space economy. 678

It can be seen from the left subplot of Fig. 3 that, for both 679 20 users and 25 users, the edge-distance based A-Cells exhibit 680 a consistently higher energy efficiency per user than both the 681 conventional cell formations ' $\mathrm{S} 16$ ' and ' $\mathrm{S} 1$ ', where the relative 682 energy efficiency becomes significantly greater upon increasing 683 the FoVs when experiencing more interference. Quantitatively, 684 when having 20 users, the edge-distance based A-Cells are 685 capable of achieving over 4 times (nearly 10 times) energy effi- 686 ciency per user than that of the bench-marker scenario 'S1' 687 
at $\mathrm{FoV}$ of $95^{\circ}$ (of $100^{\circ}$ ). Similarly, when compared to the bench-marker scenario 'S16', the edge-distance based A-Cells are capable of roughly doubling the energy efficiency per user at both FoVs of $95^{\circ}$ and of $100^{\circ}$. These observations imply that the advocated edge-distance based A-Cells are capable of handling scenarios having more interference. This is true upon slightly increasing the number of users from 20 to 25 , where the edge-distance based A-Cells achieve overwhelmingly higher energy efficiency per user than both conventional cell formations 'S16' and 'S1'.

Furthermore, we included the classic scheme of frequency reuse having a factor of two in conjunction with both the conventional cell formations ' $\mathrm{S} 16$ ' and ' $\mathrm{S} 1$ '. It can be seen from the left subplot of Fig. 3 that the edge-distance based A-Cells exhibit a consistently higher energy efficiency per user than both the conventional cell formations relying on frequency reuse for all FoV settings and for both user settings. For all FoVs, as expected, the higher the number of users, the higher the energy efficiency per user of the edge-distance based A-Cells becomes in comparison to both conventional cell formations. Upon increasing the FoVs, the energy efficiency per user achieved by the edge-distance based A-Cells is reduced for both conventional cell formations as well as for both user settings, exhibiting a steeper reduction for the conventional cell formation ' $\mathrm{S} 1$ '. This is because, the higher the FoV, the more interference is encountered, hence frequency reuse becomes more beneficial. Despite this reduction, the energy efficiency per user achieved by the edge-distance based A-Cells remains at least three times higher when compared to that of the conventional cell formation ' $\mathrm{S} 1$ ' employing frequency reuse and supporting 20 users. To sum up, the edge-distance based A-Cells are significantly more energy efficient than both the conventional cell formations ' $\mathrm{S} 16$ ' and ' $\mathrm{S} 1$ ' operating with or without frequency reuse.

2) Edge-Distance vs. Centroid-Distance-Based A-Cells: Owing to the flexibility of the distance based A-Cells, we can appropriately configure them to provide a fair comparison. The right subplot of Fig. 3 compares the energy efficiency per user between the edge-distance (hollow) and the centroid-distance (solid) based A-Cells with the average number of A-Cells spanning from 1 to 6 . For the edge-distance based A-Cells, we evaluate $d_{0}=[3,3.5,4,4.5,5]$, while for the centroid-distance based A-Cells, we evaluate $d_{0}=[5,6,7,8,9]$. Note that the higher the value of $d_{0}$, the smaller the average number of resultant A-Cells. It can be seen from the right subplot of Fig. 3 that the edge-distance based A-Cells exhibit a consistently higher energy efficiency per user than that of the centroid-distance based A-Cells for all resultant average number of A-Cells and for all FoVs, which shows the superiority of the edge-distance based A-Cells. Indeed, we observe that the superiority of the edge-distance based A-Cells is more prominent at higher FoVs for all resultant average number of A-Cells, while they become very similar at FoV of $80^{\circ}$. Finally, at FoVs of $90^{\circ}$ and of $100^{\circ}$, we observe that the superiority of edge-distance based A-Cells is greater when having a larger number of resultant ACells, whilst there are marginal differences when the number of A-Cells is small.
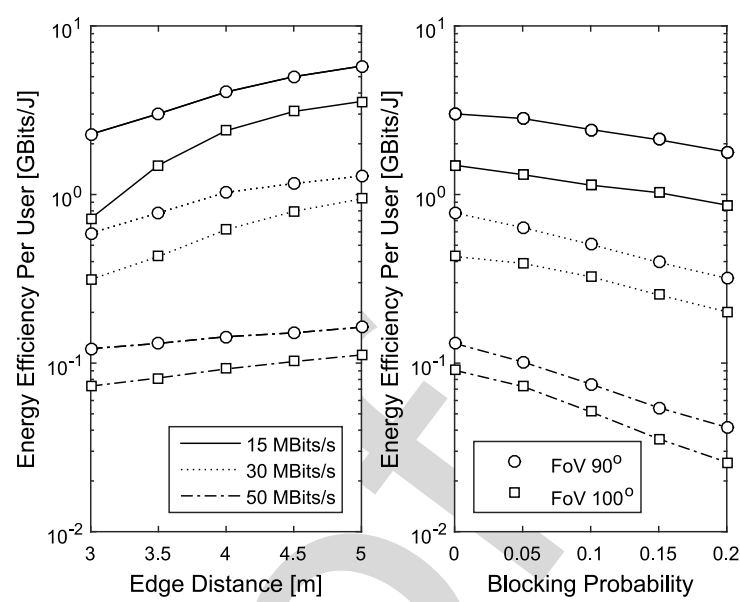

Fig. 4. Energy efficiency per user for edge-distance based A-Cells as a function of the predefined edge-distance threshold (left) and of the LoS and reflectedpath blocking probability (right) using the parameters of Table I.

\section{B. Details}

We now provide detailed observations regarding the edge- 746 distance based A-Cells. In these investigations, we use our 747 baseline algorithm for optimisation. Since similar trends may 748 also be found for the centroid-distance based A-Cells, we omit 749 them for space economy.

1) Effect of Edge Distance: The left subplot of Fig. 4 shows 751 the energy efficiency per user for edge-distance based A-Cells 752 as a function of the predefined edge-distance threshold. It can 753 be seen from the left subplot of Fig. 4 that as expected, guaran- 754 teeing a higher per-user throughput incurs an energy efficiency 755 loss compared to requiring a lower per-user throughput for all 756 predefined edge-distance threshold settings and for both FoVs, 757 demonstrating that any throughput improvement requires extra 758 power to be invested ${ }^{8}$. Furthermore, for all throughput con- 759 straints and for all predefined edge-distance threshold settings, 760 having a higher FoV results in a consistently lower energy 761 efficiency per user than that of a lower FoV, since more inter- 762 ference is encountered and more APs are involved when having 763 a higher FoV. Finally, we observe that the higher the edge- 764 distance threshold, the higher the energy efficiency per user 765 becomes for all throughput constraints and for both FoVs. 766 However, at this stage, we are reluctant to claim the supe- 767 riority of setting towards higher edge-distance threshold for 768 the reasons discussed as follows. In principle, setting a lower 769 edge-distance threshold results into several decoupled A-Cells, 770 whilst having a higher edge-distance threshold results into a 771 few large A-Cells. In both settings, the total number of par- 772 ticipated APs remains similar under a given FoV. Having a 773 few large A-Cells creates a large-dimensional multi-user MISO 774 system. Hence, it is capable of more easily satisfying a given 775 throughput constraint than forming several decoupled A-Cells. 776 However, having a large-dimensional multi-user MISO system 777 will potentially incur additional signal processing costs, such as 778

\footnotetext{
${ }^{8}$ Different modulations incur different levels of energy investments. For example, Color Shift Keying (CSK) relies on the LEDs' color mapping capability, instead of higher DC power, for achieving an increased data rate.
} 
the inversion of a large matrix at the distributed APs as required by the ZF based TPC. Also, sharing data amongst APs of large A-Cells may require more capable back-haul. Hence, network power consumption by additionally considering the signal processing costs and back-haul power consumption is required to determine the most appropriate edge-distance threshold.

2) Effect of Blocking: The right subplot of Fig. 4 shows the energy efficiency per user for edge-distance based A-Cells associated with $d_{0}=3.5 \mathrm{~m}$ as a function of the $\operatorname{LoS}$ and reflected-path blocking probability. It can be seen from the right subplot of Fig. 4 that as expected, the higher the blocking probability, the lower the energy efficiency per user becomes for all throughput constraints and for both FoVs. Furthermore, for all blocking probabilities and for both FoVs, the energy efficiency per user is higher for a lower throughput constraint than for a higher throughput constraint. We observe also that, the slope of the energy efficiency reduction per user is higher for a higher throughput constraint for both FoVs. Finally, for all throughput constraints and for all blocking probabilities, having a higher FoV results in a consistently lower energy efficiency per user than that of a lower FoV, since more interference is encountered and more APs are involved when having a higher FoV. Note that, the blocking model considered here is independent of the FoVs, while we will consider a more realistic FoV-related blocking in the future.

3) Effect of Imperfect Channel Knowledge: The left of Fig. 5 shows the energy efficiency per user for edge-distance based A-Cells associated with $d_{0}=3.5 \mathrm{~m}$ as a function of the error variance of imperfect channel knowledge at the distributed APs used for VT or CT. We assumed Gaussian distributed errors and as expected, the achievable energy efficiency per user degrades upon increasing the error variance for all throughput constraints and for both FoVs. Furthermore, for all error variances and for both FoVs, the energy efficiency per user is higher for a lower throughput constraint than for a higher throughput constraint. Furthermore, for all throughput constraints and for all error variances, having a higher FoV results in a consistently lower energy efficiency per user than that of a lower FoV. These investigations imply the importance of having an accurate channel knowledge, where a modest reduction may be observed in the left of Fig. 5 at an error variance of 0.2 for all throughput constraints and for both FoVs. Hence, a classic trade-off arises between reducing the error variance and investing extra cost, which will be set aside for our future work.

4) Effect of User Density: The right subplot of Fig. 5 shows the energy efficiency per user for edge-distance based A-Cells associated with $d_{0}=3.5 \mathrm{~m}$ as a function of user density. It can be seen from the right subplot of Fig. 5 that as expected for all throughput constraints and for both FoVs, the higher the number of users, the lower the energy efficiency per user becomes, since the interference becomes more pervasive and more number of APs are involved. Importantly, the most substantial drop appears upon increasing the number of users from 10 to 20, followed by a less dramatic energy efficiency erosion per user beyond 20 users. Furthermore, for all user density settings and for both FoVs, the energy efficiency per user is higher for a lower throughput constraint than for a higher throughput constraint. Finally, for all throughput constraints and for all user

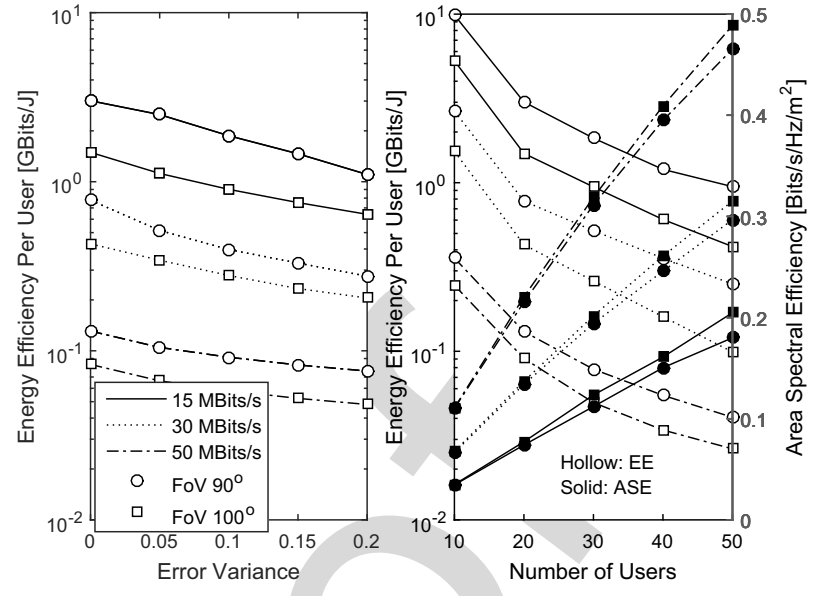

Fig. 5. Energy efficiency per user for edge-distance based A-Cells associated with $d_{0}=3.5 \mathrm{~m}$ as a function of error variance (left) and energy efficiency per user versus area spectral efficiency trade-offs (right) as a function of user density using the parameters of Table I.

density settings, having a higher FoV results in a consistently 837 lower energy efficiency per user than that of a lower FoV, since 838 more interference is encountered and more APs are involved 839 when having a higher FoV.

5) Trade-Offs: The right subplot of Fig. 5 also shows the 841 Area Spectral Efficiency (ASE) for edge-distance based A- 842 Cells associated with $d_{0}=3.5 \mathrm{~m}$ as a function of user density. It 843 can be seen from the right subplot of Fig. 5 that as expected, for 844 all user density settings and for both FoVs, the ASE is higher 845 for a higher throughput constraint than for a lower throughput 846 constraint. Furthermore, for all throughput constraints and for 847 both FoVs, the higher the number of users, the higher the ASE 848 becomes. This is because the aggregated throughput is higher 849 for a higher number of users. However, the ASE improvement 850 seen in the right subplot of Fig. 5 is achieved at the cost of 851 sacrificing the energy efficiency per user. Achieving a higher 852 ASE at the cost of reducing the energy efficiency contradicts 853 to our original design objective. Similarly, for all throughput 854 constraints and for all user density settings, having a higher 855 FoV results in a slightly higher energy efficiency per user than 856 that of a lower FoV, again at the cost of sacrificing the energy 857 efficiency. Finally, we note that the ASE recorded in the right 858 subplot of Fig. 5 is not the maximum achievable ASE, since it 859 was evaluated under the specific constraint of the power alloca- 860 tion strategy obtained when using the energy efficiency as our 861 design objective.

\section{Algorithms}

We now discuss the performance of our proposed algorithm, 864 where we set the minimum per-user throughput constraint to 865 $R=15 \mathrm{MBits} / \mathrm{s}$ and FoV of $90^{\circ}$ for our investigations. 866

1) Comparison: The left subplot of Fig. 6 shows the energy 867 efficiency per user for edge-distance based A-Cells as a func- 868 tion of the predefined edge-distance threshold, when using 869 both the baseline algorithm and the proposed algorithm with 870 and without adjustments, as detailed in Section II-C3. For our 871 proposed algorithm relying on adjustments, we set $\left|\mathcal{K}_{c}\right|-1872$ and $\left|\mathcal{N}_{c}\right|-1$ as the maximum number of iterations for our 873 

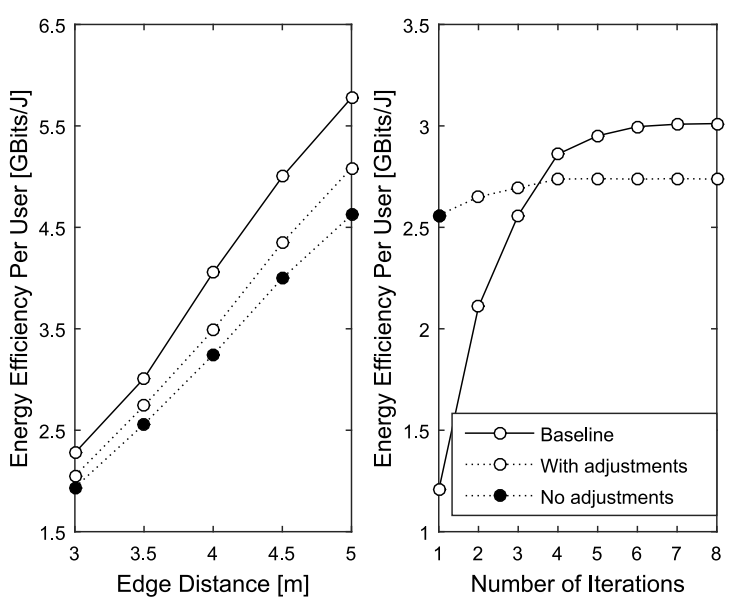

Fig. 6. Energy efficiency per user for edge-distance based A-Cells as a function of the predefined edge-distance threshold (left) and of the number of iterations (right) when using both the baseline algorithm and the proposed algorithm with and without adjustments and using the parameters of Table I.

VT and CT-aided A-Cells, respectively. It can be seen from the left subplot of Fig. 6 that the proposed algorithm operating without adjustments is capable of achieving much of the energy efficiency per user obtained by the baseline algorithm. When relying on additional adjustments, the proposed algorithm achieves a higher energy efficiency per user than that of its non-adjusted counterparts. In particular, it performs quite similarly to the baseline algorithm, when the predefined edge-distance threshold is small.

2) Convergence: The right subplot of Fig. 6 shows the convergence of both the baseline and of the proposed algorithm for edge-distance based A-Cells associated with $d_{0}=3.5 \mathrm{~m}$. It can be seen that the baseline algorithm converges within 6 iterations with further embedded iterations owing to the usage of the dual-decomposition method. For our proposed algorithm, 3 iterations are typically sufficient to approach convergence dispensing with the dual-decomposition method. Despite the ultimate sub-optimality, the intermediate energy efficiency per user achieved by our proposed algorithm is much higher than that of the baseline algorithm. This becomes especially prominent, when we only use a single iteration, which is marked by the solid circle. In the right subplot of Fig. 6, quantitatively, the achievable energy efficiency per user of our proposed algorithm after 3 iterations accounts for slightly more than $90 \%$ that of its ultimate counterpart constituted by our baseline algorithm.

\section{CONCLUSION}

In this paper, we discussed the hitherto-unexplored amorphous structure for constructing energy efficient VLC systems. This problem was approached by the joint design of three inter-linked aspects under the critical consideration of optical constraints. Our numerical results demonstrated that the amorphous structure proposed is beneficial in VLC system design, since it results in a higher energy efficiency than that of the conventional structure. Furthermore, our proposed algorithm is capable of performing close to the baseline algorithm, making it an attractive design alternative. Finally, our proposed amorphous structure constitutes a promising LiFi solution, providing services in various indoor applications, including museums, offices and hospitals. These observations stimulate a range of 912 further research topics, such as the energy efficient VLC system 913 design taking into account the clipping-distortion of DCO- 914 OFDM, the co-design of energy efficient communication and 915 illumination, the network power consumption of VLC system, 916 the comparison to other OOFDM schemes [37]-[41], the inves- 917 tigation of non-linear VT, and finally the robust design under 918 channel estimation uncertainties, etc.

\section{REFERENCES}

[1] G. Li et al., "Energy-efficient wireless communications: Tutorial, survey, 921 and open issues," IEEE Wireless Commun., vol. 18, no. 6, pp. 28-35, Dec. 922 2011.

[2] G. Miao, N. Himayat, and G. Li, "Energy-efficient link adaptation in 924 frequency-selective channels," IEEE Trans. Commun., vol. 58, no. 2, 925 pp. 545-554, Feb. 2010.

[3] D. Ng, E. Lo, and R. Schober, "Energy-efficient resource allocation in 927 OFDMA systems with large numbers of base station antennas," IEEE 928 Trans. Wireless Commun., vol. 11, no. 9, pp. 3292-3304, Sep. 2012.

[4] S. He, Y. Huang, L. Yang, and B. Ottersten, "Coordinated multicell mul- 930 tiuser precoding for maximizing weighted sum energy efficiency," IEEE 931 Trans. Signal Process., vol. 62, no. 3, pp. 741-751, Feb. 2014.

[5] C. He, B. Sheng, P. Zhu, X. You, and G. Li, "Energy- and spectral- 933 efficiency tradeoff for distributed antenna systems with proportional 934 fairness," IEEE J. Sel. Areas Commun., vol. 31, no. 5, pp. 894-902, May 935 2013.

[6] L. B. Le, D. Niyato, E. Hossain, D. I. Kim, and D. T. Hoang, "QoS-aware 937 and energy-efficient resource management in OFDMA femtocells," IEEE 938 Trans. Wireless Commun., vol. 12, no. 1, pp. 180-194, Jan. 2013.

[7] S. Rangan, T. Rappaport, and E. Erkip, "Millimeter-wave cellular wire- 940 less networks: Potentials and challenges," Proc. IEEE, vol. 102, no. 3, 941 pp. 366-385, Mar. 2014.

[8] L. Hanzo, H. Haas, S. Imre, D. O'Brien, M. Rupp, and L. Gyongyosi, 943 "Wireless myths, realities, and futures: From $3 \mathrm{G} / 4 \mathrm{G}$ to optical and 944 quantum wireless," Proc. IEEE, vol. 100, no. Special Centennial Issue, 945 pp. 1853-1888, May 2012.

[9] E. Bjornson, L. Sanguinetti, J. Hoydis, and M. Debbah, “Optimal design 947 of energy-efficient multi-user MIMO systems: Is massive MIMO the 948 answer?," IEEE Trans. Wireless Commun., vol. 14, no. 6, pp. 3059-3075, 949 Jun. 2015 .

[10] R. Zhang, L.-L. Yang, and L. Hanzo, "Energy pattern aided simultaneous 951 wireless information and power transfer," IEEE J. Sel. Areas Commun., 952 vol. 33, no. 8, pp. 1492-1504, Aug. 2015.

[11] R. Zhang, R. Maunder, and L. Hanzo, "Wireless information and 954 power transfer: From scientific hypothesis to engineering practice," IEEE 955 Commun. Mag., vol. 53, no. 8, pp. 99-105, Aug. 2015.

[12] R. Zhang, J. Wang, Z. Wang, Z. Xu, C. Zhao, and L. Hanzo, "Visible 957 light communications in heterogeneous networks: Pave the way for user- 958 centric design," IEEE Wireless Commun., vol. 22, no. 2, pp. 8-16, Apr. 959 2015.

[13] T. Komine and M. Nakagawa, "Fundamental analysis for visible- 961 light communication system using LED lights," IEEE Trans. Consum. 962 Electron., vol. 50, no. 1, pp. 100-107, Feb. 2004.

[14] J. Grubor, S. Randel, K.-D. Langer, and J. Walewski, "Broadband information broadcasting using LED-based interior lighting," J. Lightw. Technol., vol. 26, no. 24, pp. 3883-3892, Dec. 2008.

[15] R. Zhang and L. Hanzo, "Multi-layer modulation for intensity-modulated 967 direct-detection optical OFDM," J. Opt. Commun. Netw., vol. 5, no. 12, 968 pp. 1402-1412, Dec. 2013.

[16] J. Armstrong, "OFDM for optical communications," J. Lightw. Technol., 970 vol. 27, no. 3, pp. 189-204, Feb. 2009.

[17] A. Azhar, T Tran, and D. O'Brien "A Gigabit/s indoor wireless transmis- 972 sion using MIMO-OFDM visible-light communications," IEEE Photon. 973 Technol. Lett., vol. 25, no. 2, pp. 171-174, Jan. 2013.

[18] D. Tsonev et al., "A 3-Gb/s single-LED OFDM-based wireless VLC link 975 using a gallium nitride $\mu$ LED," IEEE Photon. Technol. Lett., vol. 26, 976 no. 7, pp. 637-640, Apr. 2014.

[19] H. Burchardt, N. Serafimovski, D. Tsonev, S. Videv, and H. Haas, "VLC: 978 Beyond point-to-point communication," IEEE Commun. Mag., vol. 52, 979 no. 7, pp. 98-105, Jul. 2014.

[20] B. Ghimire and H. Haas, "Self-organising interference coordination in optical wireless networks," EURASIP J. Wireless Commun. Netw., vol. 2012 , no. $1,2012$.

\section{.}


[21] D. Bykhovsky and S. Arnon, "Multiple access resource allocation in visible light communication systems," J. Lightw. Technol., vol. 32, no. 8, pp. 1594-1600, Apr. 2014.

[22] M. Biagi, S. Pergoloni, and A. M. Vegni, "LAST: A framework to localize, access, schedule, and transmit in indoor VLC systems," J. Lightw. Technol., vol. 33, no. 9, pp. 1872-1887, May 2015.

[23] F. Jin, R. Zhang, and L. Hanzo, "Resource allocation under delayguarantee constraints for heterogeneous visible-light and RF femtocell," IEEE Trans. Wireless Commun., vol. 14, no. 2, pp. 1020-1034, Feb. 2015

[24] X. Li, R. Zhang, and L. Hanzo, "Cooperative load balancing in hybrid visible light communications and WiFi," IEEE Trans. Commun., vol. 63, no. 4, pp. 1319-1329, Apr. 2015.

[25] K. Lee and H. Park, "Modulations for visible light communications with dimming control," IEEE Photon. Technol. Lett., vol. 23, no. 16, pp. 1136 1138, Aug. 2011.

[26] I. Din and H. Kim, "Energy-efficient brightness control and data transmission for visible light communication," IEEE Photon. Technol. Lett., vol. 26, no. 8, pp. 781-784, Apr. 2014

[27] S. Kim and S.-Y. Jung, "Modified Reed Muller coding scheme made from the bent function for dimmable visible light communications," IEEE Photon. Technol. Lett., vol. 25, no. 1, pp. 11-13, Jan. 2013.

[28] Y. Suh, C.-H. Ahn, and J. K. Kwon, "Dual-codeword allocation scheme for dimmable visible light communications," IEEE Photon. Technol. Lett., vol. 25, no. 13, pp. 1274-1277, Jul. 2013.

[29] R. Razavi and H. Claussen, "Urban small cell deployments: Impact on the network energy consumption," in Proc. IEEE Wireless Commun. Netw. Conf. Workshops, Apr. 2012, pp. 47-52.

[30] S. Dimitrov, S. Sinanovic, and H. Haas, "Clipping noise in OFDM-based optical wireless communication systems," IEEE Trans. Commun., vol. 60, no. 4, pp. 1072-1081, Apr. 2012.

[31] A. Farid and S. Hranilovic, "Capacity bounds for wireless optical intensity channels with gaussian noise," IEEE Trans. Inf. Theory, vol. 56, no. 12, pp. 6066-6077, Dec. 2010.

[32] X. Li, R. Mardling, and J. Armstrong, "Channel capacity of IM/DD optical communication systems and of ACO-OFDM," in Proc. IEEE Int. Conf. Commun., Jun. 2007, pp. 2128-2133.

[33] S. Dimitrov and H. Haas, "Information rate of OFDM-based optical wireless communication systems with nonlinear distortion," J. Lightw. Technol., vol. 31, no. 6, pp. 918-929, Mar. 2013.

[34] M. Costa, "Writing on dirty paper," IEEE Trans. Inf. Theory, vol. 29, no. 3, pp. 439-441, May 1983.

[35] J. Lee and N. Jindal, "High SNR analysis for MIMO broadcast channels: Dirty paper coding versus linear precoding," IEEE Trans. Inf. Theory, vol. 53, no. 12, pp. 4787-4792, Dec. 2007.

[36] R. Zhang and L. Hanzo, "Cooperative downlink multicell preprocessing relying on reduced-rate back-haul data exchange," IEEE Trans. Veh. Technol., vol. 60, no. 2, pp. 539-545, Feb. 2011.

[37] Z. Wang, Q. Wang, S. Chen, and L. Hanzo, "An adaptive scaling and biasing scheme for OFDM-based visible light communication systems," Opt. Exp., vol. 22, no. 10, pp. 12 707-12 715, May 2014.

[38] W. Dinkelbach, "On nonlinear fractional programming," Manage. Sci., vol. 13, pp. 492-498, Mar. 1967.

[39] S. Boyd and L. Vandenberghe, Convex Optimization. Cambridge, U.K.: Cambridge Univ. Press, 2004.

[40] L. Chen, B. Krongold, and J. Evans, "Successive decoding of antiperiodic OFDM signals in IM/DD optical channel," in Proc. IEEE Int. Conf. Commun., May 2010, pp. 1-6.

[41] Q. Gao, C. Gong, S. Li, and Z. Xu, "DC-informative modulation for visible light communications under lighting constraints," IEEE Wireless Commun., vol. 22, no. 2, pp. 54-60, Apr. 2015.

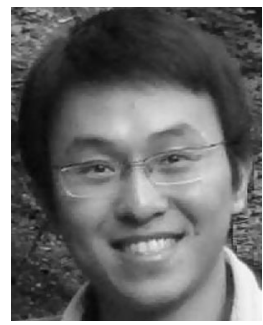

Rong Zhang (M'09) received the Ph.D. degree in wireless communications from University of Southampton (UoS), Southampton, U.K., in 2009. He is an Assistant Professor with Southampton Wireless Group, School of Electronics and Computer Science, UoS. He was with UoS, as a Research Assistant during that period with Mobile Virtual Centre of Excellence, one of UK's largest industrial-academic partnership in ICT. During his postdoctoral period at ECS, he contributed as the UoS Lead Researcher on a number of international projects. After that, he took his industrial consulting leave for Huawei EU R\&D as a System Algorithms Expert. He is also a Guest Researcher of the Centre in Next Generation Computational Modelling, UoS. He has a total of 70+ IEEE/OSA publications, including $40+$ journals (20+ of which as first author). He regularly serves as a Reviewer for the IEEE/OSA journals and has been several times a TPC
member/Invited Session Chair of major conferences. He is a member of the 1060 IET and is the recipient of joint funding from MVCE and EPSRC as well 1061 as the recipient of Worldwide University Network Grant. Owing to his out- 1062 standing academic achievements, he was the recipient of the Prestigious Dean's 1063 Publication Award.

1064

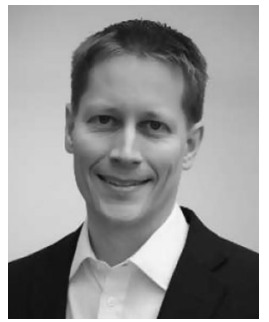

Holger Claussen (S'02-M'04-SM'10) received the 1065 Ph.D. degree in signal processing for digital com- 1066 munications from the University of Edinburgh, 1067 Edinburgh, U.K., in 2004. He is Leader of Small Cells 1068 Research, Bell Labs, Alcatel-Lucent. In this role, he 1069 and his team are innovating in all areas related to 1070 future evolution, deployment, and operation of small 1071 cell networks to enable exponential growth in mobile 1072 data traffic. His research in this domain has been com- 1073 mercialized in Alcatel-Lucent's Small Cell product 1074 portfolio and continues to have significant impact. 1075 Prior to this, he was the Head of the Autonomous Networks and Systems 1076 Research Department, Bell Labs Ireland, where he directed research in the area 1077 of self-managing networks to enable the first large scale femtocell deployments 1078 from 2009 onwards. He joined Bell Labs in 2004. His research interests include 1079 network optimization, cellular architectures, and improving energy efficiency 1080 of networks. He is the author of more than 90 publications and 110 filed patent 1081 applications. He is a Fellow of the World Technology Network and member 1082 of the IET. He was the recipient of the 2014 World Technology Award in the 1083 individual category Communications Technologies for innovative work of the 1084 greatest likely long-term significance.

1085

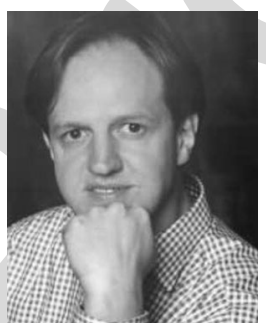

Harald Haas (S'98-M'03) holds the Chair for 1086 Mobile Communications, School of Engineering, 1087 and is the Director of the Li-Fi Research and 1088 Development Centre. He has been working in wire- 1089 less communications for 20 years and held several 1090 posts in industry. He was an invited speaker at TED 1091 Global in 2011, where he demonstrated and coined 1092 $\mathrm{LiFi}$. Li-Fi was listed among the 50 best inventions 1093 in TIME Magazine 2011. Moreover, his work has 1094 been covered in other international media such as the 1095 New York Times, BBC, MSNBC, CNN International, 1096 Wired U.K., and many more. He is an Initiator, a Co-Founder, and the Chief 1097 Scientific Officer (CSO) of pureLiFi Ltd. He holds 31 patents and has more 1098 than 30 pending patent applications. He has authored 300 conference and jour- 1099 nal papers including a paper in Science Magazine. He published two textbooks 1100 with Cambridge University Press. His h-index is 43 (Google). He is CI of pro- 1101 gramme grant TOUCAN (EP/L020009/1), and CI of SERAN (EP/L026147/1). 1102 He currently holds an EPSRC Established Career Fellowship (EP/K008757/1). 1103 In 2014, he was selected as one of ten EPSRC RISE Campaign Leaders. In 1104 2015, he was corecipient of three best paper awards including the IEEE Jack 1105 Neubauer Memorial Award.

1106

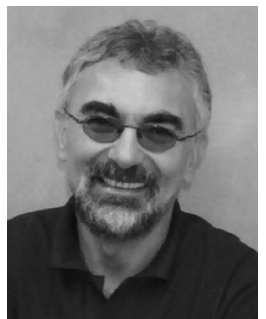

Lajos Hanzo (M'91-SM'92-F'04) received the 1107 degree in electronics, in 1976, the doctorate degree, 1108 in 1983, and the D.Sc. degree. In 2009, he was 1109 awarded the honorary doctorate "Doctor Honoris 1110 Causa" by the Technical University of Budapest. 1111 During his 38-year career in telecommunications, 1112 he has held various research and academic posts in 1113 Hungary, Germany and the U.K. Since 1986, he has 1114 been with the School of Electronics and Computer 1115 Science, University of Southampton, Southampton, 1116 U.K., where he holds the Chair in telecommunica- 1117 tions. He has successfully supervised 80+ Ph.D. students, coauthored 20 John 1118 Wiley/IEEE Press books on mobile radio communications totalling in excess of 1119 10000 pages, published $1400+$ research entries at IEEE Xplore, acted both as 1120 a TPC and the General Chair of the IEEE conferences, presented keynote lec- 1121 tures, and has been awarded a number of distinctions. Currently, he is directing 1122 a 100 -strong academic research team, working on a range of research projects 1123 in the field of wireless multimedia communications sponsored by industry, 1124 the Engineering and Physical Sciences Research Council (EPSRC) U.K., the 1125 European Research Council's Advanced Fellow Grant, and the Royal Society's 1126 Wolfson Research Merit Award. He is an enthusiastic supporter of industrial 1127 and academic liaison and offers a range of industrial courses. He is also a 1128 Governor of the IEEE VTS. From 2008 to 2012, he was the Editor-in-Chief 1129 of the IEEE Press and a Chaired Professor also at Tsinghua University, Beijing, 1130 China. His research is funded by the European Research Council's Senior 1131 Research Fellow Grant. He has $20000+$ citations. He is a Fellow of REng, IET, 1132 and EURASIP. 


\section{QUERIES}

Q1: Please be advised that per instructions from the Communications Society this proof was formatted in Times Roman font and therefore some of the fonts will appear different from the fonts in your originally submitted manuscript. For instance, the math calligraphy font may appear different due to usage of the usepackage[mathcal]euscript. We are no longer permitted to use Computer Modern fonts.

Q2: Note that if you require corrections/changes to tables or figures, you must supply the revised files, as these items are not edited for you.

Q3: Please provide page range for Ref. [20]. 